\title{
Strain-Programmable Patch for Diabetic Wound Healing
}

Georgios Theocharidis ${ }^{1 *}$, Hyunwoo Yuk ${ }^{2 *}$, Heejung Roh $^{2 *}$, Liu Wang ${ }^{2,3^{*}}$, Ikram Mezghani ${ }^{1}$, Jingjing $\mathrm{Wu}^{2,3}$, Antonios Kafanas ${ }^{4}$, Lihong Chen ${ }^{1}$, Chuan Fei Guo ${ }^{3}$, Navin Jayaswal ${ }^{1}$, XanthiLeda Katopodi ${ }^{5}$, Christoph S. Nabzdyk ${ }^{6}$, Ioannis S. Vlachos ${ }^{5,7}$, Aristidis Veves ${ }^{1 \dagger+}$, Xuanhe Zhao $2,8+*$

${ }^{1}$ Joslin-Beth Israel Deaconess Foot Center and The Rongxiang Xu, MD, Center for Regenerative Therapeutics, Beth Israel Deaconess Medical Center, Harvard Medical School, Boston, MA, USA

${ }^{2}$ Department of Mechanical Engineering, Massachusetts Institute of Technology, Cambridge, MA, USA

${ }^{3}$ Department of Materials Science and Engineering, Southern University of Science and Technology, Shenzhen, China

${ }^{4}$ Lincoln County Hospital, Northern Lincolnshire and Goole NHS Foundation Trust, UK

${ }^{5}$ Cancer Research Institute, HMS Initiative for RNA Medicine, Department of Pathology, Beth Israel Deaconess Medical Center, Harvard Medical School, Boston, MA, USA

${ }^{6}$ Department of Anesthesiology and Perioperative Medicine, Mayo Clinic, Rochester, MN, USA

${ }^{7}$ Broad Institute of MIT and Harvard, Cambridge, MA, USA

${ }^{8}$ Department of Civil and Environmental Engineering, Massachusetts Institute of Technology, Cambridge, MA, USA

* These authors contributed equally to this work.

$\dagger$ These authors jointly supervised this work.

† Corresponding author. Email: hyunwoo@mit.edu (H.Y.); aveves@ bidmc.harvard.edu (A.V.); zhaox@mit.edu (X.Z.)

One Sentence Summary: A strain-programmable bioadhesive patch is developed for accelerated closure and healing of wounds in diabetic mice and human skin. 


\begin{abstract}
Chronic wounds with impaired healing capability such as diabetic foot ulcers (DFU) are devastating complications in diabetic patients, inflicting rapidly growing clinical and economic burdens in aging societies. Despite recent advances in therapeutic approaches, limited benefits of the existing solutions highlight the critical need for novel therapeutic solutions for diabetic wound healing. Here we propose a strain-programmable patch capable of rapid robust adhesion on and programmable mechanical contraction of wet wounded tissues over days to offer a new therapeutic platform for diabetic wounds. The strain-programmable patch, consisting of a dried bioadhesive layer and a pre-stretched elastomer backing, implements a hydration-based shape-memory mechanism to achieve both uniaxial and biaxial contractions and stress remodeling of wet wounds in a programmable manner. We develop theoretical and numerical models to rationally guide the strain-programming and mechanical modulation of wounds. In vivo rodent and ex vivo human skin culture models validate the programmability and efficacy of the proposed platform and identify mechanisms of action for accelerated diabetic wound healing.
\end{abstract}




\section{INTRODUCTION}

Impaired wound healing capability and consequent chronic wounds such as diabetic foot ulcers (DFU) are one of the major and rapid growing complications in diabetic patients with over 750,000 new DFU each year $(1,2)$. Chronic DFU inflict significant clinical and economic burdens including 70,000 lower extremity amputations, a dramatic reduction in life quality (2) and associated costs over 11 billion dollars annually in the U.S. alone (3). Although various therapeutic strategies, such as bioengineered skin $(4,5)$ and growth factor-based treatments $(6)$ have been introduced in clinical practice in the last few decades, their benefits are rather limited as more than $50 \%$ of treated DFU patients fail to respond $(7,8)$. The rapidly rising number of diabetic patients worldwide and the lack of effective treatment highlight the critical importance of developing new therapeutic solutions for diabetic wound healing.

Mechanical modulation of wounded or scarred skin has been a promising strategy to repair and remodel the skin in both animal models and human clinical trials (9-17). In addition, animal studies have indicated that the reduced contractibility of diabetic wounds compared to non-diabetic wounds is one of the sources of impaired diabetic wound healing (7, 15, 18-20). Therefore, mechanical modulation such as inducing contraction of diabetic wounds can be an attractive approach to accelerate diabetic wound healing. However, the potential therapeutic benefits of the mechanical modulation approach have not been well investigated for diabetic wounds such as DFU due to several technical limitations. Existing wound dressings and bandages for mechanical reinforcement or stimulation lack the capabilities to form rapid and robust adhesion on wet wounded skin over the long term (e.g., days) or to precisely program the mechanical contraction for wounds, limiting their use only for passive coverage, ineffective and uncontrolled contraction, and/or fully closed wounds $(13,21-23)$. To the best of our knowledge, there exists no method that can exert precisely-controlled and long-term contraction on wet wounded skin, leaving this potential therapeutic strategy untapped for diabetic wounds.

In this work, we report a strain-programmable patch capable of programmable and consistent mechanical contraction of wet wounded tissues over days as a promising therapeutic platform for accelerated healing of diabetic wounds. The strain-programmable patch synergistically combines a dry-crosslinking mechanism and a hydration-based shape-memory mechanism to simultaneously achieve robust, long-lasting, and on-demand detachable adhesion on wet wounded tissues and precisely-controlled mechanical modulation of the wounds, respectively. The strain-programmable patch takes the form of a thin flexible film consisting of a dry bioadhesive in the glassy state (high Young's modulus) and a pre-stretched non-adhesive elastomer backing in the rubbery state (low Young's modulus) with the programmed strain (Fig. 1A). The programmed strain in the patch is maintained due to the constraint of the dry bioadhesive. Once hydrated by the native physiological fluids and/or moisture from the wet wounded tissue, the bioadhesive rapidly and robustly adheres to the underlying tissue (fig. S1) and transits into a soft hydrogel in the rubbery state. Since the bioadhesive in the rubber state loses its ability to constrain the elastic recovery of the patch, the programmed strain in the patch is released to provide precisely-controlled mechanical contraction on the adhered wounded tissue (Fig. 1A). We perform systematic characterizations for the mechanical properties and adhesion performance of the strainprogrammable patch and develop theoretical and numerical models to establish design principles for the strain-programming and mechanical modulation of wounds. The biocompatibility of the strain-programmable patch is investigated based on in vitro and in vivo rat models. We further validate the efficacy of the proposed patch for programmed contraction of wounded tissues and 
accelerated healing of diabetic wounds based on in vivo diabetic mice and ex vivo human skin culture models.

\section{RESULTS}

\section{Design and mechanisms of the strain-programmable patch}

The strain-programmable patch consists of two layers: (i) a non-adhesive elastomer backing based on a hydrophilic thermoplastic polyurethane and (ii) a bioadhesive layer based on crosslinked networks of poly(acrylic acid) grafted with $N$-hydroxysuccinimide ester (PAA-NHS ester) and chitosan (Fig. 1A). The hydration-based shape-memory mechanism of the strain-programmable patch relies on a drastic change in the mechanical properties of the bioadhesive layer based on its hydration states (24-26) (see Supplementary Text for details on the hydration-based shape-memory mechanism). The hydrated bioadhesive in the rubbery state is soft (i.e., Young's modulus $\sim 40$ $\mathrm{kPa}$ ) and stretchable (i.e., over 4 times of the original length), whereas the dry bioadhesive becomes a glassy polymer with over 5 orders of magnitudes increase in stiffness (i.e., Young's modulus $\sim 5 \mathrm{GPa}$ ) (fig. S2). To program the strain in the patch, an assembly of the hydrated bioadhesive layer bonded with the elastomer backing is pre-stretched along with two in-plane directions (i.e., directions 1 and 2 in Fig. 1A) by ratios of $\lambda_{\text {patch }}^{\text {pre } 1}$ and $\lambda_{\text {patch }}^{\text {pre } 2}$, respectively. The prestretches on the assembly are maintained until the bioadhesive layer is dried to the glassy state. The glassy bioadhesive layer "freezes" the applied pre-stretches in itself (27-29) and constrains the elastomer backing from releasing the pre-stretches, due to the much higher rigidity of the glassy bioadhesive than the backing (Fig 1A and figs. S3 to S5; see Supplementary Text for details on the fabrication and strain-programming process).

Upon application of the strain-programmable patch on wet wounded tissues, the bioadhesive layer in the patch provides rapid robust adhesion to the wet wounded tissue surface within $5 \mathrm{~s}$ by absorbing the interfacial water between the patch and the tissue and forming crosslinks via the dry-crosslinking mechanism (23, 26) (fig. S1). Meanwhile, as the bioadhesive layer becomes hydrated, it quickly returns to the soft rubbery state within $30 \mathrm{~s}$, during which the strain-programmable patch shrinks along with the two in-plane directions by ratios of $\lambda_{\text {patch }}^{\text {shrink } 1}=$ $1 / \lambda_{\text {patch }}^{\text {pre1 }}$ and $\lambda_{\text {patch }}^{\text {shrink }}=1 / \lambda_{\text {patch }}^{\text {pre2 }}$, respectively (Fig. $1, \mathrm{~B}$ and D). This synergistic combination of the dry-crosslinking mechanism for rapid robust wet adhesion and the hydration-based shapememory mechanism for strain-programming enables facile and highly effective mechanical modulation of wet wounded tissues by the strain-programmable patch (fig. S6). Notably, the biaxial programmability of the strain-programmable patch endows it with broad applicability to various types of wounds including isotropic open wounds (i.e., $\lambda_{\text {patch }}^{\text {pre } 1}=\lambda_{\text {patch }}^{\text {pre }}=\lambda_{\text {patch }}^{\text {pre }}$; Fig. $1 \mathrm{C}$ and movie S1) as well as anisotropic incisional wounds (i.e., $\lambda_{\text {patch }}^{\text {pre1 }}=1$; Fig. $1 \mathrm{E}$ and movie S2).

\section{Mechanical properties}

The dry strain-programmable patch takes the form of a flexible thin dressing that can be applied to wet wounded tissues without any preparation process (Fig. 2, A and C). After adhering on tissues and releasing programmed strains, the swollen strain-programmable patch becomes a thin hydrogel layer with the tissue-like softness (Young's modulus $\sim 50 \mathrm{kPa}$ ), stretchability (over 3.5 
times of the original length) (Fig. 2, B and D), and high fracture toughness (over $400 \mathrm{~J} \mathrm{~m}^{-2}$ ) (fig. S7). Owing to the high programmability of the hydration-based shape-memory process, the contractile mechanical stresses generated by the strain-programmable patch can be precisely controlled based on the applied pre-stretches and the mechanical properties of the patch (Fig. 2, E and F; see Supplementary Text for details on the theoretical analysis). In particular, the mechanical properties and generated contractile stresses of the strain-programmable patch can be tuned by choosing various non-adhesive elastomer backing materials with different mechanical properties (fig. S8).

Furthermore, the robust interfacial integration between the non-adhesive elastomer backing and the bioadhesive layer in the swollen strain-programmable patch (interfacial toughness over $650 \mathrm{~J} \mathrm{~m}^{-2}$ ) provides mechanical stability and integrity in wet physiological environments $(30,31)$ (fig. S9). Notably, based on the multi-step pre-stretching fabrication process, the swelling ratio mismatch between the elastomer backing and the bioadhesive layer along the in-plane directions is canceled to minimize the geometric change and the interfacial delamination (figs. S3 and S4; see Supplementary Text for details on the fabrication and strain-programming process).

\section{Rapid, robust, and on-demand detachable adhesion}

To evaluate the adhesion performance of the strain-programmable patch on wet wounded tissues, we conduct four different types of mechanical tests following the testing standards for tissue adhesives to measure the interfacial toughness (by 180-degree peel test, ASTM F2256), shear strength (by lap-shear test, ASTM F2255), wound closure strength (by ASTM F2392-04), and burst strength (by ASTM F2392-04) based on wet porcine skin as the model tissue $(21,23)$ (fig. S10). The strain-programmable patch (with $\lambda_{\text {patch }}^{\text {pre }}=1.3$ ) can establish robust adhesion rapidly upon contact and gentle pressure $(1 \mathrm{kPa})$ application for less than $5 \mathrm{~s}$ with high interfacial toughness over $350 \mathrm{~J} \mathrm{~m}^{-2}$ (Fig. 2H), shear strength over $115 \mathrm{kPa}$ (Fig. 2I), wound closure strength over $7 \mathrm{~N}$ (Fig. 2J), and burst strength over $310 \mathrm{mmHg}$ (Fig. 2K) on wet tissues.

The adhesion performance of the strain-programmable patch outperforms that of commercially-available tissue adhesives and wound dressings including cyanoacrylate adhesives (e.g., Dermabond ${ }^{\circledR}$ ), polyethylene glycol-based adhesives (e.g., Coseal), fibrin-based adhesives (e.g., TachoSil ${ }^{\circledR}$ ), elastic wound dressings (e.g., Tegaderm ${ }^{\mathrm{TM}}$ ), and contractile wound dressings (e.g., Embrace ${ }^{\circledR}$ ) (Fig. 2, H to K). Furthermore, taking advantage of the triggerable detachment of the bioadhesive layer (32), the adhered strain-programmable patch can be atraumatically detached from the tissue on-demand by applying a biocompatible triggering solution (Fig. 2G, fig. S11, and movie S3). Such benign on-demand removal of the strain-programmable patch can potentially beneficial for the care of chronic diabetic wounds in clinical settings where frequent changes of wound dressings are often required $(33,34)$.

\section{In vitro and in vivo biocompatibility}

To determine the biocompatibility of the strain-programmable patch and its on-demand detachment process, we perform an in vitro cell viability assay based on mouse embryonic fibroblasts (mEFs) and an in vivo dorsal subcutaneous implantation based on a rat model (Fig. 3). The in vitro biocompatibility of the strain-programmable patch is comparable to that of the control media (DMEM), showing no statistically significant difference in in vitro cell viability for mEFs 
after 24-hour culture (Fig. 3A). The histological assessment made by a blinded pathologist indicates that the strain-programmable patch generates a mild to moderate inflammatory reaction, comparable to or lower than that generated by U.S. Food and Drug Administration (FDA)approved commercially-available tissue adhesives Coseal and Dermabond ${ }^{\circledR}(\mathrm{CA})$, respectively at 2 weeks after the implantation (Fig. 3, B to D and G). Furthermore, the triggerable detachment process of the strain-programmable patch generates a mild inflammatory reaction comparable to that generated by the sham control group (surgery without implantation) at 2 weeks post-surgery (Fig. 4, E to G).

\section{Programmable mechanical modulation of wound}

To provide quantitative guidelines for mechanical modulation of wounds by the strainprogrammable patch, we utilize both analytical solutions (figs. S12 and S13) and finite-element method (Fig. 4, A and B, and fig. S14) to model the wound contraction and the remodeling of stresses in the skin around the wound by the strain-programmable patch (see Supplementary Text for details on the analytical and finite-element modeling). Without loss of generality, we study a circular wound in the skin, which is a common form of diabetic wounds. In our models, we take into account a natively existing pre-strain and tension in the skin to better elucidate the mechanical modulation of wounds by the strain-programmable patch (35-37). Due to the native pre-strain and tension in the skin, the circular wound undergoes an initial enlargement in the diameter and a substantial increase in hoop stress (over 2 times of the native state) (Fig. 4D and fig. S13, B and D), yielding a stress concentration around the wound edge which can impair wound closure and healing especially in diabetic wounds (15-17) (Fig. 4E and fig. S13, C and E). We further validate the model with experiments on ex vivo diabetic mouse skin (Fig. 4, A to D) and ex vivo human skin (fig. S15, A to D) where the wound closure ratio predicted by the finite-element model shows close agreement with the experimental measurements.

The strain-programmable patch mechanically modulates the wound in diabetic mouse and human skin by (i) reducing the wound diameter (Fig. 4, B to D, fig. S15, B to D, and movie S4) and (ii) reducing the hoop stress around the wound edge (Fig. 4, B and E, fig. S15, C and E) to various degrees based on the relative size of the patch to the wound (Fig. 4D) and the amount of

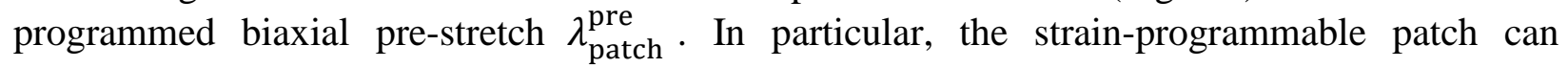
effectively reduce the hoop stress concentration around the wound edge at the lower pre-stretch amounts $\left(\lambda_{\text {patch }}^{\text {pre }}=1.1\right.$ and 1.2), and turn the hoop stress into compressive at the higher pre-stretch amount $\left(\lambda_{\text {patch }}^{\text {pre }}=1.3\right)$ (Fig. $4 \mathrm{E}$ and fig. S15E).

\section{Application on a diabetic mouse model of impaired wound healing}

From the above results, we hypothesize that the strain-programmable patch can potentially promote wound healing in diabetic mice at different time frames. In acute or short-term period, the strain-programmable patch can promote diabetic wound healing by applying mechanical contraction and subsequently approximating the wound edges right after the application on wet wounded tissues (Fig. 4, C and D, and movie S4). In chronic or long-term periods, the strainprogrammable patch can promote diabetic wound healing by providing a favorable mechanical environment through the remodeling of the stress state around the wound including the reduction in the hoop stress concentration (Fig. 4, B, E, and F). 
To assess the efficiency of the patch in vivo, we employed an established model of impaired diabetic wound healing; the $\mathrm{db} / \mathrm{db}$ mouse $(38,39)$. Application of the strain-programmable patch onto $6 \mathrm{~mm}$ dorsal excisional wounds resulted in markedly improved wound closure at both 5 and 10 days post-injury (D5 and D10), as evaluated by the \% of open wound, degree of reepithelialization and area of the migrating hyperproliferative neo-epidermis (Fig. 5, A to J) compared to the no strain patch and TD conditions. The strain patch wounds on D10 also displayed well-formed granulation tissue with thick collagen bundles and increased cellularity, which are indicative of advanced healing (Fig. 5D). In addition, strain patch treated wounds exhibited enhanced vascularization as evidenced by the higher density of CD31+ vessels (Fig. 6, A to D). There were also fewer active Caspase-3+ apoptotic cells in the wound bed of strain patch treated mice on D5 (fig. S16C) and more proliferating Ki67+ epidermal cells on D10 wounds (fig. S16E). Consistent with augmented blood vessel formation, extracellular matrix (ECM) production and rapid keratinocyte migration, the gene expression levels of several key pro-angiogenic and prohealing growth factors were elevated in the strain patch wounds, especially on D5, including Col3al, Tgfbl, Vegfa, Fgf2 and $H g f$ (fig. S17A, B, D, and E). Db/db mice have a well-documented deficiency in wound contraction capability (40) with dysregulated fibroblast to myofibroblast conversion throughout the healing duration (41), so we examined $\alpha$ SMA expression to determine if modifying the wound stress levels via the patch application influences myofibroblast levels (42). We found that the strain-programmable patch modified the presence of $\alpha \mathrm{SMA}+$ cells in the wounds, with significantly reduced numbers on D5, but increased on D10 (Fig. 6, E to H). This was accompanied by diminished expression of Engrailed-1 (En1) (fig. S17C and F), a transcription factor recently shown to define an important in wound repair dermal fibroblast subpopulation responsible for fibrosis (43-45). We hypothesize that initial strain-programmable patch application stress-shields the tissue and leads to decreased myofibroblasts, but as healing rapidly progresses newly deposited granulation tissue alters the mechanical properties of the wound and activates more $\alpha \mathrm{SMA}+$ cells on D10. Furthermore, adipocyte to myofibroblast differentiation (46) or the reverse (47) could also be implicated in the higher D10 levels observed as previously reported.

We then characterized the wound inflammatory cell infiltrate with multicolor flow cytometry (48) to profile the major immune cell types affected by the patch application. Our gating strategy is illustrated on figs. S18 and S19. In contrast with the strain and TD groups, there were more total immune cells (CD45+) in no strain patch treated wounds on D5 (fig. S20 A), including more neutrophils (CD45+CD64-Ly6G+) (fig. S20B) and monocytes (CD45+CD11b+CD64/intLy6C+) (fig. S20C), but fewer macrophages (CD45+CD11b+CD64+F4/80+) (fig. S20D) and T-cells (CD45+CD3+) (fig. S20F). The strain patch also induced an amplified immune response but to a moderate extent compared with the no strain patch. This is to be expected, as any interaction of a biomaterial with the immune system triggers an immune response (49). We also analyzed the expression of established macrophage polarization markers and discovered that on D5 the patch treated groups displayed an M1-skewed phenotype, with increased \% of CD80 and CD86 M1 macrophages (fig. S20I and J) and reduced \% of CD163 and CD301b M2 macrophages (fig. S20K and M). Interestingly, they also had higher \% of typically M2 associated CD206 cells (fig. S20L). On D10, there were more immune cells and neutrophils in the strain and no strain wounds (fig. S21A and B) and more monocytes and fewer macrophages in the no strain wounds (fig. S21C and D). The sustained increased number of monocytes and reduced macrophages denotes insufficient monocyte to macrophage differentiation and a prolonged inflammatory state. In addition, macrophages in the strain patch-treated wounds had a more M2-like phenotype with 
decreasing \% of CD80 (fig. S21J) and increasing \% of CD163 and CD301b (fig. S21K and M) suggesting that the healing was actively progressing towards the proliferation phase $(50)$.

Next, to better understand the mechanisms of the observed wound healing acceleration, we performed bulk RNA-seq on D10 wound tissues. Principal component analysis (PCA) revealed separate clusters of the samples according to treatment, indicating distinct transcriptome profiles (Fig. 7A). Differential gene expression analysis with $\log$ (fold change) $<1$ or $>1$ and false discovery rate $(\mathrm{FDR})<0.05$ on strain-programmable patch treated wounds versus Tegaderm only identified 3,581 significantly modified genes (1681 upregulated) (Fig. 7B) and 62 genes (14 upregulated) in strain-programmable versus no strain patch (Fig. 7C). The volcano plots and heat maps (fig. S22) illustrate the most highly expressed features, while the complete lists are presented in Data file S1. Further, overrepresentation analysis of the top differentially expressed genes highlighted the enrichment of multiple processes linked to muscle contraction which is in agreement with our observation of more $\alpha \mathrm{SMA}+$ cells in the strain-programmable patch wounds by D10 (Fig. 7, D to G). A similar analysis for no strain versus TD yielded cytokine related pathways as most enriched (fig. S23). Collectively, these findings indicate that applying the patch on diabetic murine cutaneous wounds promotes healing by positively affecting multiple integral reparative processes, including keratinocyte migration, angiogenesis and proliferation. It also alleviates the tension of the tissue leading to an initial diminished and subsequently increased myofibroblast presence which also proves beneficial for wound closure.

\section{Application on a human ex vivo model of wound healing}

To examine potential effects of the patch on human wound healing, we inflicted $6 \mathrm{~mm}$ punch biopsy wounds on panniculectomy derived discarded skin kept in cell culture conditions $(51,52)$ and monitored healing over four days (Fig. 8A and B). We quantified the distance between the two edges of the migrating epidermis as a measure of wound healing and found the strain patch to promote faster re-epithelialization compared to the other two conditions (Fig. 8C and E). Masson's trichrome staining (MTS) for the assessment of collagen fibers and scoring by an experienced pathologist demonstrated elevated intensity in the strain patch-treated wounds suggesting that the strain-programmable patch also influences the dermal ECM (Fig. 8D and F). We observed no differences in the number of fibroblast-like cells or vessels counted from hematoxylin and eosin (H\&E) stained wound sections (Fig. 8G and H).

To further explore the efficiency of the patch clinically and taking into consideration that DFU can be irregularly shaped, we utilized two representative examples of images taken from patients at the Joslin-Beth Israel Deaconess Foot Center and recreated similar wounds on ex vivo porcine skin (fig. S25A and B). Digital photography and FE simulation following the strainprogrammable patch application revealed that initial wound area was decreased and tension relief was achieved (fig. S25 C to F), which are encouraging insights into its future use in the clinic for treating DFU.

\section{DISCUSSION}

Our results show that application of the strain-programmable patch on $\mathrm{db} / \mathrm{db}$ mouse wounds achieves a $50 \%$ wound closure by day 5 post-injury and a remarkable $75 \%$ on day 10 and thus outperforms the previously reported interventions in similar models (53-55), including PDGF 
treatment (56) which is the only recombinant growth factor FDA-approved DFU therapy (57). Our findings that modulation of wound tension ameliorates healing complement and expand on the recent work by Mascharak et al. (44). They used both a device to control wound mechanical forces and a pharmacological inhibition strategy to demonstrate that Engrailed-1 expressing fibroblasts activated via mechanotransduction signaling are responsible for fibrosis in the wound and blocking them results in regeneration. Here, we show that a similar approach is also highly effective in impaired diabetic wound healing.

Profiling the major immune cell types involved in wound healing we discover that the strain-programmable patch elicits an inflammatory response compared to the Tegaderm control, which however does not prove detrimental to wound closure. Chronic diabetic wounds, like the $\mathrm{DFU}$, are mainly characterized by the persistence of low-grade inflammation and inability to progress to the next phase of wound healing $(18,58-60)$. Previous studies in our unit have shown that approaches that improve wound healing in diabetic murine models exert their beneficial effects by converting the low-grade chronic inflammation to an acute one and that this conversion is adequate to promote linear progression to the next phases and accelerate wound healing (61, 62). Similar findings have also been reported by us and others in human studies $(63,64)$.

FDA has approved four products for DFU treatment and all of them were developed in the 1990s: becaplermin (rhPDGF-BB), a recombinant growth factor (6); two bioengineered skin substitutes (Apligraf (4) and Dermagraft (5)); and Omnigraft that is based on Integra Dermal Regeneration Matrix (65). However, the efficacy of these products is rather limited, as in the pivotal trials, half or more of the participants failed to heal their wounds $(66,67)$ while their cost is considerable. Numerous other clinical trials with additional growth factors, including bFGF, EGF, and VEGF, devices, and other techniques have all failed to show meaningful efficacy (68). Furthermore, although basic research studies have indicated that DPP4 inhibitors may promote wound healing and reduce fibrosis (43), there is no concrete clinical evidence that these inhibitors can have any effect of DFU healing. This underscores the significant need to develop new therapies that could be tested in future clinical trials.

It has been well-established that mechanical reinforcement or stimuli to alleviate the adverse stress concentration around the wound can facilitate wound healing of healthy skin both in animal models and human clinical trials $(10-12,17,60,69)$. However, any potential therapeutic effects of such mechanical stimuli are underexplored in chronic diabetic wounds such as DFU with impaired wound healing including a reduced degree in contractility of wound edges and subsequent closure of wounds $(18,58-60,66)$. We hypothesize that a tissue adhesive biomaterial capable of providing programmed mechanical contractions can facilitate the healing of diabetic wounds by addressing this mechanical imbalance. To implement this hypothesis into a viable therapeutic platform, we develop the strain-programmable patch that synergistically incorporates the hydration-based shape-memory mechanism and the dry-crosslinking mechanism to achieve rapid, robust, and fully programmable mechanical modulation of wet wounded tissue. We provide a quantitative design guideline for the predictable and rational design of the proposed strainprogrammable patch based on theoretical, numerical, and experimental analysis and modeling. We further validate biocompatibility and diabetic wound healing efficacy of the strain-programmable patch via in vitro and in vivo rodent models. Taking advantage of the quantitative design guideline and fabrication process for the strain-programmable patch, we also demonstrate that the strainprogrammable patch can be readily translated into human-scale diabetic wound healing applications based on an ex vivo human skin wound healing model. 
Overall, our in vivo rodent and ex vivo human diabetic wound healing data validate our hypothesis and suggest that the strain-programmable patch can offer a promising therapeutic solution for the treatment of chronic diabetic wounds. We envision that the strain-programmable patch has the potential for commercialization and eventual translation as a clinical treatment for chronic diabetic wounds. While the current work provides systematically investigated platform technology and proof-of-concept efficacy of the therapeutic potential, there are future steps to be taken for further investigation and clinical translation. Future investigations should focus on employing multi-omics methods to comprehensively map the different cell populations and signaling pathways influenced by the strain programmable-patch treatment $(70,71)$. In addition, further studies are required to define applicability, frequency of application and suitable strain levels, among others, before use in a clinical setting. Completion of this work has the potential to lead to phase I/II clinical trials.

\section{MATERIALS AND METHODS}

\section{Study design}

The aim of this study was to develop a strain-programmable bioadhesive patch to provide an effective, reliable, and precisely-controllable mechanical modulation of wet diabetic wounds for accelerated healing. It was hypothesized that a bioadhesive material capable of both rapid robust adhesion on wet wounded tissues and programmable contraction would facilitate the healing of diabetic wounds by addressing their inherent mechanical imbalance. Mechanical characterizations were performed based on ex vivo porcine skin to optimize and evaluate the adhesion performance and strain-programmability of the proposed platform. In vitro tests were performed to evaluate the cytotoxicity of the strain-programmable patch. In vivo biocompatibility was evaluated by a blinded pathologist based on a rat subcutaneous implantation model. Diabetic wound healing efficacy of the strain-programmable patch was evaluated in vivo based on the $\mathrm{db} / \mathrm{db}$ mice wound healing model. Clinical translational potential was assessed with an ex vivo human skin culture model. The efficacy of diabetic wound healing in comparison with a standard care control group (Tegaderm ${ }^{\mathrm{TM}}$, 3M) was evaluated based on histology, immunohistochemistry, flow cytometry, RNA-seq and gene expression analyses.

\section{Preparation of the strain-programmable patch}

To prepare the bioadhesive layer, acrylic acid (30 w/w \%), chitosan (HMC+ Chitoscience Chitosan 95/500, $95 \%$ deacetylation, $2 \mathrm{w} / \mathrm{w} \%), \alpha$-ketoglutaric acid $(0.2 \mathrm{w} / \mathrm{w} \%)$, and poly(ethylene glycol methacrylate) (PEGDMA; $\mathrm{Mn}=550,0.03 \mathrm{w} / \mathrm{w} \%$ ) were dissolved in deionized water. Then, we dissolved $100 \mathrm{mg}$ functional monomer (NHS ester functionalized monomer with disulfide bond) synthesized following the previous report (32) in $1 \mathrm{ml}$ acetone and added to $10 \mathrm{ml}$ of the above stock solution to get a precursor solution. The precursor solution was then poured on a glass mold with spacers (the thickness is $210 \mu \mathrm{m}$ unless otherwise mentioned) and cured in ultraviolet light chamber (365 nm, $10 \mathrm{~W}$ power) for $30 \mathrm{~min}$. As a non-adhesive elastomer backing resin, $10 \mathrm{w} / \mathrm{w}$ $\%$ hydrophilic polyurethane (AdvanSource Biomaterials) in ethanol/water mixture (95:5 v/v) was spin-coated on the as-prepared bioadhesive at various speed (fig. S25, $100 \mathrm{rpm}$ was used otherwise mentioned). 
The as-prepared bioadhesive layer coated with the non-adhesive elastomer backing resin underwent multi-step processes to fabricate the strain-programmable patch (fig. S3). Detailed fabrication steps were described in the Supplementary Information. The prepared dry strainprogrammable patch was sealed in a plastic bag with desiccants (silica gel packets with active charcoal, McMaster Carr) and stored at $-20{ }^{\circ} \mathrm{C}$ before use. To prepare the triggering solution, 0.5 $\mathrm{M}$ sodium bicarbonate (SBC) and L-glutathione reduced (GSH) were dissolved in phosphate buffered saline (PBS). The triggering solution was filtered by using a $0.2-\mu \mathrm{m}$ sterile syringe filter before use.

\section{Mechanical characterization}

Unless otherwise indicated, the strain-programmable patch (with $\lambda_{\text {patch }}^{\text {pre }}=1.3$ ) was applied after washout of the tissue surfaces with PBS followed by $5 \mathrm{~s}$ pressing (with $1 \mathrm{kPa}$ pressure applied by either mechanical testing machine or equivalent weight). Unless otherwise indicated, all mechanical tests on adhesion samples were performed $12 \mathrm{~h}$ after initial pressing to ensure equilibrium swelling of the adhered strain-programmable patch in wet environments. The application of commercially-available tissue adhesives and wound dressings followed the provided manual for each product.

To measure interfacial toughness, adhered samples with widths of $2.5 \mathrm{~cm}$ were prepared and tested by the standard 180-degree peel test (ASTM F2256) using a mechanical testing machine (2.5-kN load-cell, Zwick/Roell Z2.5). All tests were conducted with a constant peeling speed of $50 \mathrm{~mm} \mathrm{~min}^{-1}$. The measured force reached a plateau as the peeling process entered the steady-state. Interfacial toughness was determined by dividing two times the plateau force by the width of the tissue sample (fig. S10A). Hydrophilic nylon filters (1 $\mu \mathrm{m}$ pore size, TISCH Scientific) were applied as a stiff backing for the strain-programmable patch. Poly(methyl methacrylate) (PMMA) films (with a thickness of $50 \mu \mathrm{m}$; Goodfellow) were applied using cyanoacrylate glue (Krazy Glue) as a stiff backing for the tissues. For on-demand detachment of the strain-programmable patch, the interfacial toughness was measured 5 min after applying the triggering solution.

To measure shear strength, the adhered samples with an adhesion area of $2.5 \mathrm{~cm}$ in width and $1 \mathrm{~cm}$ in length were prepared and tested by the standard lap-shear test (ASTM F2255) with a mechanical testing machine (2.5-kN load-cell, Zwick/Roell Z2.5) (fig. S10B). All tests were conducted with a constant tensile speed of $50 \mathrm{~mm} \mathrm{~min}^{-1}$. Shear strength was determined by dividing the maximum force by the adhesion area. Hydrophilic nylon filters were applied as a stiff backing for the strain-programmable patch. PMMA films were applied using cyanoacrylate glue (Krazy Glue) as a stiff backing for the tissues.

To measure wound closure strength, the adhered samples with $2.5 \mathrm{~cm}$ in width and $1 \mathrm{~cm}$ in overlap length (between adhesive and tissue) were prepared and tested by the standard wound closure strength test (ASTM F2458-05) with a mechanical testing machine (2.5-kN load-cell, Zwick/Roell Z2.5) (fig. S10C). All tests were conducted with a constant tensile speed of $50 \mathrm{~mm}$ $\min ^{-1}$. Wound closure strength was determined by measuring the maximum force.

To measure burst strength, the sealed samples with 3-mm hole were prepared and tested by the standard burst strength test (ASTM F2392-04) with a pressure gauge (Omega) (fig. S10D). All tests were conducted with a constant flow rate of PBS at $2 \mathrm{~mL} \mathrm{~min}^{-1}$. Burst strength was determined by measuring the maximum pressure. 
The tensile properties and fracture toughness of the strain-programmable patch were measured using pure-shear tensile tests of thin rectangular samples $(10 \mathrm{~mm}$ in length, $30 \mathrm{~mm}$ in width, and $0.5 \mathrm{~mm}$ in thickness) with a mechanical testing machine (20-N load-cell, Zwick/Roell Z2.5). All tests were conducted with a constant tensile speed of $50 \mathrm{~mm} \mathrm{~min}^{-1}$. The fracture toughness of the strain-programmable patch was calculated by following the previously reported method based on tensile tests of unnotched and notched samples (72) (fig. S7).

The tensile properties of ex vivo diabetic mouse skin and human skin were measured with a mechanical testing machine (2.5-kN load-cell, Zwick/Roell Z2.5). All tests were conducted with a constant tensile speed of $50 \mathrm{~mm} \mathrm{~min}^{-1}$. The nominal stress vs stretch curves of skin were fitted with the incompressible Ogden hyperelastic model as

$$
s=\frac{2 \mu}{\alpha \lambda}\left(\lambda^{\alpha}-\lambda^{-\frac{1}{2} \alpha}\right)
$$

where $s$ is nominal stress (i.e., the measured force divided by the cross-sectional area of an undeformed sample), $\mu$ is shear modulus, and $\alpha$ is Ogden coefficient. For diabetic mice skin, $\mu_{\text {mouse }}=87.5 \mathrm{kPa}$ and $\alpha_{\text {mouse }}=7$. For human skin, $\mu_{\text {human }}=40 \mathrm{kPa}$ and $\alpha_{\text {human }}=20$ (fig. S26).

\section{In vitro biocompatibility}

In vitro biocompatibility tests were conducted by using the strain-programmable patchconditioned media for cell culture (73). To prepare the strain-programmable patch-conditioned media for in vitro biocompatibility tests, $20 \mathrm{mg}$ of the strain-programmable patch was incubated in $1 \mathrm{~mL}$ Dulbecco's modified eagle medium (DMEM) at $37^{\circ} \mathrm{C}$ for $24 \mathrm{~h}$. The pristine DMEM was used as a control. Wild-type mouse embryonic fibroblasts (mEFs) were plated in 96-well plate $(N$ $=6$ per each case). The cells were then treated with the strain-programmable patch-conditioned media and incubated at $37{ }^{\circ} \mathrm{C}$ for $24 \mathrm{~h}$ in $5 \% \mathrm{CO}_{2}$. The cell viability was determined with a LIVE/DEAD viability/cytotoxicity kit for mammalian cells (Thermo Fisher Scientific) by adding $4 \mu \mathrm{M}$ calcein and ethidium homodimer-1 into the culture media. A confocal microscope (SP 8, Leica) was used to image live cells with excitation/emission at $495 \mathrm{~nm} / 515 \mathrm{~nm}$, and dead cells at $495 \mathrm{~nm} / 635 \mathrm{~nm}$, respectively. The cell viability was calculated by counting live (green fluorescence) and dead (red fluorescence) cells by using ImageJ.

\section{Ex vivo skin study}

All ex vivo experiments were reviewed and approved by the Committee on Animal Care at the Massachusetts Institute of Technology. For closure of isotropic porcine skin wounds, a hole was made on a porcine belly with an $8-\mathrm{mm}$ biopsy punch. For closure of anisotropic porcine skin wounds, a $3-\mathrm{cm}$ long laceration was made on a porcine belly with a scalpel. For closure of diabetic mouse skin wounds, a hole was made on a dorsal skin with a 6-mm biopsy punch. The strainprogrammable patch with $\lambda_{\text {patch }}^{\text {pre }}=1.3$ (for both biaxial and uniaxial pre-stretch) was used for $e x$ vivo study. 


\section{In vivo biocompatibility}

All animal surgeries were reviewed and approved by the Committee on Animal Care at the Massachusetts Institute of Technology. Female Sprague Dawley rats (225-250 g, Charles River Laboratories) were used for all in vivo studies. Before implantation, the strain-programmable patch was prepared using aseptic techniques and was further sterilized for $3 \mathrm{~h}$ under UV light. For implantation in the dorsal subcutaneous space, rats were anesthetized using isoflurane (1-2\% isoflurane in oxygen) in an anesthetizing chamber. Anesthesia was maintained using a nose cone. The back hair was removed and the animals were placed over a heating pad for the duration of the surgery. The subcutaneous space was accessed by a 1-2 cm skin incision per implant in the center of the animal's back. To create space for implant placement, blunt dissection was performed from the incision towards the animal shoulder blades. For the sham surgery group, no implant was placed in the subcutaneous pocket $(n=4)$. For the triggerable detachment group, the strainprogrammable patch $(10 \mathrm{~mm}$ in width and $20 \mathrm{~mm}$ in length) was placed in the subcutaneous pocket created above the incision and detached $5 \mathrm{~min}$ after applying $1 \mathrm{~mL}$ of the triggering solution $(n=$ 4). For the patch group, the strain-programmable patch (10 $\mathrm{mm}$ in width and $20 \mathrm{~mm}$ in length) was placed in the subcutaneous pocket created above the incision without detachment $(n=4)$. For commercially-available tissue adhesive groups, $0.5 \mathrm{~mL}$ of Coseal $(n=4)$ and Dermabond ${ }^{\circledR}$ cyanoacrylate adhesive $(n=4)$ were injected in the subcutaneous pocket created above the incision. The incision was closed using interrupted sutures (4-0 Vicryl, Ethicon) and 3-6 ml of saline were injected subcutaneously. Up to four implants were placed per animal ensuring no overlap between each subcutaneous pocket. After 2 weeks following the implantation, the animals were euthanized by $\mathrm{CO}_{2}$ inhalation. Subcutaneous regions of interest were excised and fixed in $10 \%$ formalin for $24 \mathrm{~h}$ for histological analyses.

Fixed tissue samples were placed into $70 \%$ ethanol and submitted for histological processing and hematoxylin and eosin (H\&E) staining at the Hope Babette Tang (1983) Histology Facility in the Koch Institute for Integrative Cancer Research at the Massachusetts Institute of Technology. Histological assessment was performed by a blinded pathologist on a scale of $0-4$ ( 0 , normal; 1 , mild; 2 , moderate; 3 , severe; 4 , very severe) to evaluate the degree of inflammation in the tissues surrounding the subcutaneous implants. The degree of acute inflammation was based on the number of neutrophils. The degree of chronic inflammation was based on the presence of lymphocytes, macrophages, and plasma cells. The degree of inflammation was evaluated based on the overall presence of indicators in each histological sample (normal, mild, moderate, severe, very severe). Representative images of each group were shown in the corresponding figures.

\section{In vivo diabetic mice wound healing study}

All procedures were approved by the BIDMC Institutional Animal Care and Use Committee. Male $\mathrm{db} / \mathrm{db}$ mice (stock \# 000642) were obtained from Jackson Laboratories and were acclimated to the animal facility for at least one week before surgery. They were routinely weighed and their blood glucose was assessed with a commercially available glucometer (Contour, Bayer) and confirmed to be $>250 \mathrm{mg} \mathrm{dL}^{-1}$. 12-week old mice were anesthetized using isoflurane and two circular biopsy punch 6-mm (Integra Miltex) full-thickness wounds were created on their depilated and disinfected dorsum. Sterile strain programmable patches, no strain patches or no patches were immediately placed on the wounds. The wounds were then covered with an occlusive dressing (Tegaderm, 3M) 
for protection. The mice were housed individually after surgery and observed every day until euthanized with excess $\mathrm{CO}_{2}$ on days 5 or 10 . The wounds were photographed on days 0,5 and 10 with a standard iphone 5 camera secured on a stand and measured with digital calipers (Thermo Fisher, 14-648-17). A ruler was placed beside the wounds as a scale bar for area calculation. Wound closure was quantified using both ImageJ and calipers measurements and expressed as percentage healed compared to day 0.

\section{Ex vivo human skin culture wound healing study}

Skin specimens were obtained from BioIVT (Westbury, NY) and derived from abdominoplasty procedures of three female patients aged 28 to 41 . De-identified samples were provided with removed subcutaneous adipose tissue in sterile PBS at $4^{\circ} \mathrm{C}$ on the day of surgery and were immediately processed. Prior to wounding, skin specimens were sterilized by sequential washes with iodine, $70 \%$ ethanol, and PBS and were then cut into evenly sized squares to fit into a $60 \mathrm{~mm}$ tissue culture dish (ThermoFisher). A $6 \mathrm{~mm}$ biopsy punch was used to gently punch the skin's epidermis partially penetrating into the dermis to create a wound. Strain-programmable patches, no strain patches or Tegaderm were applied onto the wounds. A sterile gauze was placed on top of the tissue culture dish and soaked with high glucose DMEM (ThermoFisher) supplemented with $10 \%$ heat inactivated FBS (Sigma-Aldrich) and 1\% Penicillin-Streptomycin (Gibco). Skin specimens were next placed dermis side down onto the culturing dishes and incubated at $37^{\circ} \mathrm{C}$, $5 \% \mathrm{CO}_{2}$. Media was changed every day.

\section{Histology immunohistochemistry and immunofluorescence}

Human or mouse wound tissues following completion of the study on days 4 or 5 and 10 respectively were bisected at the wound center. One half was snap-frozen and stored at $-20{ }^{\circ} \mathrm{C}$, while the other was fixed in $10 \%$ formalin and processed for paraffin embedding. $5 \mu \mathrm{m}$ thick sections were used. MTS and H\&E stains were performed at BIDMC Histology Core. Whole slide image acquisition was performed at the DF/HCC Research Pathology Cores with an Aperio CS2 scanner (Leica Biosystems). Re-epithelialization was quantified from MTS images by measuring the length of the migrating epithelial tongue covering the wound and dividing by the entire length of the wound. An experienced dermatopathologist (A.K.) scored the human wounds MTS slides according to intensity on a scale of 1 to 3 and counted the number of fibroblast-like cells and blood vessels. For immunohistochemistry of mouse wounds, tissue sections were deparaffinized, rehydrated and antigen retrieval was achieved with citrate buffer $\mathrm{pH} 6.0$ in a pressure cooker for $15 \mathrm{~min}$. They were then blocked in $1 \%$ BSA for $1.5 \mathrm{~h}$ at RT. The Vectastain Elite ABC Rabbit IgG Kit (Vector Laboratories) was used following the manufacturer's instructions. Sections were incubated with rabbit anti-active caspase-3 (1:40, BD, 559565) overnight at $4{ }^{\circ} \mathrm{C}$. Visualization of the secondary biotinylated antibody binding was performed using NovaRED substrate kit (Vector Laboratories). Images of sections were obtained at 10x magnification with Elipse E200 upright microscope (Nikon) using Motic Images Plus 3.0 software.

For immunofluorescence staining, deparaffinization and antigen retrieval of the paraffinembedded mice wound sections were performed as previously described. The primary antibodies used were: mouse monoclonal anti-Cytokeratin 14 (1:1000, Abcam ab7800), rat monoclonal antiKi67 (1:200, eBioscience, 14-5698-82), rabbit polyclonal anti-CD31 (1:50, Abcam ab28364) and 
goat polyclonal anti-alpha smooth muscle actin (1:100, Abcam ab21027). Sections were first blocked (5\% donkey serum, $1 \%$ BSA in $0.1 \%$ Triton-X PBS) for $1 \mathrm{hr}$ at RT and then incubated with primary antibodies overnight at $4{ }^{\circ} \mathrm{C}$ in a humidified chamber. The secondary antibodies used were all donkey at 1:500 dilution: anti-mouse IgG H\&L (Alexa Fluor® 488, ab150109); anti-rat IgG H\&L (Alexa Fluor® 647, ab150155), anti-rabbit IgG H\&L (Alexa Fluor® 594, ab150064); anti-goat IgG H\&L (Alexa Fluor® 488, ab150133); anti-rabbit IgG H\&L (Alexa Fluor® 594, ab150064); anti-foat IgG H\&L (Alexa Fluor® 647, ab150131). Nuclear counterstaining was performed with DAPI (Invitrogen). Sections were quenched for 5 minutes using the TrueView Autofluorescence Quenching kit to decrease background (Vector Laboratories) and covered with anti-fade mounting medium (Vector Laboratories). Images were obtained at 10x and 20x magnification with an Axio Imager A2 microscope using Zen Blue edition software (Zeiss). Quantification of the stainings was performed on Image J/FIJI by counting the positive cells/structures for a particular marker and dividing by the area of the tissue for normalization.

\section{Skin dissociation and flow cytometry}

Immediately following sacrifice, mouse skin comprising of wound and approximately $0.5 \mathrm{~mm}$ of peri-wound tissue was excised and kept on ice cold sterile PBS until processing within $2 \mathrm{hr}$. Four wounds from two mice were pooled as one sample to ensure enough single cells. The skin was finely minced with a scalpel and placed for $30 \mathrm{~min}$ at $37{ }^{\circ} \mathrm{C}$ on a shaker in a digesting enzyme cocktail of $2 \mathrm{mg} / \mathrm{ml}$ Collagenase P (Roche), $2 \mathrm{mg} / \mathrm{ml}$ Dispase (Gibco) and $1 \mathrm{mg} / \mathrm{ml}$ DNase I (Stemcell Technologies) in DMEM (Gibco) with 10\% FBS and 1\% P/S, using glass pipettes to break down the ECM every 10 min. Single cell suspensions were passed through a $40 \mu \mathrm{m}$ cell strainer, counted with a K2 cellometer (Nexcelom Bioscience) and cryopreserved in 90\% FBS $10 \%$ DMSO until processing.

Cells were quickly thawed and adjusted to a concentration of $10^{6}$ cells $/ \mathrm{mL}$. A LIVE/DEAD fixable dead cell stain kit was used to exclude dead cells from the analysis (ThermoFisher). AbC total antibody and amine reactive ArC compensation beads kits (ThermoFisher, A10497 and A10346) were included for single stain controls. After completing the viability stain per the manufacturer's instructions, cells were blocked (Biolegend FACS buffer with $0.05 \%$ anti-mouse CD16/32 Biolegend, 101320 and 0.05\% Tru-stain monocyte blocker Biolegend, 426103) for 10 min at RT. An antibody cocktail with details listed on Data file S2 was then added for 25 min on ice. Cells were washed $2 \mathrm{x}$, fixed with $0.4 \%$ PFA and stored at $4^{\circ} \mathrm{C}$ protected from light until analysis the next day. The samples were run on a CytoFLEX LX flow cytometer (Beckman Coulter) and data was processed and analyzed with CytExpert software (Beckman Coulter) at the BIDMC Flow Cytometry Core.

\section{RNA extraction, sequencing and analysis}

RNA extraction, library preparations, and sequencing reactions were conducted at GENEWIZ, LLC. (South Plainfield, NJ, USA). Total RNA was extracted using the Qiagen RNeasy Plus Universal mini kit following the manufacturer's instructions (Qiagen, Hilden, Germany). Extracted RNA samples were quantified using Qubit 2.0 Fluorometer (Life Technologies, Carlsbad, CA, USA) and RNA integrity was checked on Agilent TapeStation 4200 (Agilent Technologies, Palo Alto, CA, USA). RNA sequencing libraries were prepared using the NEBNext 
Ultra RNA Library Prep Kit for Illumina following manufacturer's instructions (NEB, Ipswich, MA, USA). Briefly, mRNAs were first enriched with Oligo(dT) beads. Enriched mRNAs were fragmented for $15 \mathrm{~min}$ at $94{ }^{\circ} \mathrm{C}$. First strand and second strand cDNAs were subsequently synthesized. cDNA fragments were end repaired and adenylated at 3 'ends, and universal adapters were ligated to cDNA fragments, followed by index addition and library enrichment by limitedcycle PCR. The sequencing libraries were validated on the Agilent TapeStation (Agilent Technologies, Palo Alto, CA, USA), and quantified by using Qubit 2.0 Fluorometer (Invitrogen, Carlsbad, CA) as well as by quantitative PCR (KAPA Biosystems, Wilmington, MA, USA). The sequencing libraries were clustered on 1 lane of a flow cell. After clustering, the flowcell was loaded on the Illumina HiSeq 4000 instrument and the samples were sequenced using a 2x150bp Paired End (PE) configuration. Image analysis and base calling were conducted by the HiSeq Control Software (HCS). Raw sequence data (.bcl files) generated from Illumina HiSeq were converted into fastq files and de-multiplexed using Illumina's bcl2fastq 2.17 software. One mismatch was allowed for index sequence identification.

Read quality was evaluated using FastQC (74) and data were pre-processed with Cutadapt (75) for adapter removal following best practices (76). Gene expression against the GRCm38 transcriptome (Ensembl 93 version) (77) was quantified with STAR (78) and featureCounts (79). Differential gene expression analysis was performed using DESeq2 (80), while ClusterProfiler (81) was utilized for functional enrichment investigations. Genes with log2 |Fold Change $\mid \geq 1$ and False Discovery Rate $(\mathrm{FDR}) \leq 0.05$ were considered statistically significant.

\section{Quantitative reverse transcription real-time PCR (qRT-PCR)}

The miScript II RT kit (Qiagen) was used according to manufacturer's protocol for cDNA synthesis from $1 \mu \mathrm{g}$ of RNA. $30 \mathrm{ng}$ of cDNA were used per PCR reaction. QuantiTect primers were all purchased from Qiagen: B2m (QT01149547); Collal (QT00162204); Col3al (QT01055516); Egf (QT00151018); En1 (QT00248248); Fgf2 (QT00128135); Fgf7 (QT00172004); Fnl (QT00135758); Hgf (QT00158046); Tgfbl (QT00145250); Vegfa (QT00160769). qRT-PCR was run with QuantiTect SYBR Green PCR kit (Qiagen) on a Stratagene Mx3005P with MxPro qPCR Software (Agilent Technologies). The cycling conditions used were according to the miScript SYBR Green PCR kit protocol (Qiagen). Quantification was performed using the $2-{ }^{\Delta \Delta \mathrm{Ct}}$ method. Gene expression was normalized against the housekeeping gene $B 2 m$.

\section{Statistical analysis}

MATLAB (version R2018b) and Graphpad Prism 7.04 were used to assess the statistical significance of all comparison studies in this work. Data distribution was assumed to be normal for all parametric tests, but not formally tested. In the statistical analysis for comparison between multiple samples, one-way ANOVA followed by Tukey's or Fisher's multiple comparison tests were conducted with the threshold of $* p \leq 0.05, * * p \leq 0.01$, and $* * * p \leq 0.001$. In the statistical analysis between two data groups, a two-sample Student's $t$-test was used, and the significance threshold was placed at $* p \leq 0.05, * * p \leq 0.01$, and $* * * p \leq 0.001$.

\section{SUPPLEMENTARY MATERIALS}




\section{Supplementary Texts}

Fig. S1. Rapid wet adhesion and triggerable detachment of a strain-programmable patch.

Fig. S2. Mechanical properties of elastomer backing and bioadhesive.

Fig. S3. Fabrication of the strain-programmable patch.

Fig. S4. Swelling of strain-programmable patch.

Fig. S5. Comparison between Flory-Rehner and neo-Hookean models.

Fig. S6. Isotropic and anisotropically strain-programming of the patch.

Fig. S7. Fracture toughness of strain-programmable patch.

Fig. S8. Various elastomer backings for the strain-programmable patch.

Fig. S9. Interfacial toughness between elastomer backing and bioadhesive in the strainprogrammable patch.

Fig. S10. Mechanical testing setups for evaluation of adhesion performance.

Fig. S11. Triggerable on-demand detachment of the strain-programmable patch.

Fig. S12. Schematic configuration for the analytical modeling.

Fig. S13. Comparison of analytical and finite-element analyses.

Fig. S14. Finite-element modeling for wound closure by the strain-programmable patch.

Fig. S15. Programmable closure and stress remodeling of ex vivo human skin wounds.

Fig. S16. Wound cells proliferation and apoptosis.

Fig. S17. RT-qPCR gene expression analysis.

Fig. S18. Gating strategy for the flow cytometry analysis of dissociated wound tissues.

Fig. S19. Additional gating strategy for T-cell subsets and polarized macrophages.

Fig. S20. Flow cytometry quantification of major immune cell populations and macrophage polarized states in D5 wounds.

Fig. S21. Flow cytometry quantification of major immune cell populations and macrophage polarized states in D10 wounds.

Fig. S22. Visualization of RNA sequencing results.

Fig. S23. No Strain vs TD differential expression and functional analyses.

Fig. S24. Applicability of the strain-programmable patch to irregularly-shaped wounds.

Fig. S25. Spin-coating of backing layer.

Fig. S26. Tensile properties of ex vivo human skin.

Data file S1. Complete lists of RNA-seq data.

Data file S2. Complete list of the antibody cocktail for flow cytometry.

Movie S1. Rapid adhesion and closure of an ex vivo porcine skin wound by an isotropically strainprogrammed patch. 
Movie S2. Rapid adhesion and closure of an ex vivo porcine skin wound by an anisotropically strain-programmed patch.

Movie S3. On-demand removal of an adhered patch on an ex vivo porcine skin wound by applying a triggering solution.

Movie S4. Rapid adhesion and closure of wound in an ex vivo diabetic mouse skin by a strainprogrammable patch.

\section{REFERENCES AND NOTES}

1. C. f. D. Control, Prevention, National diabetes statistics report, 2017. Atlanta, GA: Centers for Disease Control and Prevention, US Department of Health and Human Services 20, (2017).

2. L. S. Geiss et al., Resurgence of Diabetes-Related Nontraumatic Lower Extremity Amputation in the Young and Middle-Aged Adult U.S. Population. Diabetes Care, (2018).

3. A. J. Boulton, L. Vileikyte, G. Ragnarson-Tennvall, J. Apelqvist, The global burden of diabetic foot disease. Lancet 366, 1719-1724 (2005).

4. A. Veves, V. Falanga, D. G. Armstrong, M. L. Sabolinski, S. Apligraf Diabetic Foot Ulcer, Graftskin, a human skin equivalent, is effective in the management of noninfected neuropathic diabetic foot ulcers: a prospective randomized multicenter clinical trial. Diabetes Care 24, 290-295 (2001).

5. W. A. Marston, J. Hanft, P. Norwood, R. Pollak, The efficacy and safety of Dermagraft in improving the healing of chronic diabetic foot ulcers: results of a prospective randomized trial. Diabetes Care 26, 1701-1705 (2003).

6. T. J. Wieman, J. M. Smiell, Y. Su, Efficacy and safety of a topical gel formulation of recombinant human platelet-derived growth factor-BB (becaplermin) in patients with chronic neuropathic diabetic ulcers. A phase III randomized placebo-controlled doubleblind study. Diabetes Care 21, 822-827 (1998).

7. F. Tecilazich, T. Dinh, A. Veves, Treating diabetic ulcers. Expert opinion on pharmacotherapy 12, 593-606 (2011).

8. F. Tecilazich, T. L. Dinh, A. Veves, Emerging drugs for the treatment of diabetic ulcers. Expert opinion on emerging drugs 18, 207-217 (2013).

9. A. J. Singer, R. A. Clark, Cutaneous wound healing. The New England journal of medicine 341, 738-746 (1999).

10. I. George Broughton, J. E. Janis, C. E. Attinger, The basic science of wound healing. Plastic and Reconstructive Surgery 117, 12S-34S (2006).

11. I. George Broughton, J. E. Janis, C. E. Attinger, Wound healing: an overview. Plastic and Reconstructive Surgery 117, 1e-S-32e-S (2006).

12. G. C. Gurtner, S. Werner, Y. Barrandon, M. T. Longaker, Wound repair and regeneration. Nature 453, 314-321 (2008).

13. G. C. Gurtner et al., Improving cutaneous scar formation by controlling the mechanical environment: large animal and phase I studies. Annals of Surgery 254, 217-225 (2011).

14. V. W. Wong et al., A mechanomodulatory device to minimize incisional scar formation. Advances in Wound Care 2, 185-194 (2013).

15. S. A. Eming, P. Martin, M. Tomic-Canic, Wound repair and regeneration: mechanisms, signaling, and translation. Science translational medicine 6, 265sr266 (2014). 
16. L. A. Barnes et al., Mechanical forces in cutaneous wound healing: emerging therapies to minimize scar formation. Advances in Wound Care 7, 47-56 (2018).

17. H. I. C. Harn et al., The tension biology of wound healing. Experimental Dermatology 28, 464-471 (2019).

18. V. Falanga, Wound healing and its impairment in the diabetic foot. Lancet 366, 1736-1743 (2005).

19. M. A. Loots et al., Differences in cellular infiltrate and extracellular matrix of chronic diabetic and venous ulcers versus acute wounds. The Journal of investigative dermatology 111, 850-857 (1998).

20. H. Brem, M. Tomic-Canic, Cellular and molecular basis of wound healing in diabetes. The Journal of clinical investigation 117, 1219-1222 (2007).

21. J. Li et al., Tough adhesives for diverse wet surfaces. Science 357, 378-381 (2017).

22. S. Blacklow et al., Bioinspired mechanically active adhesive dressings to accelerate wound closure. Science Advances 5, eaaw3963 (2019).

23. H. Yuk et al., Dry double-sided tape for adhesion of wet tissues and devices. Nature 575, 169-174 (2019).

24. F. N. Kelley, F. Bueche, Viscosity and glass temperature relations for polymer-diluent systems. Journal of Polymer Science 50, 549-556 (1961).

25. H. Frisch, T. Wang, T. Kwei, Diffusion in glassy polymers. II. Journal of Polymer Science Part A-2: Polymer Physics 7, 879-887 (1969).

26. X. Mao, H. Yuk, X. Zhao, Hydration and swelling of dry polymers for wet adhesion. Journal of the Mechanics and Physics of Solids, 103863 (2020).

27. A. Lendlein, R. Langer, Biodegradable, elastic shape-memory polymers for potential biomedical applications. Science 296, 1673-1676 (2002).

28. P. T. Mather, X. Luo, I. A. Rousseau, Shape memory polymer research. Annual Review of Materials Research 39, 445-471 (2009).

29. H. Meng, G. Li, A review of stimuli-responsive shape memory polymer composites. Polymer 54, 2199-2221 (2013).

30. H. Yuk, T. Zhang, S. Lin, G. A. Parada, X. Zhao, Tough bonding of hydrogels to diverse non-porous surfaces. Nature Materials 15, 190-196 (2016).

31. H. Yuk, T. Zhang, G. A. Parada, X. Liu, X. Zhao, Skin-inspired hydrogel-elastomer hybrids with robust interfaces and functional microstructures. Nature communications 7 , 1-11 (2016).

32. X. Chen, H. Yuk, J. Wu, C. S. Nabzdyk, X. Zhao, Instant tough bioadhesive with triggerable benign detachment. Proceedings of the National Academy of Sciences, (2020).

33. D. Upton, K. Solowiej, C. Hender, K. Woo, Stress and pain associated with dressing change in patients with chronic wounds. Journal of Wound Care 21, 53-61 (2012).

34. G. Han, R. Ceilley, Chronic wound healing: a review of current management and treatments. Advances in Therapy 34, 599-610 (2017).

35. C. Flynn, A. Taberner, P. Nielsen, Mechanical characterisation of in vivo human skin using a 3D force-sensitive micro-robot and finite element analysis. Biomechanics and Modeling in Mechanobiology 10, 27-38 (2011).

36. A. B. Berezovsky et al., Primary contraction of skin grafts: a porcine preliminary study. Plast Aesthet Res Serial Online 25, 22-26 (2015). 
37. H. Joodaki, M. B. Panzer, Skin mechanical properties and modeling: a review. Proceedings of the Institution of Mechanical Engineers, Part H: Journal of Engineering in Medicine 232, 323-343 (2018).

38. A. Grada, J. Mervis, V. Falanga, Research Techniques Made Simple: Animal Models of Wound Healing. J Invest Dermatol 138, 2095-2105 e2091 (2018).

39. S. S. Scherer et al., Wound healing kinetics of the genetically diabetic mouse. Wounds 20, 18-28 (2008).

40. L. Chen, R. Mirza, Y. Kwon, L. A. DiPietro, T. J. Koh, The murine excisional wound model: Contraction revisited. Wound Repair Regen 23, 874-877 (2015).

41. X. T. Wang, C. C. McKeever, P. Vonu, C. Patterson, P. Y. Liu, Dynamic Histological Events and Molecular Changes in Excisional Wound Healing of Diabetic DB/DB Mice. $J$ Surg Res 238, 186-197 (2019).

42. B. Hinz, Formation and function of the myofibroblast during tissue repair. $J$ Invest Dermatol 127, 526-537 (2007).

43. Y. Rinkevich et al., Skin fibrosis. Identification and isolation of a dermal lineage with intrinsic fibrogenic potential. Science 348, aaa2151 (2015).

44. S. Mascharak et al., Preventing Engrailed-1 activation in fibroblasts yields wound regeneration without scarring. Science 372, (2021).

45. D. Jiang et al., Two succeeding fibroblastic lineages drive dermal development and the transition from regeneration to scarring. Nat Cell Biol 20, 422-431 (2018).

46. B. A. Shook et al., Dermal Adipocyte Lipolysis and Myofibroblast Conversion Are Required for Efficient Skin Repair. Cell Stem Cell 26, 880-895 e886 (2020).

47. M. V. Plikus et al., Regeneration of fat cells from myofibroblasts during wound healing. Science 355, 748-752 (2017).

48. N. Joshi et al., Comprehensive characterization of myeloid cells during wound healing in healthy and healing-impaired diabetic mice. Eur J Immunol 50, 1335-1349 (2020).

49. E. Mariani, G. Lisignoli, R. M. Borzi, L. Pulsatelli, Biomaterials: Foreign Bodies or Tuners for the Immune Response? Int J Mol Sci 20, (2019).

50. P. Krzyszczyk, R. Schloss, A. Palmer, F. Berthiaume, The Role of Macrophages in Acute and Chronic Wound Healing and Interventions to Promote Pro-wound Healing Phenotypes. Front Physiol 9, 419 (2018).

51. O. Stojadinovic, M. Tomic-Canic, Human ex vivo wound healing model. Methods Mol Biol 1037, 255-264 (2013).

52. J. Gherardini, M. van Lessen, I. Piccini, J. Edelkamp, M. Bertolini, Human Wound Healing Ex Vivo Model with Focus on Molecular Markers. Methods Mol Biol 2154, 249-254 (2020).

53. S. A. Castleberry et al., Self-Assembled Wound Dressings Silence MMP-9 and Improve Diabetic Wound Healing In Vivo. Adv Mater 28, 1809-1817 (2016).

54. S. Shibata et al., Adiponectin regulates cutaneous wound healing by promoting keratinocyte proliferation and migration via the ERK signaling pathway. J Immunol 189, 3231-3241 (2012).

55. M. Gao et al., Acceleration of diabetic wound healing using a novel protease-anti-protease combination therapy. Proc Natl Acad Sci U S A 112, 15226-15231 (2015).

56. R. L. Brown, M. P. Breeden, D. G. Greenhalgh, PDGF and TGF-alpha act synergistically to improve wound healing in the genetically diabetic mouse. J Surg Res 56, 562-570 (1994). 
57. J. M. Smiell et al., Efficacy and safety of becaplermin (recombinant human platelet-derived growth factor-BB) in patients with nonhealing, lower extremity diabetic ulcers: a combined analysis of four randomized studies. Wound Repair Regen 7, 335-346 (1999).

58. M. A. Loots et al., Differences in cellular infiltrate and extracellular matrix of chronic diabetic and venous ulcers versus acute wounds. The Journal of investigative dermatology 111, 850-857 (1998).

59. H. Brem, M. Tomic-Canic, Cellular and molecular basis of wound healing in diabetes. The Journal of clinical investigation 117, 1219-1222 (2007).

60. S. A. Eming, P. Martin, M. Tomic-Canic, Wound repair and regeneration: mechanisms, signaling, and translation. Science translational medicine 6, 265sr266 (2014).

61. E. C. Leal et al., Substance $\mathrm{P}$ promotes wound healing in diabetes by modulating inflammation and macrophage phenotype. Am J Pathol 185, 1638-1648 (2015).

62. A. Tellechea et al., Topical Application of a Mast Cell Stabilizer Improves Impaired Diabetic Wound Healing. J Invest Dermatol 140, 901-911 e911 (2020).

63. R. C. Stone et al., A bioengineered living cell construct activates an acute wound healing response in venous leg ulcers. Science translational medicine 9, (2017).

64. G. Theocharidis et al., Integrated Skin Transcriptomics and Serum Multiplex Assays Reveal Novel Mechanisms of Wound Healing in Diabetic Foot Ulcers. Diabetes 69, 21572169 (2020).

65. V. R. Driver et al., A clinical trial of Integra Template for diabetic foot ulcer treatment. Wound repair and regeneration : official publication of the Wound Healing Society [and] the European Tissue Repair Society 23, 891-900 (2015).

66. F. Tecilazich, T. Dinh, A. Veves, Treating diabetic ulcers. Expert opinion on pharmacotherapy 12, 593-606 (2011).

67. F. Tecilazich, T. L. Dinh, A. Veves, Emerging drugs for the treatment of diabetic ulcers. Expert opinion on emerging drugs 18, 207-217 (2013).

68. D. Baltzis, I. Eleftheriadou, A. Veves, Pathogenesis and treatment of impaired wound healing in diabetes mellitus: new insights. Adv Ther 31, 817-836 (2014).

69. A. J. Singer, R. A. Clark, Cutaneous wound healing. The New England journal of medicine 341, 738-746 (1999).

70. G. Theocharidis et al., Single Cell Transcriptomic Landscape of Diabetic Foot Ulcers. bioRxiv, 2021.2003.2011.434413 (2021).

71. M. Asp, J. Bergenstrahle, J. Lundeberg, Spatially Resolved Transcriptomes-Next Generation Tools for Tissue Exploration. Bioessays 42, e1900221 (2020).

72. J.-Y. Sun et al., Highly stretchable and tough hydrogels. Nature 489, 133-136 (2012).

73. M. C. Darnell et al., Performance and biocompatibility of extremely tough alginate/polyacrylamide hydrogels. Biomaterials 34, 8042-8048 (2013).

74. S. Andrews. (Babraham Bioinformatics, Babraham Institute, Cambridge, United Kingdom, 2010).

75. M. Martin, Cutadapt removes adapter sequences from high-throughput sequencing reads. EMBnet. Journal 17, 10-12 (2011).

76. A. Conesa et al., A survey of best practices for RNA-seq data analysis. Genome Biology 17, 13 (2016).

77. F. Cunningham et al., Ensembl 2019. Nucleic Acids Research 47, D745-D751 (2019).

78. A. Dobin et al., STAR: ultrafast universal RNA-seq aligner. Bioinformatics 29, 15-21 (2013). 
79. Y. Liao, G. K. Smyth, W. Shi, featureCounts: an efficient general purpose program for assigning sequence reads to genomic features. Bioinformatics 30, 923-930 (2014).

80. M. I. Love, W. Huber, S. Anders, Moderated estimation of fold change and dispersion for RNA-seq data with DESeq2. Genome Biology 15, 550 (2014).

81. G. Yu, L.-G. Wang, Y. Han, Q.-Y. He, clusterProfiler: an R package for comparing biological themes among gene clusters. Omics: A Journal of Integrative Biology 16, 284287 (2012).

82. C. Liu, H. Qin, P. Mather, Review of progress in shape-memory polymers. Journal of Materials Chemistry 17, 1543-1558 (2007).

83. P. J. Flory, J. Rehner Jr, Statistical mechanics of cross-linked polymer networks I. Rubberlike elasticity. The Journal of Chemical Physics 11, 512-520 (1943).

84. P. J. Flory, Principles of polymer chemistry. (Cornell University Press, 1953).

85. N. Bouklas, R. Huang, Swelling kinetics of polymer gels: comparison of linear and nonlinear theories. Soft Matter 8, 8194-8203 (2012).

86. S. Cai, Z. Suo, Equations of state for ideal elastomeric gels. Europhysics Letters 97, 34009 (2012).

87. O. H. Yeoh, Some forms of the strain energy function for rubber. Rubber Chemistry and Technology 66, 754-771 (1993).

88. X. Liang, F. Tao, S. Cai, Creasing of an everted elastomer tube. Soft Matter 12, 7726-7730 (2016).

Acknowledgments: The authors thank the Koch Institute Swanson Biotechnology Center for technical support, specifically K. Cormier and the Histology Core for the histological processing and analysis. Funding: This work is supported by Defense Advanced Research Projects Agency (DARPA) (5(GG0015670)). A.V. received funding from the National Rongxiang Xu Foundation. G.T. received a George and Marie Vergottis Foundation Postdoctoral Fellowship. H.Y. acknowledges the financial support from Samsung Scholarship. H.R. acknowledges the financial support from Kwanjeong Educational Foundation Scholarship. Author contributions: G.T., H.Y., A.V., and X.Z. designed the study. H.Y. conceived the idea for the strain-programmable patch. H.Y. and H.R. developed the materials and method for the strain-programmable patch. H.Y., H.R., C.S.N. designed the in vitro and ex vivo experiment. H.Y. and H.R. conducted the in vitro and ex vivo experiment and analysis. L.W. and C.F.G. designed and conducted the theoretical and numerical modeling and analysis. J.W. and H.Y. designed and conducted the in vivo biocompatibility experiment. G.T., L.C. and A.V. designed and conducted the in vivo diabetic wound healing experiment and analysis. G.T., I.M. and N.J. completed the flow cytometry, histology, immunofluorescence, gene expression and human skin ex vivo experiment analyses. A.K. performed histology assessment and scoring. X.L.K. and I.S.V. performed sequencing analysis. G.T., H.Y., X.Z., and A.V. wrote the manuscript with inputs from all authors. Competing interests: H.Y., H.R., X.Z., G.T., and A.V. are the inventors of a patent application (U.S. Application No.63/148,901) that covers the design and mechanism of the strainprogrammable patch for diabetic wound healing. Data and materials availability: All data is available in the main text or the Supplementary Information. The RNA-seq data generated in the present study were deposited in the Gene Expression Omnibus (GEO) with accession number 'GSE154132'. 


\section{FIGURES}

A
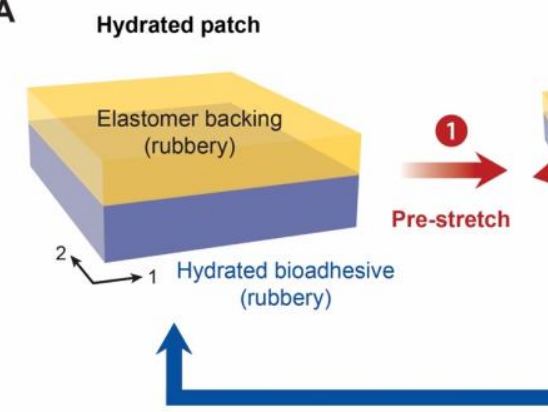

B

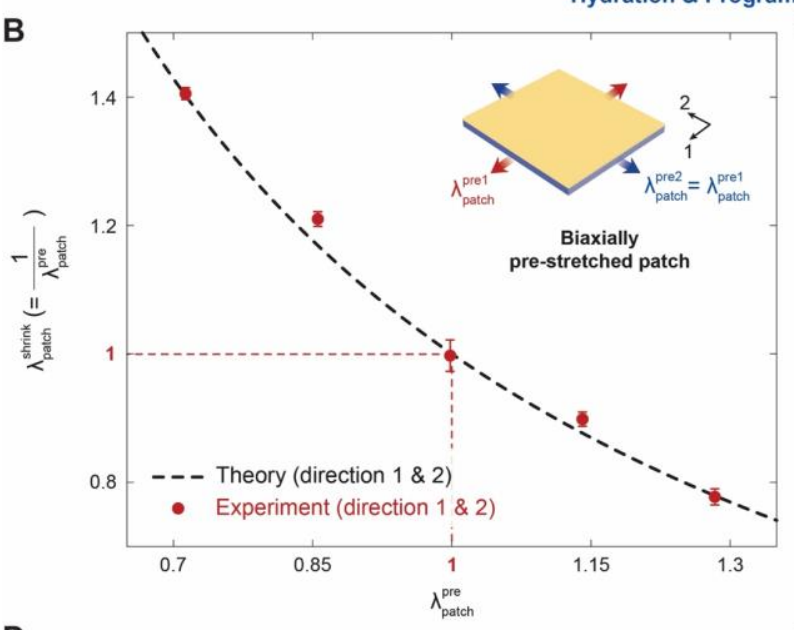

D

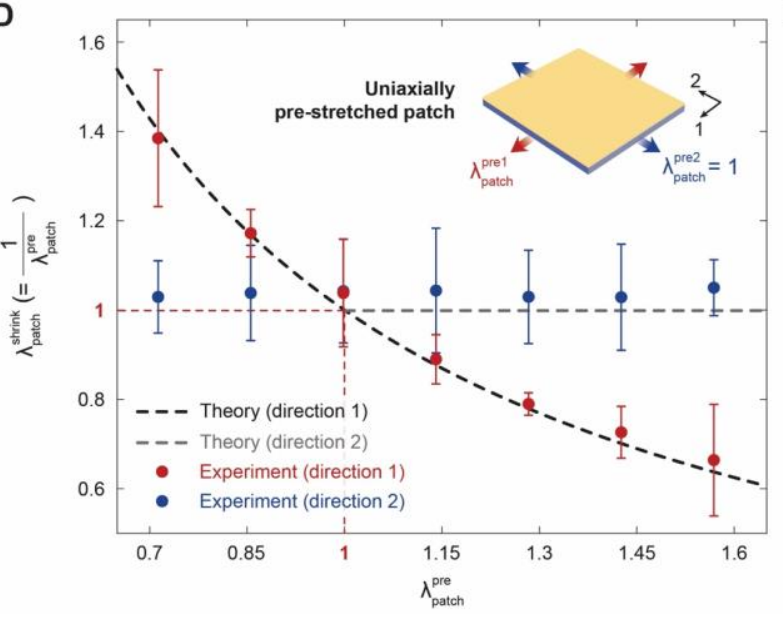

3
Pre-stretched patch

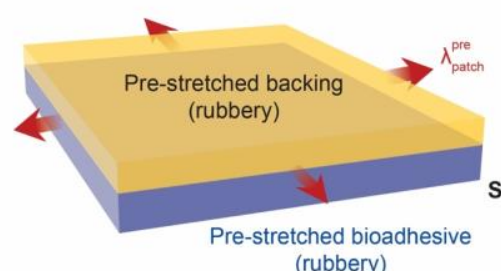

(rubbery)
Dry strain-programmable patch

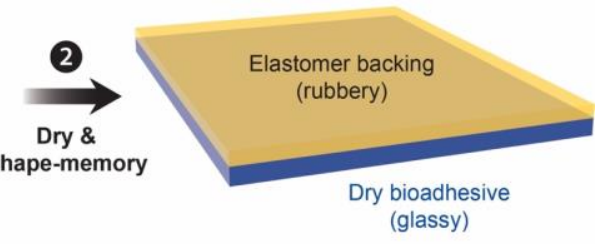

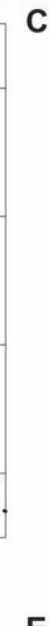
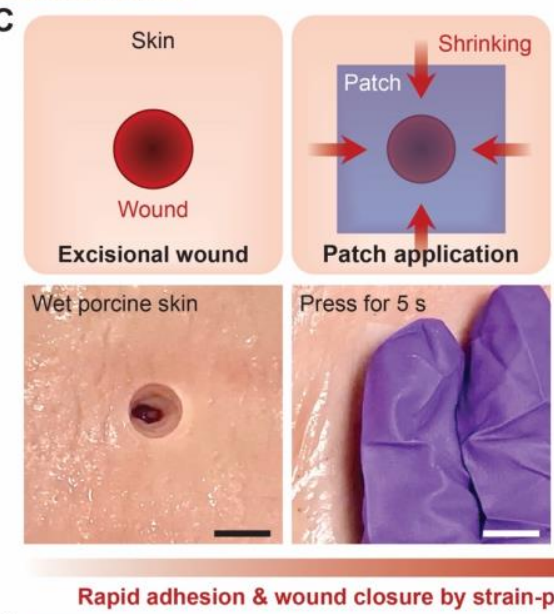

Adhered \& shrunken patch

Rapid adhesion \& wound closure by strain-programmable patch
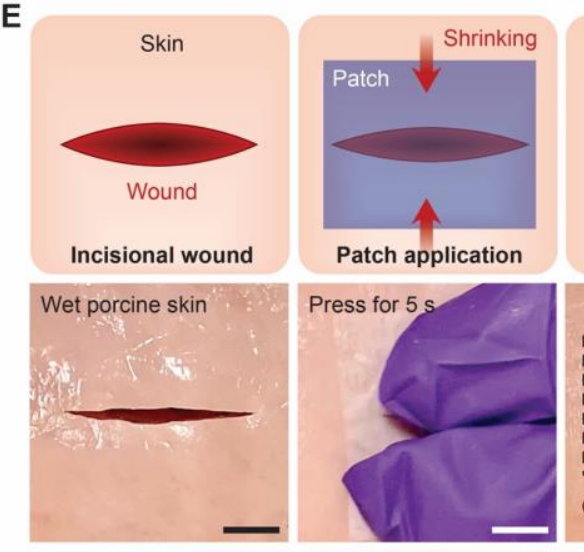

Adhered \& shrunken patch

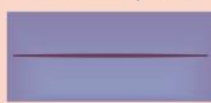

Wound closure \& Stress remodeling

Rapid adhesion \& wound closure by strain-programmable patch

Fig. 1. Design and mechanism of the strain-programmable patch. (A) Strain programming of the patch by a hydration-based shape-memory mechanism. (B) Theoretical and experimental values of $\lambda_{\text {patch }}^{\text {shrink }}$ vs. $\lambda_{\text {patch }}^{\text {pre }}$ for isotropically strain-programmed patches. (C) Closure of a circular wound in a porcine skin by an isotropically strain-programmed patch. (D) Theoretical and experimental values of $\lambda_{\text {patch }}^{\text {shrink }}$ vs. $\lambda_{\text {patch }}^{\text {pre }}$ for anisotropically strain-programmed patches. (E) Closure of an incisional wound in a porcine skin by an anisotropically strain-programmed patch. Values in B and D represent the means $\pm \mathrm{SD}(n=4)$. Scale bars, $10 \mathrm{~mm}(\mathrm{C}$ and $\mathrm{E})$. 

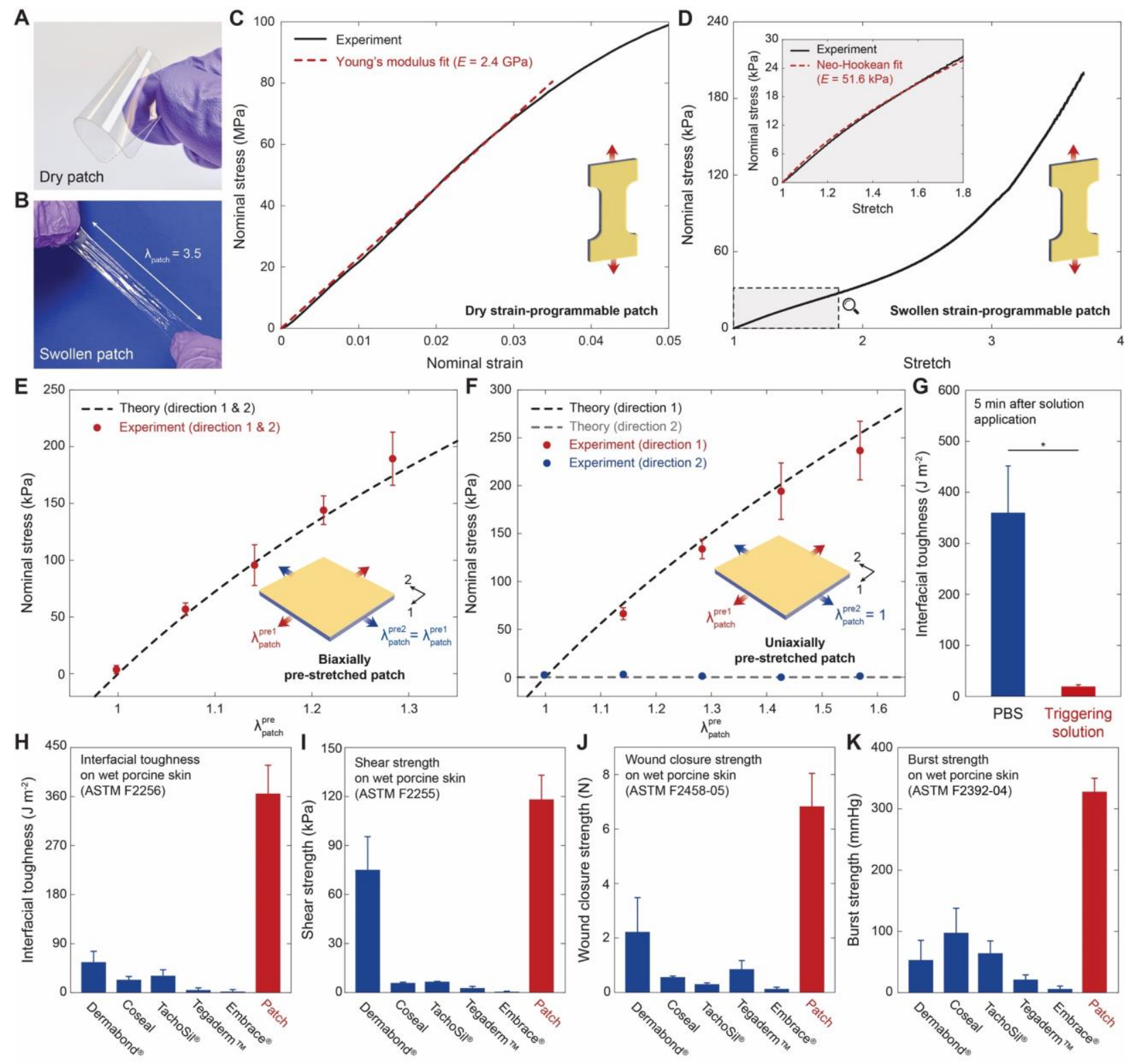

G 600

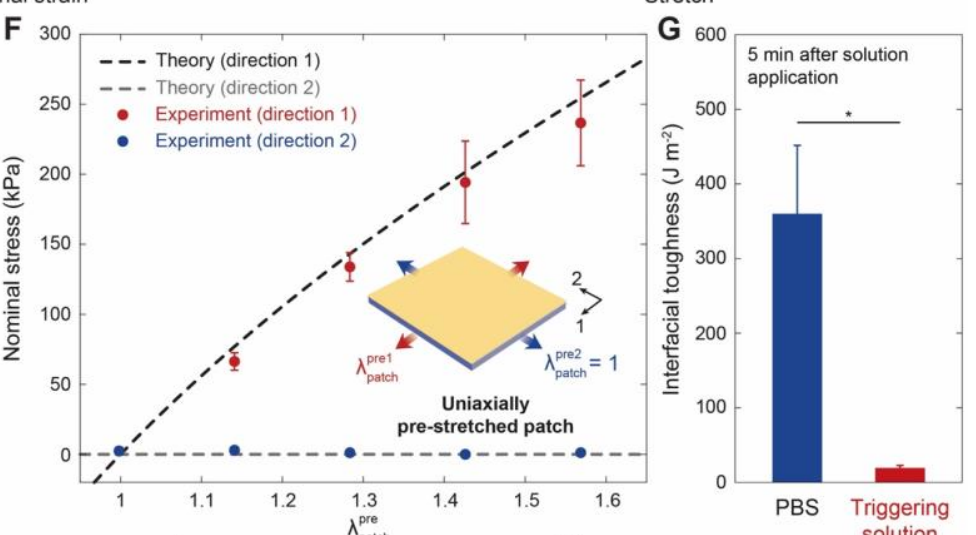

Fig. 2. Mechanical properties and adhesion performance. (A and B) Images of a dry (A) and a swollen (B) strain-programmable patch. (C and D) Nominal stress vs. stretch curves for a dry (C) and a swollen (D) strain-programmable patch. (E and $\mathbf{F}$ ) Experiment and theory values for the nominal contractile stress vs. $\lambda_{\text {patch }}^{\text {pre }}$ generated by the isotropically (E) and the anisotropically $(F)$ strain-programmed patches. (G) Interfacial toughness of the strain-programmable patch on wet porcine skin 5 min after applying PBS and the triggering solution. (H to $\mathbf{K}$ ) Interfacial toughness (measured by ASTM F2256) (H), shear strength (measured by ASTM F2255) (I), wound closure strength (measured by ASTM F2458-05) (J), and burst strength (measured by F2392-04) (K) of the strain-programmable patch on wet porcine skin. Values in $\mathrm{G}$ to K represent the means $\pm \mathrm{SD}(n$ $=4) . P$ values are determined by a Student's $t$ test; ns, not significant; $* p \leq 0.05$. 

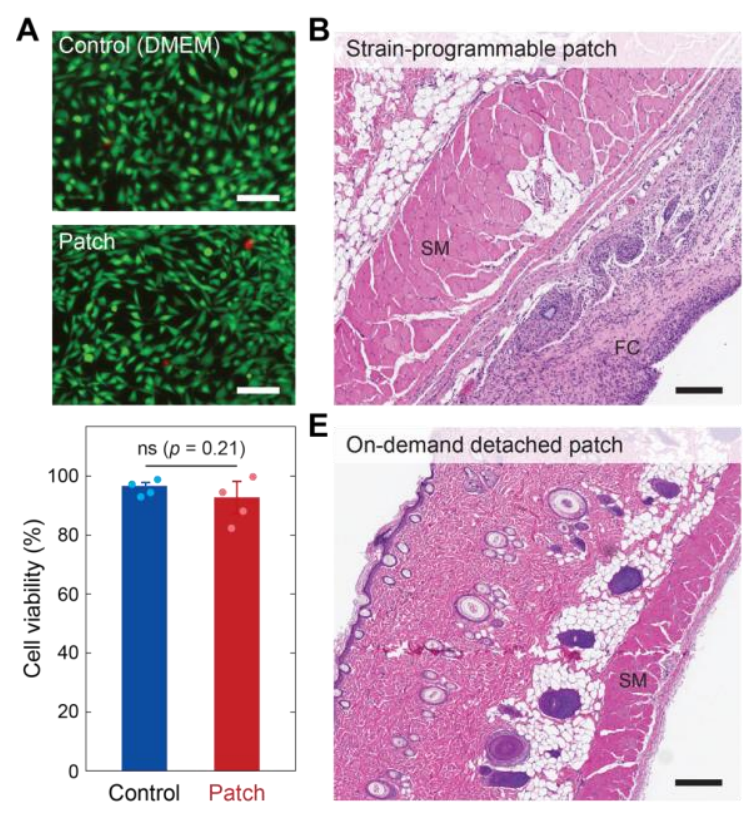

E

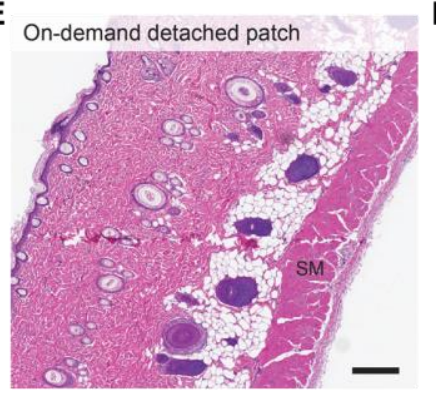

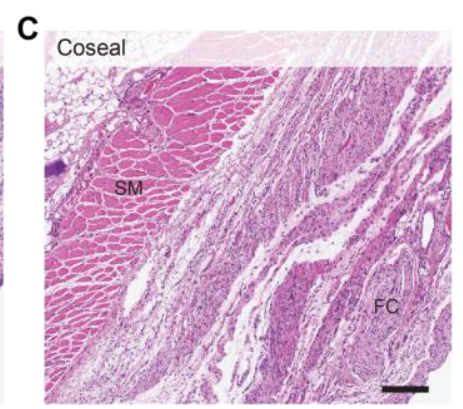

$\mathbf{F}$

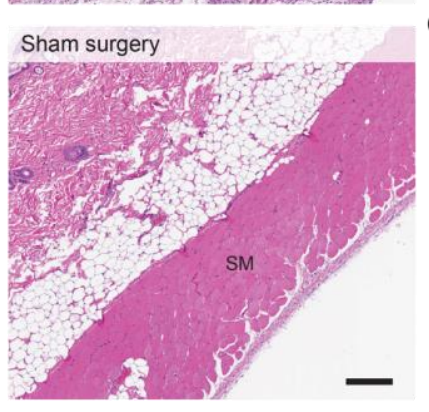

D
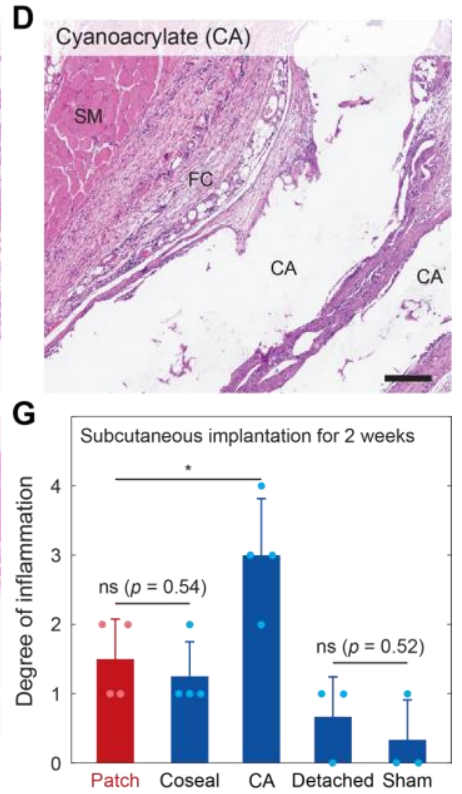

Fig. 3. In vitro and in vivo biocompatibility. (A) Representative LIVE/DEAD assay images and the cell viability of mouse embryonic fibroblasts (mEFs) for control (DMEM) and the strainprogrammable patch after 24-hour culture. (B to F) Representative histological images for the subcutaneously implanted strain-programmable patch (B), Coseal (C), Dermabond ${ }^{\circledR}$ cyanoacrylate (CA) adhesive (D), strain-programmable patch after on-demand detachment (E), and sham surgery (F) for 2 weeks stained with hematoxylin and eosin (H\&E). (G) Degree of inflammation of various groups evaluated by a blinded pathologist ( 0 , normal; 1 , mild; 2 , moderate; 3 , severe; 4 , very severe) after 2 weeks of subcutaneous implantation. SM and FC indicate skeletal muscle and fibrous capsule, respectively. All experiments are repeated four times with similar results. Values in A and G represent the means $\pm \mathrm{SD}(n=4)$. $P$ values are determined by Student's $t$ test; ns, not significant; $* p \leq 0.05$. Scale bars, $200 \mu \mathrm{m}$ (A to F). 
A

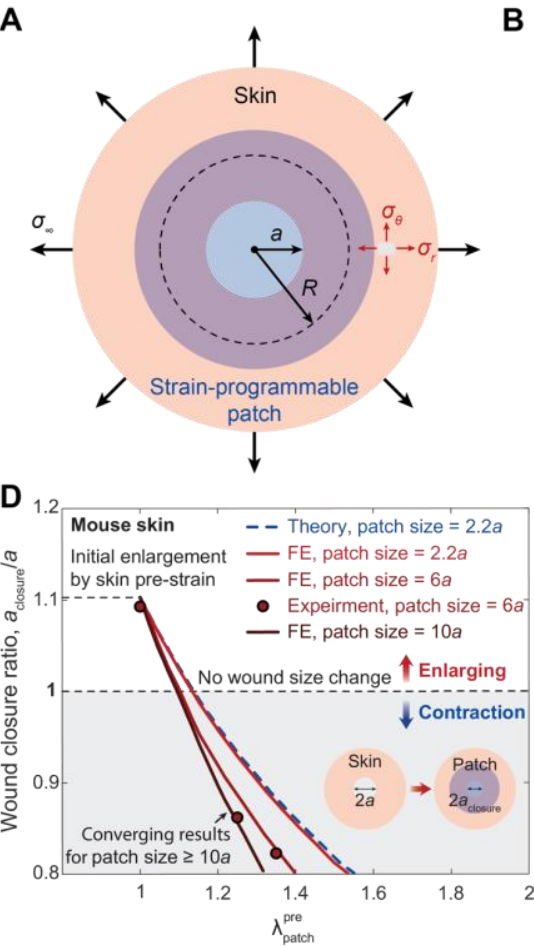

B

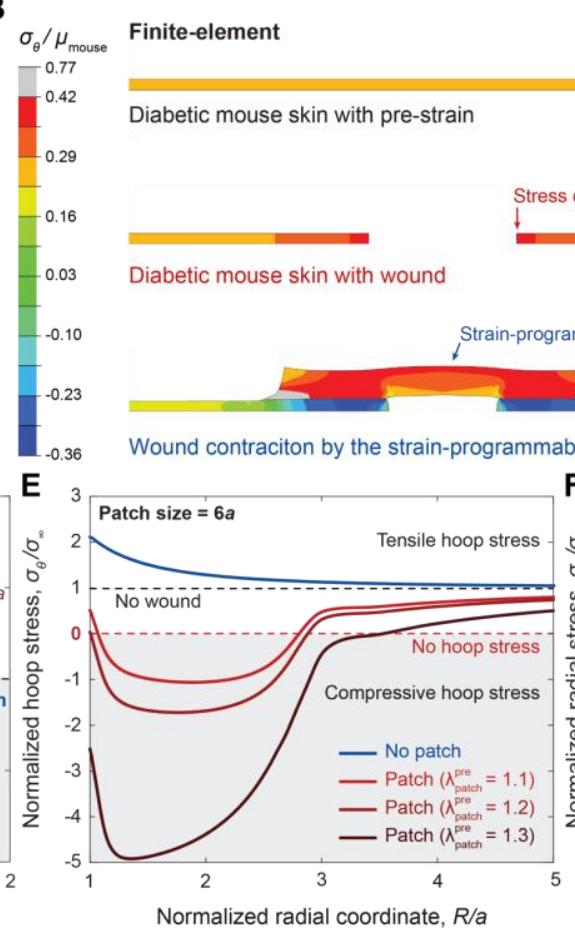

C
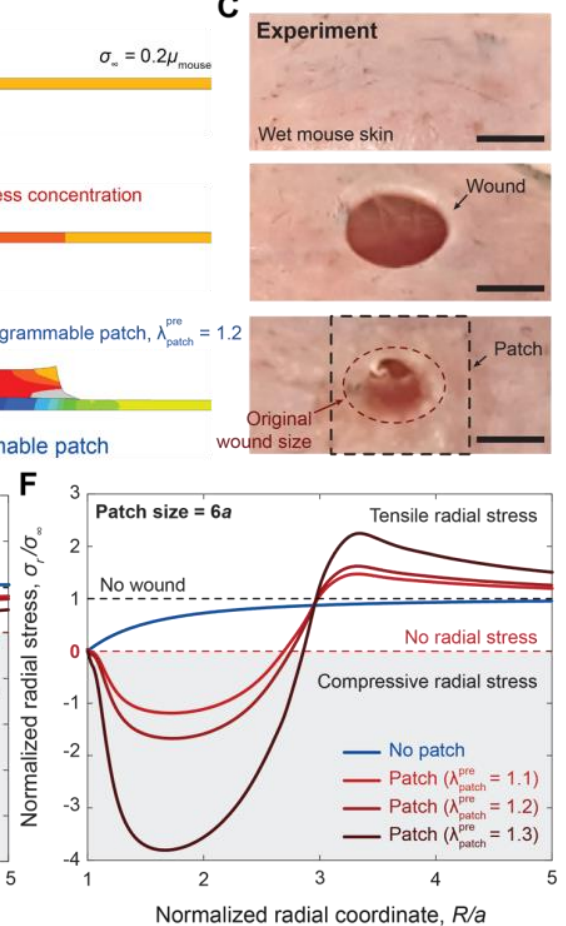

Fig. 4. Programmable closure and stress remodeling of diabetic mouse skin wounds. (A) Schematic illustration for the theoretical and finite-element analyses. (B and $\mathbf{C}$ ) Representative finite-element results (B) and the corresponding experiment images of the ex vivo diabetic mouse skin $(\mathrm{C})$ with $\lambda_{\text {patch }}^{\text {pre }}=1.3$ and patch diameter 3 times of the wound diameter. The shear modulus of the diabetic mice skin is denoted as $\mu_{\text {mouse }}$, the hoop stress in the diabetic mouse skin as $\sigma_{\theta}$, and the residual stress in the intact diabetic mouse skin as $\sigma_{\infty}$. (D) Finite-element and experimental results for the wound closure ratio as a function of $\lambda_{\text {patch }}^{\text {pre }}$ by varying the diameter of the strainprogrammable patch. FE, finite-element. ( $\mathbf{E}$ and $\mathbf{F})$ Finite-element results for the hoop $\sigma_{\theta}(\mathrm{E})$ and the radial $\sigma_{\mathrm{r}}(\mathrm{F})$ stresses around the wound with the strain-programmed patches with varying $\lambda_{\text {patch. }}^{\text {pre }}$ Scale bars, $5 \mathrm{~mm}(\mathrm{C})$. 
A

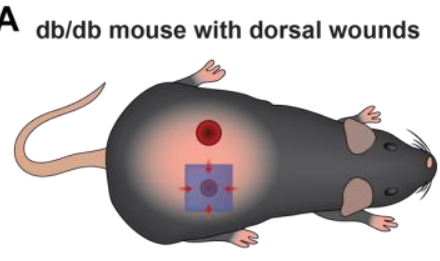

TD, no strain patch, patch with $\lambda_{\text {patch }}^{\text {pre }}=1.3$
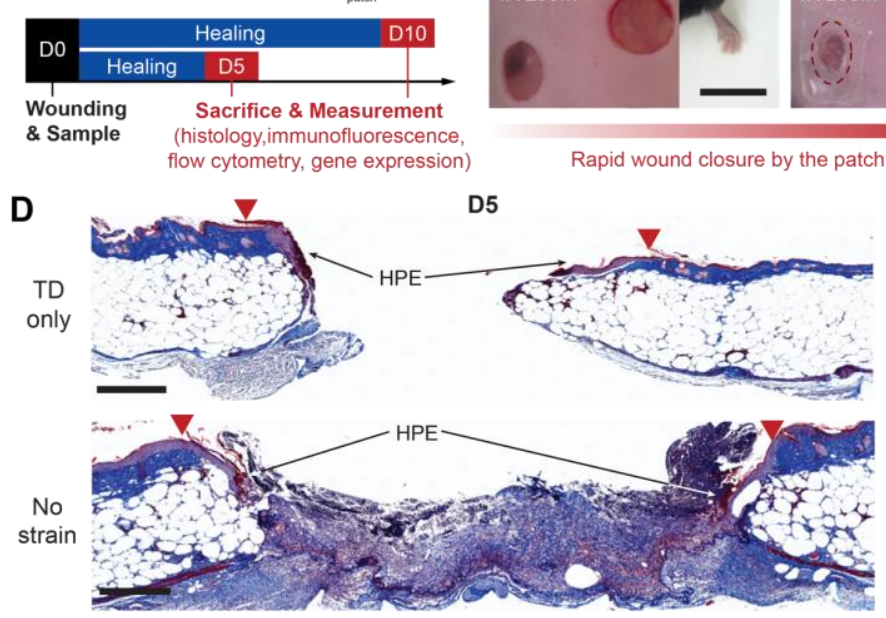

B
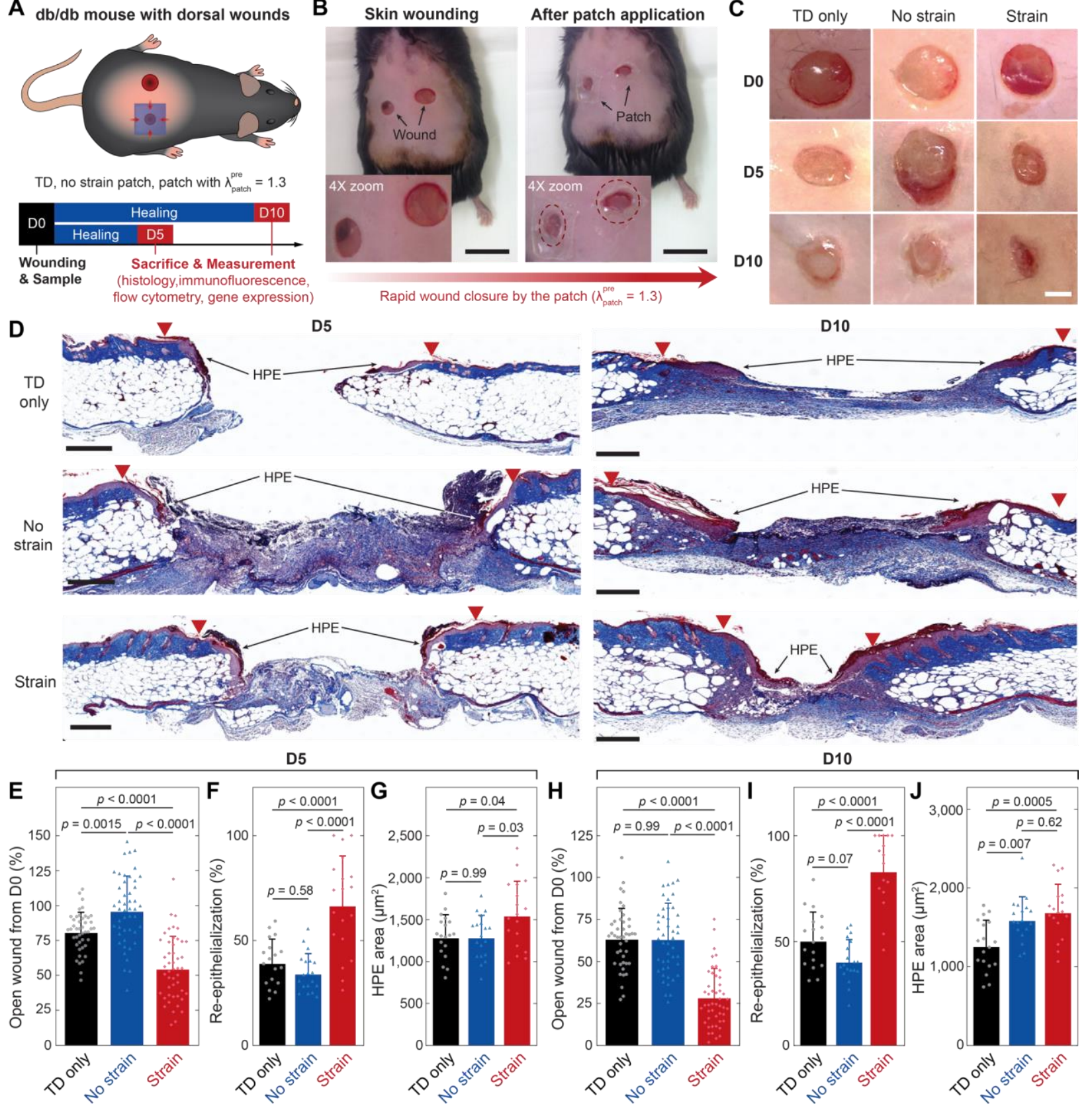

Fig. 5. Application of the strain-programmed patch in a mouse model of diabetic wound healing. (A) Schematic illustrations for the study design. (B) Representative images of $\mathrm{db} / \mathrm{db}$ mouse dorsal wounds before (left) and after (right) the strain-programmed patch application. (C) Representative macroscopic views of wounds on day 0 (D0), day 5 (D5), and day 10 (D10) for Tegaderm $^{\mathrm{TM}}(\mathrm{TD})$ only, no strain $\left(\lambda_{\text {patch }}^{\text {pre }}=1\right)$ and strain-programmed $\left(\lambda_{\text {patch }}^{\text {pre }}=1.3\right)$ patch experimental groups. The adhered patches were removed before imaging and measurement. (D) Representative images from D5 and D10 wounds with Masson's trichrome stain (MTS). Red triangles denote wound margins; HPE: hyperproliferative epidermis. (E-G) Quantification of the wound closure expressed as \% of open wound compared to D0 (E), the re-epithelialization expressed as \% with $100 \%$ being fully covered $(\mathrm{F})$, and the HPE area $(\mathrm{G})$ on D5. (H- 
bioRxiv preprint doi: https://doi.org/10.1101/2021.06.07.447423; this version posted June 7, 2021. The copyright holder for this preprint (which was not certified by peer review) is the author/funder, who has granted bioRxiv a license to display the preprint in perpetuity. It is made available under aCC-BY-NC-ND 4.0 International license.

J) Quantification of the wound closure expressed as \% of open wound compared to D0 (E), the reepithelialization expressed as \% (F), and the HPE area (G) on D10. Values in E-J represent the means $\pm \mathrm{SD}$ ( $n=16-48$ wounds). $P$ values were derived from one way ANOVA with Tukey's post-hoc tests. Scale bars, $20 \mathrm{~mm}(\mathrm{~B}) ; 3 \mathrm{~mm}$ (C); $500 \mu \mathrm{m}$ for D5 and $400 \mu \mathrm{m}$ for D10 (D). 

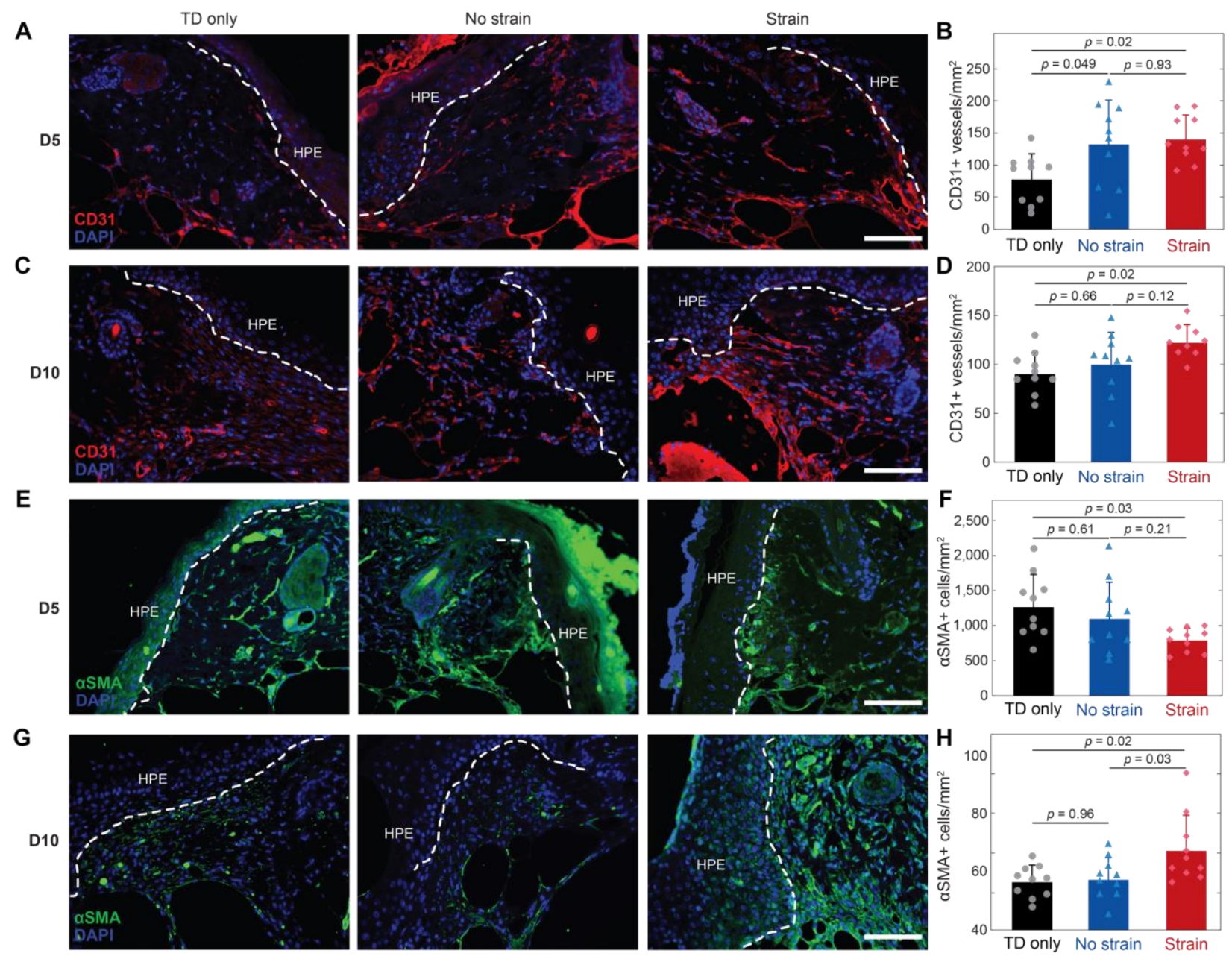

Fig. 6. Immunofluorescence staining analysis of diabetic mouse wounds. (A and B) Representative immunofluorescence images for CD31 (A) and quantification of CD31+ vessels per unit area (B) on day 5 (D5). (C and D) Representative immunofluorescence images for CD31 (C) and quantification of CD31+ vessels per unit area (D) on day 10 (D10). (E and F) Representative immunofluorescence images for $\alpha \mathrm{SMA}(\mathrm{E})$ and quantification of $\alpha \mathrm{SMA}+$ cells per unit area (F) on D5. (G and $\mathbf{H})$ Representative immunofluorescence images for $\alpha$ SMA (G) and quantification of $\alpha$ SMA + cells per unit area $(\mathrm{H})$ on D10. HPE: hyperproliferative epidermis. In immunofluorescence images, blue fluorescence corresponds to cell nuclei stained with 4',6diamidino-2-phenylindole (DAPI); red fluorescence corresponds to the expression on blood vessels (CD31); green fluorescence corresponds to the expression on fibroblast ( $\alpha \mathrm{SMA})$; dotted line represents the epidermis border. Experimental groups are Tegaderm ${ }^{\mathrm{TM}}$ (TD) only, no strain $\left(\lambda_{\text {patch }}^{\text {pre }}=1\right)$ and strain-programmed $\left(\lambda_{\text {patch }}^{\text {pre }}=1.3\right)$ patch for both D5 and D10. Values in B, D, F, $\mathrm{H}$ represent the means $\pm \mathrm{SD}$ ( $n=9-10$ wounds). $P$ values were derived from one way ANOVA with Tukey's post-hoc tests. Scale bars, $100 \mu \mathrm{m}(\mathrm{A}) ; 100 \mu \mathrm{m}(\mathrm{C}) ; 100 \mu \mathrm{m}(\mathrm{E}) ; 100 \mu \mathrm{m}(\mathrm{G})$. 


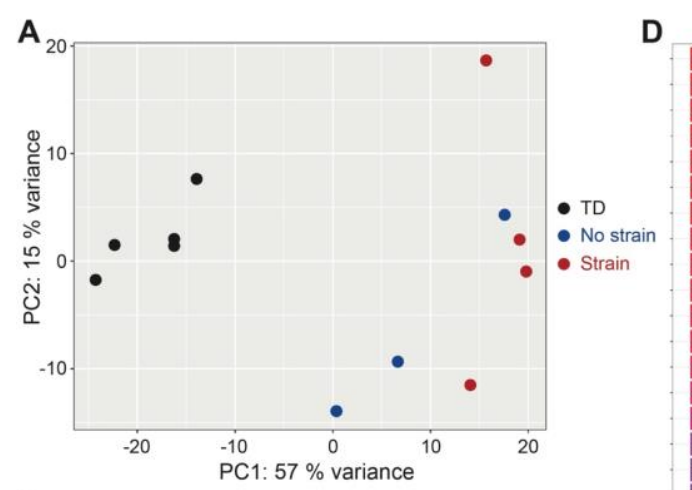

B Strain vs TD

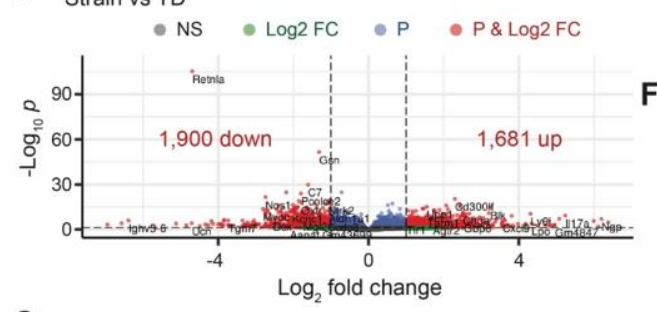

C Strain vs No strain
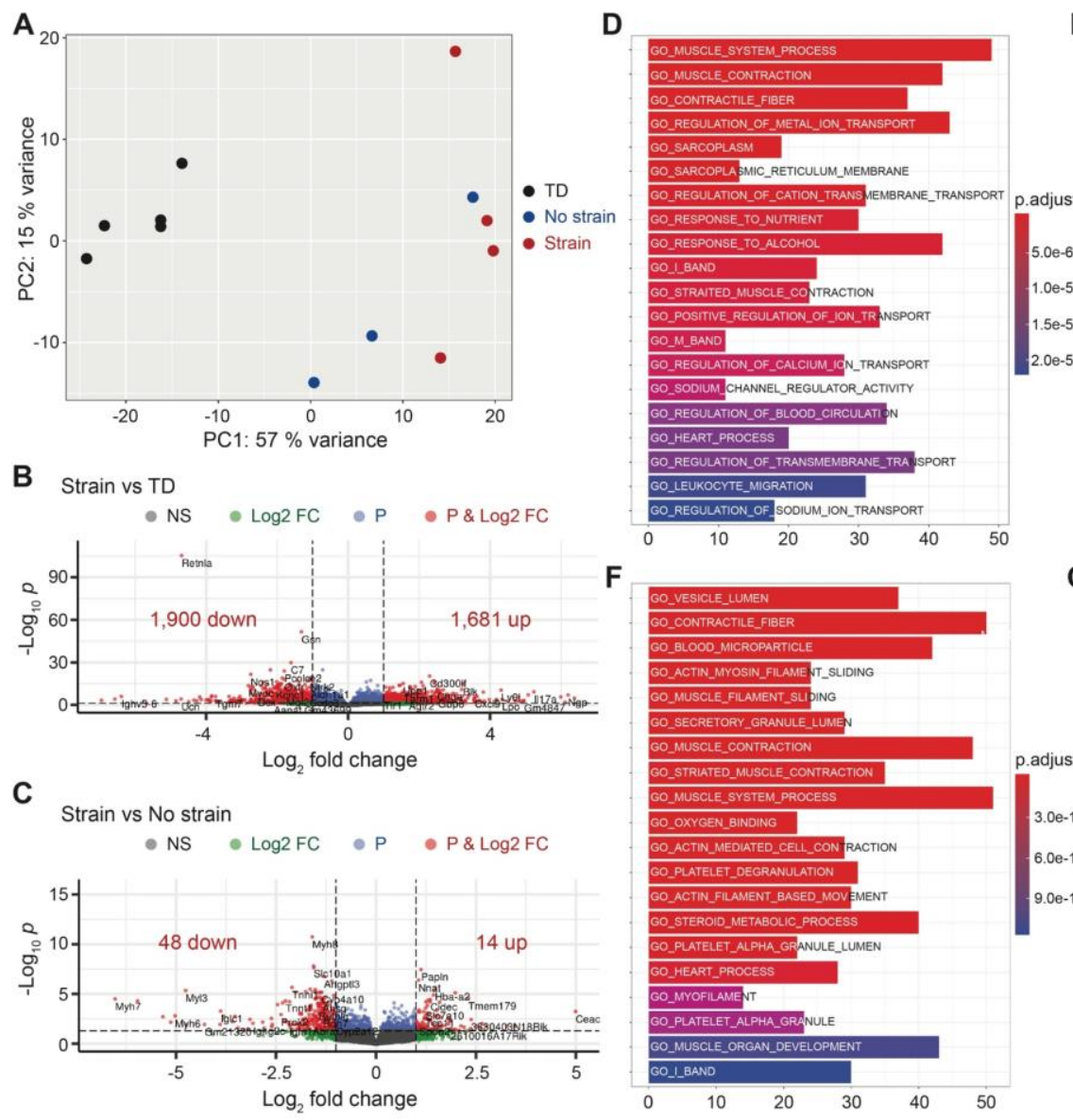

\section{E}

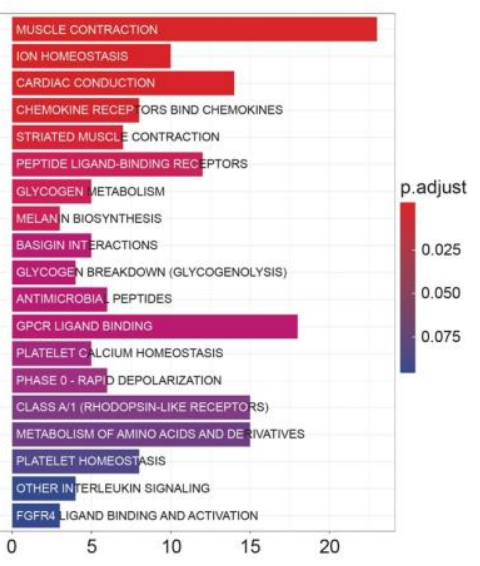

$\mathbf{F}$
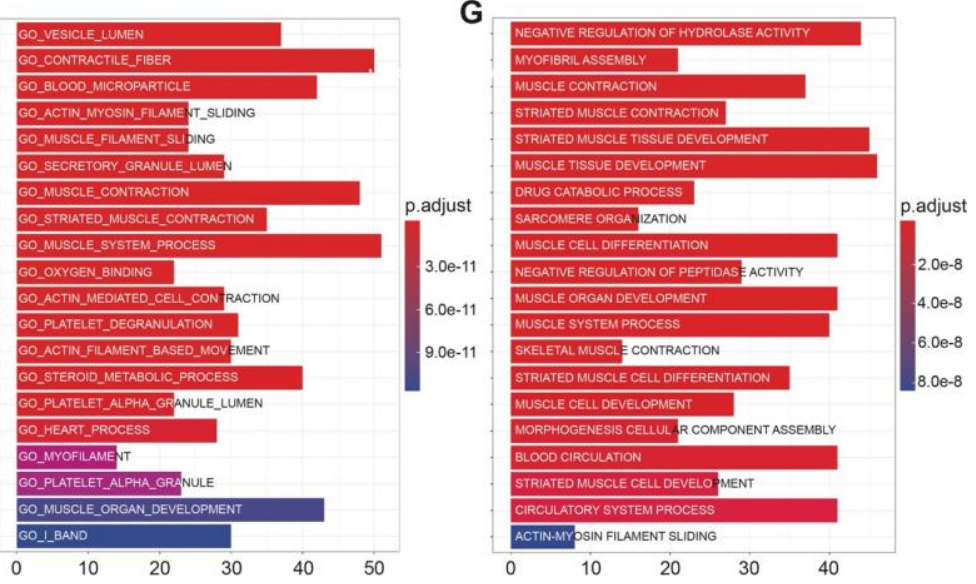

Fig. 7. Significantly altered global transcriptome of $\mathbf{d b} / \mathbf{d b}$ mouse skin wounds. (A) Principal component analysis (PCA) plot illustrating the variances of the Tegaderm ${ }^{\mathrm{TM}}$ (TD) only (black dots, $n=5)$, no strain $\left(\lambda_{\text {patch }}^{\text {pre }}=1\right)\left(\right.$ blue dots, $n=3$ ) and strain-programmed ( $\lambda_{\text {patch }}^{\text {pre }}=1.3$ ) (red dots, $n$ $=4)$ patch datasets. (B and $\mathbf{C})$ Volcano plots displaying gene expression profiles when comparing the strain-programmed patch against TD (B) and the strain-programmed against no strain (C) patches. Red colored data points represent genes that meet the thresholds of fold change (FC) above 1 or under -1 , False Discovery Rate (FDR) $<0.05$. (D to G) Functional over-representation analysis utilizing the top 500 differentially expressed genes results for Strain vs TD and Strain vs No strain in gene ontology (GO) (D and F) and Reactome (E and G) databases. The $\mathrm{x}$-axis corresponds to the number of genes implicated in each pathway and the color of the bars correlates with the adjusted $p$-values as shown in the legends. 


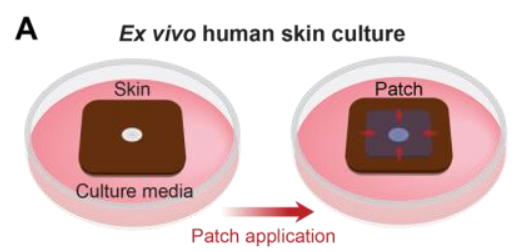

No strain \& strain-programmed patch with $\lambda_{\text {patch }}^{\text {pre }}=1.3$

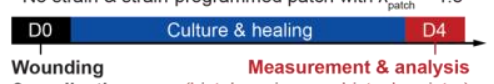

$\begin{array}{lr}\text { Wounding } & \text { Measurement \& analysis } \\ \text { \& application } & \text { (histology, immunohistochemistry) }\end{array}$

C

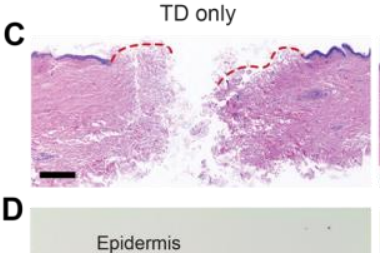

$$
\text { Epidermis }
$$

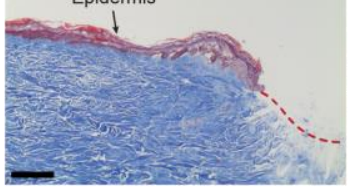

Epidermis

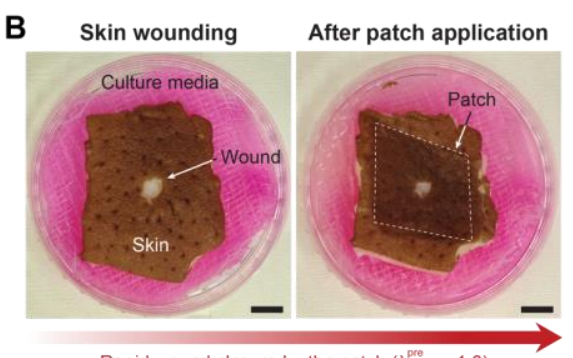

Rapid wound closure by the patch $\left(\lambda_{\text {patch }}^{\text {pre }}=1.3\right)$

No strain
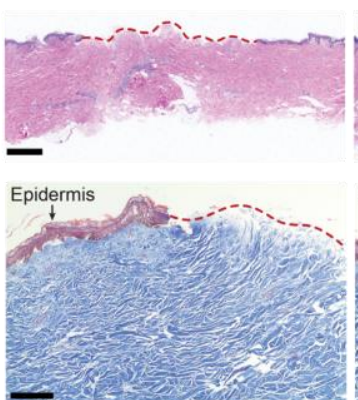

Strain

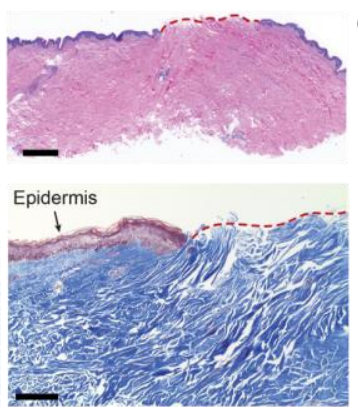

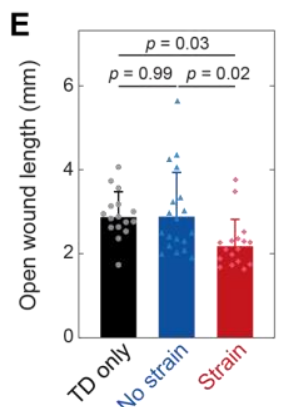
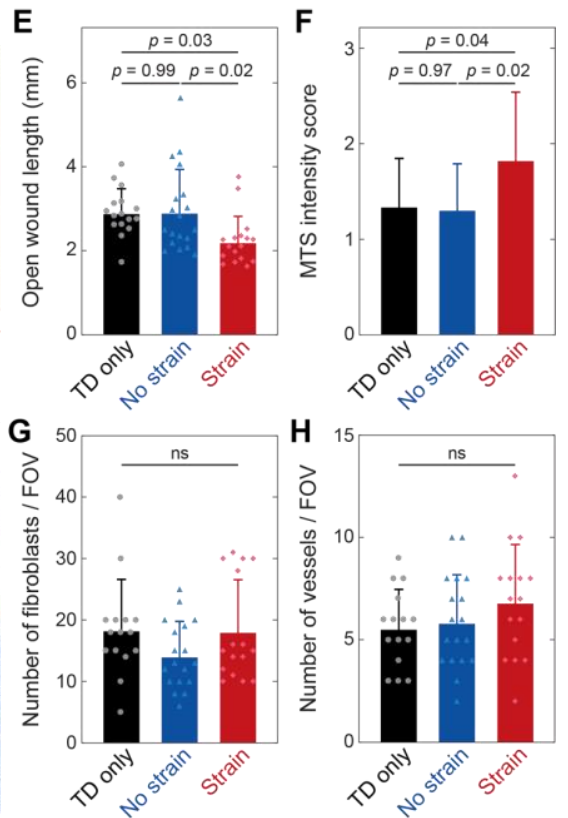

Fig. 8. Application of the strain-programmed patch in a human ex vivo model of wound healing. (A) Schematic illustrations for the study design. (B) Representative images of an ex vivo human skin culture setup before (left) and after (right) the strain-programmed patch application. (C and D) Representative images from day 4 (D4) wounds for Tegaderm ${ }^{\mathrm{TM}}$ (TD) only, no strain $\left(\lambda_{\text {patch }}^{\text {pre }}=1\right)$ and strain-programmed $\left(\lambda_{\text {patch }}^{\text {pre }}=1.3\right)$ patch experimental groups with hematoxylin and eosin stain (H\&E) (C) and Masson's trichrome stain (MTS) (D). Red dotted line marks the non-epithelized tissue. (E-H) Quantification of the open wound length (E), the MTS intensity score (F), the number of fibroblast-like cells per field of view (FOV) (G), and the number of vessels per FOV $(\mathrm{H})$ on D4. Values in E-H represent the means \pm SD $(n=15-19$ wounds from 3 individual patients' skin). $P$ values were derived from one way ANOVA with Tukey's post-hoc tests. Scale bars, $10 \mathrm{~mm}$ (B); $800 \mu \mathrm{m}$ (C); $200 \mu \mathrm{m}(\mathrm{D})$. 


\section{SUPPLEMENTARY MATERIALS}

\section{Supplementary Texts}

\section{Hydration-based shape-memory of the strain-programmable patch}

To achieve programmable contraction and stress remodeling of wet wounded tissues, we develop a hydration-based shape-memory mechanism and an associated theoretical framework for predictable and reproducible fabrication for the strain-programmable patch. In the following paragraphs, we will discuss (i) a physical picture of the proposed hydration-based shape-memory mechanism, (ii) fabrication of the strain-programmable patch, and (iii) mechanical properties of the strain-programmable patch.

\section{Hydration-based shape-memory mechanism}

Shape-memory polymers are the class of polymers capable of memorizing a certain macroscopic deformed configuration in the stable fixed state and relaxing to the original undeformed configuration under external stimuli $(28,29,82)$. The transition from the fixed deformed configuration to the relaxed original configuration relies on a drastic mechanical property changes between the glassy fixed state and the rubbery relaxed state $(28,29,82)$. In the glassy state, polymer chains in the shape-memory polymers are "frozen" with suppressed mobility and high macroscopic stiffness (i.e., high Young's modulus). In the rubbery state, polymer chains in the shape-memory polymers recover their entropic elasticity with low macroscopic stiffness (i.e., low Young's modulus) where the memorized deformation in the fixed glassy state can be elastically relaxed. The glassy to rubbery state transition in shape-memory polymers are conventionally achieved by tuning environmental temperature across the transition temperature $\left(T_{\text {trans }}\right)$ where the shape-memory polymers are in the glassy state in low temperature $\left(T<T_{\text {trans }}\right)$ and in the rubbery state in high temperature $\left(T>T_{\text {trans }}\right.$ ), respectively $(27-29,82) . T_{\text {trans }}$ is a critical temperature where the chain mobility of the shape-memory polymers changes drastically, which can be either the glass transition temperature $\left(T_{\mathrm{g}}\right)$ for amorphous polymers or the melting temperature $\left(T_{\mathrm{m}}\right)$ for crystalline polymers.

In this work, we propose a non-thermal hydration-based shape-memory mechanism without the need of changing environmental temperature to achieve highly programmable and facile contraction and mechanical modulations of wet wounded tissues in synergistic combination with the dry-crosslinking mechanism for rapid robust wet adhesion $(23,26)$. The hydration-based shape-memory mechanism relies on the hydrogels' unique transition between the glassy state and the rubbery state based on hydration level. In hydrated or swollen state, hydrogels are elastically deformable solids in the rubbery state with low macroscopic stiffness (i.e., low Young's modulus). However, when hydrogels are dried to remove water, hydrogels transit into the glassy state whose polymer chains are frozen with suppressed mobility and high macroscopic stiffness (i.e., high Young's modulus), providing ability to stably fix or memorize the deformed configuration and program the applied pre-stretches. By re-hydrating the dried hydrogels, hydrogels can recover their soft and elastic properties in the rubbery state during which the programmed pre-stretches can be elastically relaxed (Fig. 1A).

This hydration-based transition between the glassy and rubber states is due to the reduction of polymer network's $T_{\mathrm{g}}$ during hydration. A simplified expression for $T_{\mathrm{g}}$ is provided as $(24,25)$ 


$$
T_{\mathrm{g}}=\frac{\alpha_{1} \phi_{1} T_{\mathrm{g} 1}+\alpha_{2} \phi_{2} T_{\mathrm{g} 2}}{\alpha_{1} \phi_{1}+\alpha_{2} \phi_{2}}
$$

where $\alpha_{1}$ and $\alpha_{2}$ are the thermal expansion coefficients of the polymer and the solvent, and $\phi_{1}$ and $\phi_{2}$ are the volume fraction of the polymer and solvent, $T_{\mathrm{g} 1}$ and $T_{\mathrm{g} 2}$ are the glass transition temperature of the polymer and the melting point of the solvent, respectively. The melting point $T_{\mathrm{g} 2}$ of water is much lower than the glass transition temperature $T_{\mathrm{g} 1}$ of typical polymers for hydrogels. Notably, the time required to fully hydrate a hydrogel with a thickness of $H$ can be expressed as $t_{\text {hydrate }}=\left(H / k_{\mathrm{h}}\right)^{2}$ where $k_{\mathrm{h}}$ is the hydration coefficient of the hydrogel (26).

Hence, the proposed hydration-based shape-memory mechanism provides a facile and effective strain-programming and -release strategy for hydrogels, including the bioadhesive layer of the strain-programmable patch, only by natively present water in wet physiological environment without the need of thermal or other complex external stimuli. The hydration-based shape-memory mechanism's highly versatile and tunable strain-programming capability and water-based biocompatible triggering of the programmed strain release will be particularly advantageous for biomedical and clinical applications operating in wet physiological environments.

\section{Fabrication of the strain-programmable patch}

Taking advantage of the hydration-based shape-memory mechanism, the strain-programmable patch can be fabricated based on multiple steps of pre-stretch and drying processes as summarized in fig. S3. Since the non-adhesive elastomer backing and the bioadhesive layer in the strainprogrammable patch can have different swelling ratio in wet physiological environments (fig. S4), the fabrication process of the strain-programmable patch involves canceling of swelling mismatch between the two layers as well as swelling of the assembled patch.

The fabrication of the strain-programmable patch starts from the as-prepared bioadhesive in the rubbery state with the dimension of $\lambda_{\text {adhesive }}^{0}(=1.48$ for the bioadhesive used in this work) in length and width and $H_{\text {adhesive }} \lambda_{\text {adhesive }}^{0}$ in thickness where the reference state (i.e., isotropically dried bioadhesive) has the dimension of $H_{\text {adhesive }}$ in thickness and unity in length and width. The fabrication process consists of five distinctive steps as the following:

Step 1. Introduce the non-adhesive elastomer backing resin (i.e., before curing) on the as-prepared bioadhesive layer, and pre-stretch the bioadhesive layer by ratio of

$$
\lambda_{\text {adhesive }}^{\text {pre }}=\frac{\lambda_{\text {adhesive }}^{\infty}}{\lambda_{\text {adhesive }}^{0} \lambda_{\text {backing }}^{\infty}}
$$

in both directions to cancel out the swelling mismatch between the non-adhesive elastomer backing and the bioadhesive layer (Step 1 in fig. S3), where $\lambda_{\text {adhesive }}^{\infty}$ is the equilibrium swelling ratio of the bioadhesive layer and $\lambda_{\text {backing }}^{\infty}$ is the equilibrium swelling ratio of the non-adhesive elastomer backing. For the bioadhesive and the elastomer backing used in this work, $\lambda_{\text {adhesive }}^{\infty}=3.46$ and $\lambda_{\text {backing }}^{\infty}=1.4$ which give $\lambda_{\text {adhesive }}^{\text {pre }}=1.665$ (fig. S4). 
After this step, the bioadhesive layer in the rubbery state has the dimension of $\lambda_{\text {adhesive }}^{0} \lambda_{\text {adhesive }}^{\text {pre }}$ in length and width and $\left(H_{\text {adhesive }} \lambda_{\text {adhesive }}^{0}\right)\left(\lambda_{\text {adhesive }}^{\text {pre }}\right)^{-2}$ in thickness.

Step 2. Cure the non-adhesive elastomer backing on the pre-stretched bioadhesive layer (Step 2 in fig. S3).

After this step, the non-adhesive elastomer backing in the rubbery state has the dimension of $\lambda_{\text {adhesive }}^{0} \lambda_{\text {adhesive }}^{\text {pre }}$ in length and width and $H_{\text {backing }}$ in thickness; the bioadhesive layer in the rubbery state has the dimension of $\lambda_{\text {adhesive }}^{0} \lambda_{\text {adhesive }}^{\text {pre }}$ in length and width and $\left(H_{\text {adhesive }} \lambda_{\text {adhesive }}^{0}\right)\left(\lambda_{\text {adhesive }}^{\text {pre }}\right)^{-2}$ in thickness.

Step 3. Pre-stretch both non-adhesive elastomer backing and bioadhesive layer by ratio of

$$
\lambda_{\text {backing }}^{\text {pre }}=\lambda_{\text {backing }}^{\infty}
$$

in both directions to cancel out the dimensional change of the patch by swelling in wet physiological environments (Step 3 in fig. S3).

After this step, the non-adhesive elastomer backing in the rubbery state has the dimension of $\lambda_{\text {adhesive }}^{0} \lambda_{\text {adhesive }}^{\text {pre }} \lambda_{\text {backing }}^{\text {pre }}$ in length and width and $\left(H_{\text {backing }}\right)\left(\lambda_{\text {backing }}^{\text {pre }}\right)^{-2}$ in thickness; the bioadhesive layer in the rubbery state has the dimension of $\lambda_{\text {adhesive }}^{0} \lambda_{\text {adhesive }}^{\text {pre }} \lambda_{\text {backing }}^{\text {pre }}$ in length and width and $\left(H_{\text {adhesive }} \lambda_{\text {adhesive }}^{0}\right)\left(\lambda_{\text {adhesive }}^{\text {pre }} \lambda_{\text {backing }}^{\text {pre }}\right)^{-2}$ in thickness.

Step 4. Pre-stretch both non-adhesive elastomer backing and bioadhesive layer by ratios of $\lambda_{\text {patch }}^{\text {pre } 1}$ and $\lambda_{\text {patch }}^{\text {pre } 2}$ to each direction to program desired strains (direction 1 in length, direction 2 in width), respectively (Step 4 in fig. S3).

After this step, the non-adhesive elastomer backing in the rubbery state has the dimension of $\lambda_{\text {adhesive }}^{0} \lambda_{\text {adhesive }}^{\text {pre }} \lambda_{\text {backing }}^{\text {pre }} \lambda_{\text {patch }}^{\text {pre } 1}$ in length, $\lambda_{\text {adhesive }}^{0} \lambda_{\text {adhesive }}^{\text {pre }} \lambda_{\text {backing }}^{\text {pre }} \lambda_{\text {patch }}^{\text {pre } 2}$ in width, and ( $\left.H_{\text {backing }}\right)\left(\left(\lambda_{\text {backing }}^{\text {pre }}\right)^{2} \lambda_{\text {patch }}^{\text {pre1 }} \lambda_{\text {patch }}^{\text {pre2 }}\right)^{-1}$ in thickness; the bioadhesive layer in the rubbery state has the dimension of $\lambda_{\text {adhesive }}^{0} \lambda_{\text {adhesive }}^{\text {pre }} \lambda_{\text {backing }}^{\text {pre }} \lambda_{\text {patch }}^{\text {pre } 1}$ in length, $\lambda_{\text {adhesive }}^{0} \lambda_{\text {adhesive }}^{\text {pre }} \lambda_{\text {backing }}^{\text {pre }} \lambda_{\text {patch }}^{\text {pre } 2}$ in width, and $\left(H_{\text {adhesive }} \lambda_{\text {adhesive }}^{0}\right)\left(\left(\lambda_{\text {adhesive }}^{\text {pre }} \lambda_{\text {backing }}^{\text {pre }}\right)^{2} \lambda_{\text {patch }}^{\text {pre1 }} \lambda_{\text {patch }}^{\text {pre2 }}\right)^{-1}$ in thickness.

Step 5. Dry the assembled strain-programmable patch to shape-memory the pre-stretched configuration (Step 5 in fig. S3), completing the fabrication of the strain-programmable patch.

After this step, the non-adhesive elastomer backing in the rubbery state has the dimension

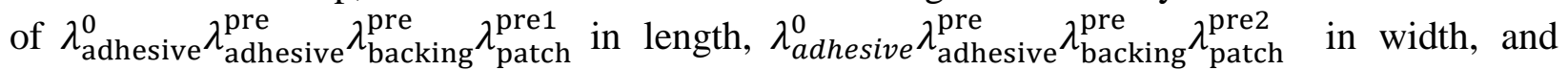
( $\left.H_{\text {backing }}\right)\left(\left(\lambda_{\text {backing }}^{\text {pre }}\right)^{2} \lambda_{\text {patch }}^{\text {pre1 }} \lambda_{\text {patch }}^{\text {pre2 }}\right)^{-1}$ in thickness; the bioadhesive layer in the glassy state has 


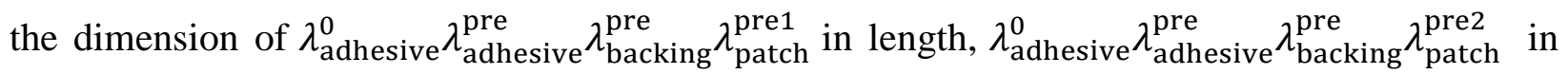
width, and $\left(H_{\text {adhesive }}\right)\left(\left(\lambda_{\text {adhesive }}^{0} \lambda_{\text {adhesive }}^{\text {pre }} \lambda_{\text {backing }}^{\text {pre }}\right)^{2} \lambda_{\text {patch }}^{\text {pre1 }} \lambda_{\text {patch }}^{\text {pre2 }}\right)^{-1}$ in thickness.

\section{Mechanical properties of the strain-programmable patch}

\subsection{Measurement of physical parameters of the strain-programmable patch}

Since both non-adhesive elastomer backing and bioadhesive layer of the strain-programmable patch used in this work become hydrogel in wet physiological environments, we take the swollen strain-programmable patch as a Flory-Rehner hydrogel with thermodynamic parameters of $N_{\text {adhesive }}, N_{\text {backing }}, \chi_{\text {adhesive }}$, and $\chi_{\text {backing }}$ for simplicity of the analysis, where $N_{\text {adhesive }}$ is the number of polymer chains per unit volume of the bioadhesive at the reference state (fig. S3), $N_{\text {backing }}$ is the number of polymer chains per unit volume of the elastomer backing at the reference state (fig. S3), $\chi$ adhesive is the Flory solvent-polymer interaction parameter for the bioadhesive, and $\chi_{\text {backing }}$ is the Flory solvent-polymer interaction parameter for the elastomer backing $(83,84)$.

To determine $N_{\text {adhesive }}$, the shear modulus $\mu_{\text {adhesive }}^{0}$ is measured for the as-prepared bioadhesive and $N_{\text {adhesive }}$ is then calculated from (85)

$$
\mu_{\text {adhesive }}^{0}=\frac{1}{\lambda_{\text {adhesive }}^{0}} N_{\text {adhesive }} k T
$$

where $k$ is the Boltzmann constant, $T$ is the absolute temperature. To measure $\chi$ adhesive, we carry out free-swelling experiment for the as-prepared bioadhesive without constraint (i.e., freely swelling in PBS). The Cauchy stress $\sigma_{\text {eq,adhesive }}$ in any direction of the unconstrained equilibrium swollen bioadhesive can be expressed as (85)

$$
\begin{gathered}
\sigma_{\text {eq,adhesive }}=N_{\text {adhesive }} k T\left(\lambda_{\text {adhesive }}^{\infty}-\lambda_{\text {adhesive }}^{\infty}\right) \\
+\frac{k T}{v}\left[\ln \left(1-\lambda_{\text {adhesive }}^{\infty}\right)+\lambda_{\text {adhesive }}^{\infty}+\chi_{\text {adhesive }} \lambda_{\text {adhesive }}^{\infty}\right]
\end{gathered}
$$

where $v$ is the volume of the water molecule. Due to the traction-free boundary condition, the Cauchy stress $\sigma_{\text {eq,adhesive }}$ is zero in Eq. S5. In this study, we used $T=310 \mathrm{~K}$ (i.e., body temperature), $v=3.0 \times 10^{-29} \mathrm{~m}^{3}$ and experimentally-measured values of $\mu_{\text {adhesive }}^{0}=45 \mathrm{kPa}$, $\lambda_{\text {adhesive }}^{0}=1.48$, and $\lambda_{\text {adhesive }}^{\infty}=3.46$. By implementing these values and solving Eq. S5, we can calculate $N_{\text {adhesive }}=1.56 \times 10^{25} \mathrm{~m}^{-3}$ and $\chi_{\text {adhesive }}=0.29$.

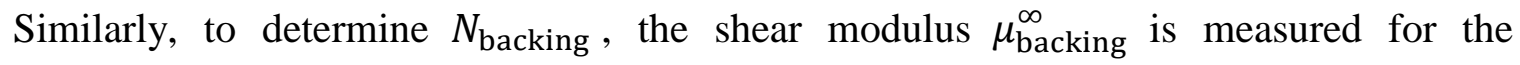
equilibrium swollen elastomer backing and $N_{\text {backing }}$ is then calculated from (85)

$$
\mu_{\text {backing }}^{\infty}=\frac{1}{\lambda_{\text {backing }}^{\infty}} N_{\text {backing }} k T
$$


To measure $\chi_{\text {backing, }}$, we carry out free-swelling experiment for the elastomer backing without

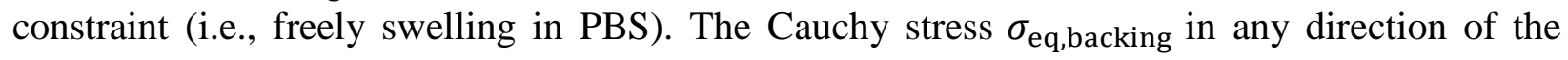
unconstrained equilibrium swollen elastomer backing can be expressed as (85)

$$
\begin{gathered}
\sigma_{\text {eq,backing }}=N_{\text {backing }} k T\left(\lambda_{\text {backing }^{\infty}}{ }^{-1}-\lambda_{\text {backing }}^{\infty}{ }^{-3}\right) \\
+\frac{k T}{v}\left[\ln \left(1-\lambda_{\text {backing }}^{\infty}\right)+\lambda_{\text {backing }}^{\infty}+\chi_{\text {backing }}^{\infty} \lambda_{\text {backing }}^{\infty}\right]
\end{gathered}
$$

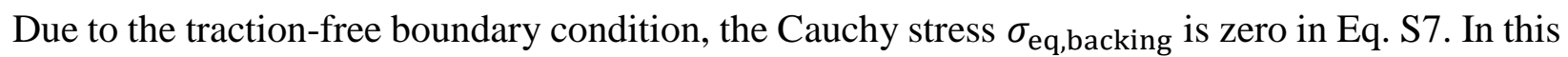
study, we used $T=310 \mathrm{~K}$ (i.e., body temperature), $v=3.0 \times 10^{-29} \mathrm{~m}^{3}$ and experimentallymeasured values of $G_{\text {backing }}^{\infty}=720 \mathrm{kPa}$ and $\lambda_{\text {backing }}^{\infty}=1.4$. By implementing these values and solving Eq. S7, we can calculate $N_{\text {backing }}=2.36 \times 10^{26} \mathrm{~m}^{3}$ and $\chi_{\text {backing }}=0.65$.

\subsection{Flory-Rehner model of the strain-programmable patch}

In the equilibrium swollen state, the Cauchy stresses (or true stresses) generated by the bioadhesive layer $\sigma_{\text {adhesive }}$ and the elastomer backing $\sigma_{\text {backing }}$ can be expressed as $(85,86)$

$$
\begin{aligned}
\sigma_{\text {adhesive }, \mathrm{i}} & =\frac{N_{\text {adhesive }} k T}{J_{\text {adhesive }}}\left(\lambda_{\text {adhesive }, \mathrm{i}}^{2}-1\right)+\frac{k T}{v}\left(\ln \frac{J_{\text {adhesive }}-1}{J_{\text {adhesive }}}+\frac{1}{J_{\text {adhesive }}}+\frac{\chi_{\text {adhesive }}}{J_{\text {adhesive }}{ }^{2}}\right) \\
\sigma_{\text {backing,i }} & =\frac{N_{\text {backing }} k T}{J_{\text {backing }}}\left(\lambda_{\text {backing,i }}^{2}-1\right)+\frac{k T}{v}\left(\ln \frac{J_{\text {backing }}-1}{J_{\text {backing }}}+\frac{1}{J_{\text {backing }}}+\frac{\chi_{\text {backing }}}{J_{\text {backing }}{ }^{2}}\right)
\end{aligned}
$$

where $\sigma_{\mathrm{i}}$ is the Cauchy stress for each principal direction ( $\mathrm{i}=1$ for length, 2 for width, and 3 for thickness direction, respectively), $\lambda_{\mathrm{i}}$ is the stretch from the reference state for each principal direction ( $i=1$ for length, 2 for width, and 3 for thickness direction, respectively), $J_{\text {adhesive }}=$ $\lambda_{\text {adhesive }, 1} \lambda_{\text {adhesive, } 2} \lambda_{\text {adhesive, } 3}$, and $J_{\text {backing }}=\lambda_{\text {backing, } 1} \lambda_{\text {backing, } 2} \lambda_{\text {backing, } 3}$.

Since the strain-programmable patch consists of the elastomer backing and the bioadhesive layer, the overall Cauchy stress generated by the strain-programable patch in equilibrium swollen state can be expressed as

$$
\sigma_{\text {patch,i }}=\gamma_{\text {backing }} \sigma_{\text {backing,i }}+\left(1-\gamma_{\text {backing }}\right) \sigma_{\text {adhesive, } \mathrm{i}}
$$

where $\gamma_{\text {backing }}$ is the ratio of the elastomer backing thickness in the total thickness of the equilibrium swollen strain-programmable patch which can be calculated as

$$
\gamma_{\text {backing }}=\frac{H_{\text {backing }} \lambda_{\text {backing }}^{\infty}}{H_{\text {adhesive }} \lambda_{\text {adhesive }}^{\infty}+H_{\text {backing }} \lambda_{\text {backing }}^{\infty}}
$$

where $H_{\text {backing }}$ and $H_{\text {adhesive }}$ are thicknesses of the elastomer backing and the bioadhesive at the reference state, respectively (fig. S3).

\subsection{Neo-Hookean model approximation of the strain-programmable patch}


While we model the strain-programmable patch in equilibrium swollen state as a Flory-Rehner hydrogel, we also investigate approximation of the strain-programmable patch as an incompressible neo-Hookean solid (87) for simplicity of the analysis, particularly in finite-element modeling. To obtain shear moduli of materials for incompressible neo-Hookean models, we first fit the uniaxial tensile test data of the elastomer backing, the bioadhesive layer, and the strainprogrammable patch in equilibrium swollen state by using the following expression

$$
S=\mu\left(\lambda-\frac{1}{\lambda^{2}}\right)
$$

where $S$ is the nominal or engineering stress, $\mu$ is the shear modulus, and $\lambda$ is the stretch of sample in tensile tests. As a result, we obtain shear moduli of the elastomer backing $\mu_{\text {backing }}^{\infty}=720 \mathrm{kPa}$, the bioadhesive layer $\mu_{\text {adhesive }}^{\infty}=13 \mathrm{kPa}$, and the strain-programmable patch $\mu_{\mathrm{patch}}^{\infty}=17.2 \mathrm{kPa}$ in equilibrium swollen state, respecitvely (Fig. 2D and fig. S2).

As illustrated in fig. S5, the Flory-Rehner model and the incompressible neo-Hookean model show good agreement for the equilibrium swollen strain-programmable patch. This indicates that the simpler incompressible neo-Hookean model can be used to describe the mechanical behavior of the strain-programmable patch. 


\section{Modeling of wound contraction and stress remodeling by the strain-programmable patch}

To provide quantitative and predictive design guideline for contraction and stress remodeling of wounds by the strain-programmable patch, we develop analytical and finite-element models based on mechanical properties of the strain-programmable patch and tissues. In this study, without loss of generality, our models are developed based on a circular wound in the skin which is a common form for diabetic wounds and an equibiaxially strain-programmed patch (i.e., $\lambda_{\text {patch }}^{\text {pre }}=\lambda_{\text {patch }}^{\text {pre }}=$ $\lambda_{\text {patch }}^{\text {pre }}$.

\section{Analytical modeling}

\subsection{Initial enlarging of wound by pre-strain in native skin}

A skin wound with the initial radius denoted as $a$ enlarges to $a_{1}$ immediately after wounding due to the relaxation of the pretension $\sigma_{\infty}$ around the wound edge generated by the pre-strain in native skin. The reference state refers to an imaginary stress-free state in which $a_{0}=a\left(\lambda_{\text {skin }}^{\text {pre }}\right)^{-1}$ where $\lambda_{\text {skin }}^{\text {pre }}=1.035$ is the experimentally measured pre-strain within the native skin. When the strain-

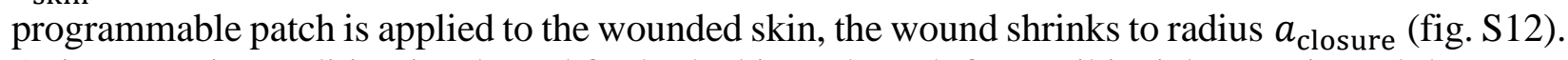
Axisymmetric condition is adopted for both skin and patch for equibiaxial scenario and the outer boundary of skin is assumed to be much larger than the wound (i.e., $\left.c_{0}=c\left(\lambda_{\text {skin }}^{\text {pre }}\right)^{-1} \gg a_{0}\right)$.

The strain-programmable patch initially has a radius of $b_{0}$ and undergoes an equibiaxial stretch of $\lambda_{\text {patch }}^{\text {pre }}$. For an incompressible neo-Hookean material under equibiaxial stretch, the Cauchy stresses are calculated as

$$
\sigma_{r, \text { patch }}=\sigma_{\theta, \text { patch }}=\mu_{\text {patch }}^{\infty}\left(\lambda_{\text {patch }}^{\text {pre }}{ }^{2}-\frac{1}{\lambda_{\text {patch }}^{\text {pre }}}\right)
$$

where $\mu_{\text {patch }}^{\infty}$ is the shear modulus of the strain-programmable patch in equilibrium swollen state. The skin is modeled as an incompressible Ogden material and the pretension in the skin before wounding can be found as

$$
\sigma_{\infty}=\frac{2 \mu_{\text {skin }}}{\alpha}\left(\lambda_{\text {skin }}^{\text {pre } \alpha}-\frac{1}{\lambda_{\text {skin }}^{\text {pre } 2 \alpha}}\right)
$$

where $\mu_{\text {skin }}=40 \mathrm{kPa}$ and $\alpha=20$ (for human skin) are fitting parameters measured from experiment. By plugging $\lambda_{\text {skin }}^{\text {pre }}=1.035$ into Eq. S13, one can calculate that $\sigma_{\infty}=0.17 \mu_{\text {skin }}$.

Next, we can solve the wound radius $a_{1}$ immediately after wounding of the skin. Consider an arbitrary circle with radius $R \geq a_{0}$ in the reference state and moves to $r$ in the deformed configuration. The incompressibility requires

$$
\lambda_{z}\left(r^{2}-a_{1}^{2}\right)=R^{2}-a_{0}^{2}
$$

where $\lambda_{z}$ is the stretch in z-direction. Then 


$$
r=a_{0} \lambda_{\theta} \sqrt{\frac{\lambda_{\theta \mid a_{0}}^{2} \lambda_{z}-1}{\lambda_{\theta}^{2} \lambda_{z}-1}}
$$

where hoop stretch $\lambda_{\theta}=r / R$ and $\lambda_{\theta \mid a_{0}}=a_{1} / a_{0}$ is the hoop stretch at the wound boundary $R=$ $a_{0}$ and $\lambda_{r} \lambda_{\theta} \lambda_{z}=1$. The equilibrium equation in current configuration can be expressed as

$$
\frac{\partial \sigma_{r}}{\partial r}+\frac{\sigma_{r}-\sigma_{\theta}}{r}=0
$$

To solve this, we can change variable from $r$ to $\lambda_{\theta}$. With Eq. S16, we have

$$
\frac{\partial \sigma_{r}}{\partial r} d r=-\frac{\sigma_{r}-\sigma_{\theta}}{r} d r=-\frac{2 \mu_{\text {skin }}}{\alpha \lambda_{\theta}}\left(\frac{1}{\lambda_{z} \lambda_{\theta}}\right)^{\alpha} \frac{1-\lambda_{z}^{\alpha} \lambda_{\theta}^{2 \alpha}}{1-\lambda_{z} \lambda_{\theta}^{2}} d \lambda_{\theta}
$$

By integrating Eq. S17 from $r=a$ to arbitrary $r>a_{1}$, we obtain

$$
\sigma_{r r}-\left.\sigma_{r r}\right|_{r=a_{1}}=\phi\left(\lambda_{z}, \lambda_{\theta}\right)-\phi\left(\lambda_{z}, \lambda_{\theta \mid a_{0}}\right)
$$

where $\phi\left(\lambda_{z}, \lambda_{\theta}\right)$ is analytical function of $\lambda_{z}, \lambda_{\theta}$ and $\lambda_{\theta \mid a_{0}}$ is the hoop stretch at $r=a_{0}$. Due to the lengthy expression, we do not provide the complete form of $\phi\left(\lambda_{z}, \lambda_{\theta}\right)$ here. By invoking the boundary conditions at $r=a_{1},\left.\sigma_{r r}\right|_{r=a_{1}}=0$, and at $r=c_{1},\left.\sigma_{r r}\right|_{r=c_{1}}=\sigma_{\infty}$, one can have

$$
\sigma_{\infty}=\phi\left(\lambda_{z}, \lambda_{\theta \mid c_{0}}\right)-\phi\left(\lambda_{z}, \lambda_{\theta \mid a_{0}}\right)
$$

where $\lambda_{\theta \mid c_{0}}$ is the hoop stretch at $r=c_{0}$. Also, from Eq. S18, the hydrostatic pressure in $\sigma_{r r}=$ $2 \mu_{\text {skin }} \lambda_{r}^{\alpha} / \alpha-p(r)$ can be solved as

$$
p(r)=\frac{2 \mu_{\text {skin }}}{\alpha\left(\lambda_{z} \lambda_{\theta}\right)^{\alpha}}-\left[\phi\left(\lambda_{z}, \lambda_{\theta}\right)-\phi\left(\lambda_{z}, \lambda_{\theta \mid a_{0}}\right)\right]
$$

Therefore, the Cauchy stress in the z-direction can be expressed as

$$
\sigma_{z}=\frac{2 \mu_{\text {skin }} \lambda_{z}^{\alpha}}{\alpha}-p(r)=\frac{2 \mu_{\text {skin }} \lambda_{z}^{\alpha}}{\alpha}-\frac{2 \mu_{\text {skin }}}{\alpha\left(\lambda_{z} \lambda_{\theta}\right)^{\alpha}}+\left[\phi\left(\lambda_{z}, \lambda_{\theta}\right)-\phi\left(\lambda_{z}, \lambda_{\theta \mid a_{0}}\right)\right]
$$

To solve Eq. S21, instead of assuming $\sigma_{z}=0$, we adopt the relaxed boundary condition of zero resultant force at the arbitrary z-plane (88) as 


$$
0=\int_{a_{1}}^{c_{1}} \sigma_{z} r d r=\int_{\lambda_{\theta \mid a_{0}}}^{\lambda_{\theta \mid c_{0}}} \sigma_{z} \frac{a_{0}^{2} \lambda_{\theta}\left(1-\lambda_{z} \lambda_{\theta \mid a_{0}}^{2}\right)}{\left(1-\lambda_{z} \lambda_{\theta}^{2}\right)^{2}} d \lambda_{\theta}
$$

where the incompressibility enforces that

$$
\lambda_{z}\left(\lambda_{\theta \mid c_{0}}^{2} c_{0}^{2}-\lambda_{\theta \mid a_{0}}^{2} a_{0}^{2}\right)=c_{0}^{2}-a_{0}^{2}
$$

Therefore, one can solve three unknowns $\lambda_{z}, \lambda_{\theta \mid c_{0}}, \lambda_{\theta \mid a_{0}}$ with Eqs. S19, S22, and S23. Then, we can solve the deformation field

$$
\begin{gathered}
\lambda_{\theta}=\sqrt{\frac{R^{2}-a_{0}^{2}+\lambda_{z} a_{1}^{2}}{\lambda_{z} R^{2}}} \\
\lambda_{r}=\frac{1}{\lambda_{z} \lambda_{\theta}}
\end{gathered}
$$

as well as the stress field

$$
\begin{aligned}
\sigma_{\theta} & =\frac{2 \mu_{\text {skin }} \lambda_{\theta}^{\alpha}}{\alpha}-p(r) \\
\sigma_{r} & =\frac{2 \mu_{\text {skin }} \lambda_{r}^{\alpha}}{\alpha}-p(r)
\end{aligned}
$$

which are plotted in fig. S13. It is apparent that when the out boundary is considerably large (i.e., $\left.c_{0} / a_{0} \gg 1\right)$, the converged analytical solutions show that hoop stretch at wound edge $\left.\lambda_{\theta}\right|_{a_{0}}=$ $a_{1} / a_{0}=1.09$. In other words, the size of wound becomes 1.09 times larger than the initial size (i.e., $a_{1} / a=1.05$ ) due to the presence of pre-strain in the native skin.

\subsection{Contraction of wound by the strain-programmable patch}

Next, we can solve $a_{\text {closure }}$ after applying the strain-programmable patch to the wounded skin. Analytical solutions can be obtained when the strain-programmable patch has the same size as that of the enlarged wound (i.e., $\lambda_{\text {patch }}^{\text {pre }} b_{0}=a_{1}=1.05 a$ ) without overlapping area. Let $\sigma_{r \text {, patch }}^{i}$ and $\sigma_{r, \text { skin }}^{i}$ denote the interfacial radial stress on the strain-programmable patch and the skin, respectively. The force balance requires that

$$
\sigma_{r, \text { patch }}^{i} H_{\text {patch }}=\sigma_{r, \text { skin }}^{i} H_{\text {skin }}
$$

where $H_{\text {patch }}$ and $H_{\text {skin }}$ are thickness of the strain-programmable patch and the skin, respectively. As for the skin, stress boundary conditions at $r=\left.a_{\text {closure now changes to } \sigma_{r r}}\right|_{r=a_{\text {closure }}}=\sigma_{r \text {,skin }}^{i}$. Similar analysis can be performed to find three equations as 


$$
\begin{gathered}
\sigma_{\infty}-\sigma_{r, \text { skin }}^{i}=\phi\left(\lambda_{z}, \lambda_{\theta}\right)-\phi\left(\lambda_{z}, \lambda_{\theta \mid a_{0}}\right) \\
0=\int_{a_{\text {closure }}}^{c_{2}} \sigma_{z} r d r \\
\lambda_{z}\left(\lambda_{\theta \mid c_{0}}^{2} c_{0}^{2}-\lambda_{\theta \mid a_{0}}^{2} a_{0}^{2}\right)=c_{0}^{2}-a_{0}^{2}
\end{gathered}
$$

from which one can solve $\lambda_{z}, \lambda_{\theta \mid a_{0}}=a_{\text {closure }} / a$, and $\lambda_{\theta \mid c_{0}}=c_{2} / c_{0}$ as a function of $\lambda_{\text {patch. }}^{\text {pre }}$ The analytical solution from Eq. S27 are plotted in Figures 4D and S15D which validates the finiteelement results. Note that the analytical solutions are only available when the strain-programmable patch size equals to $a_{1}=1.1 a$ for diabetic mouse skin and $a_{1}=1.05 a$ for human skin. For the strain-programmable patch with larger sizes, finite-element modeling-based analysis is required as discussed in the following section.

\section{Finite-element modeling}

To quantitatively analyze the closure and stress remolding of wounds by the strain-programmable patch larger than the wound size (i.e., $b_{0}>1.1 a$ for diabetic mouse skin, $b_{0}>1.05 a$ for human skin), we develop 2D axisymmetric finite-element models based on a commercially-available software (ABAQUS/Standard 2017, Dassault Systèmes ${ }^{\circledR}$ ) for both diabetic mouse skin and porcine skin. The finite-element setups are illustrated in fig. S14. The diabetic mouse skin was modeled as an incompressible Ogden hyperelastic solid (fitting parameters: $\mu_{\text {mouse }}=87.5 \mathrm{kPa}, \alpha_{\text {mouse }}=7$ ) with $3.5 \%$ tensile pre-strain. The human skin was modeled as an incompressible Ogden hyperelastic solid (fitting parameters: $\mu_{\text {human }}=40 \mathrm{kPa}, \alpha_{\text {human }}=20$ ) with $3.5 \%$ tensile prestrain (36). The strain-programmable patch was modeled as a neo-Hookean solid (fitting parameter: $\mu_{\text {patch }}^{\infty}=17.2 \mathrm{kPa}$ ) with varying patch sizes and pre-stretches for strain-programming. Three mechanical quantities, (i) wound closure ratio defined as $a_{\text {closure }} / a$ (Fig. 4D and fig. S15D), (ii) normalized hoop stress $\sigma_{\theta} / \sigma_{\infty}$ (Fig. $4 \mathrm{E}$ and fig. S15E), and (iii) normalized radial stress $\sigma_{r} / \sigma_{\infty}$ (Fig. 4F and fig. S15F) are obtained from the finite-element models for both diabetic mouse skin and human skin. 


\section{Supplementary Figures}

A

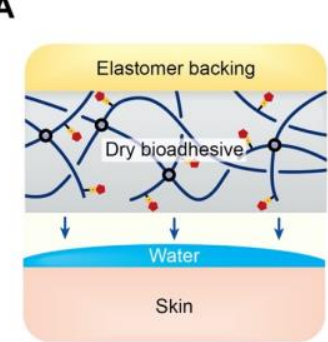

(1) Wet skin tissue

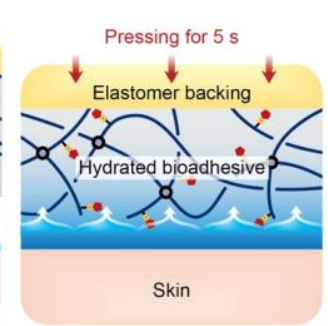

2 Dry-crosslinking process

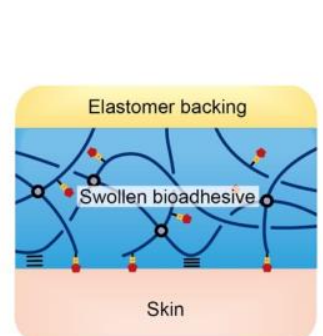

(3) Robust adhesion formation
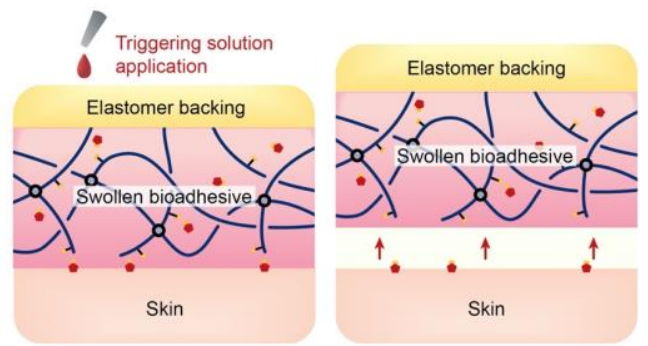

$>$ Rapid \& robust wet adhesion

(4) Triggered de-crosslinking

(5) On-demand detachment - 2 polymer network

- Covalent crosslinking functional groups

Triggerable detachment

\section{B}
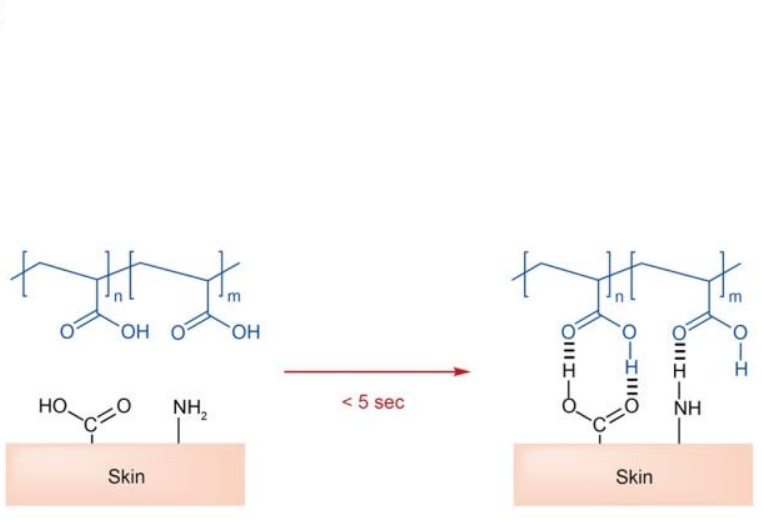

C

Physical crosslinking

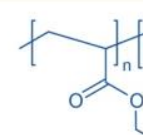

Cleavable

_. Cleavable

physical crosslinks

covalent crosslinks
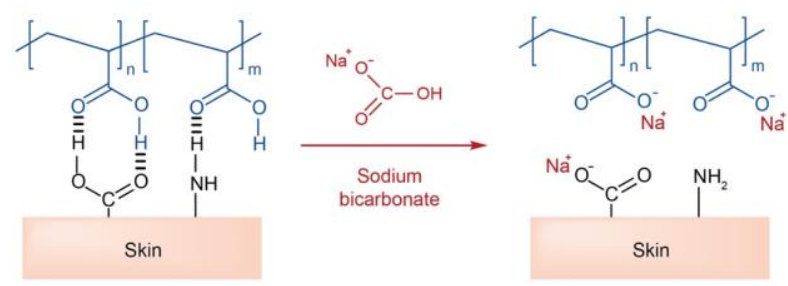

Triggerable cleavage of physical crosslinks

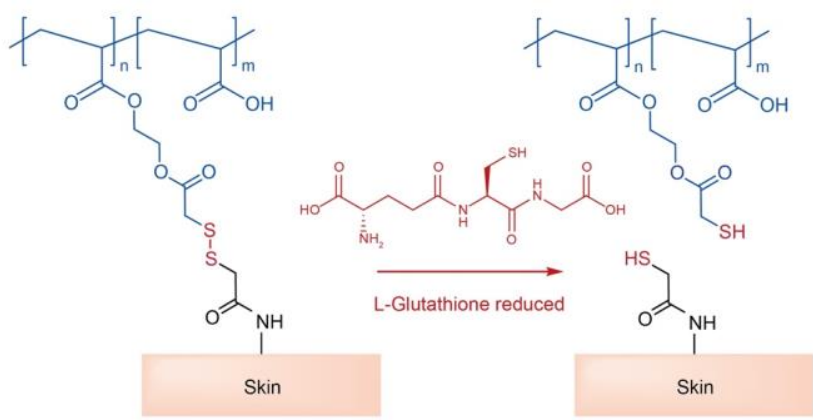

Triggerable cleavage of covalent crosslinks

Fig. S1. Rapid wet adhesion and triggerable detachment of a strain-programmable patch. (A) Schematics illustration for rapid wet adhesion and triggerable detachment of the strainprogrammable patch. (B) Chemistry of physical and covalent crosslinks for rapid wet adhesion. (C) Chemistry of on-demand triggerable detachment. 

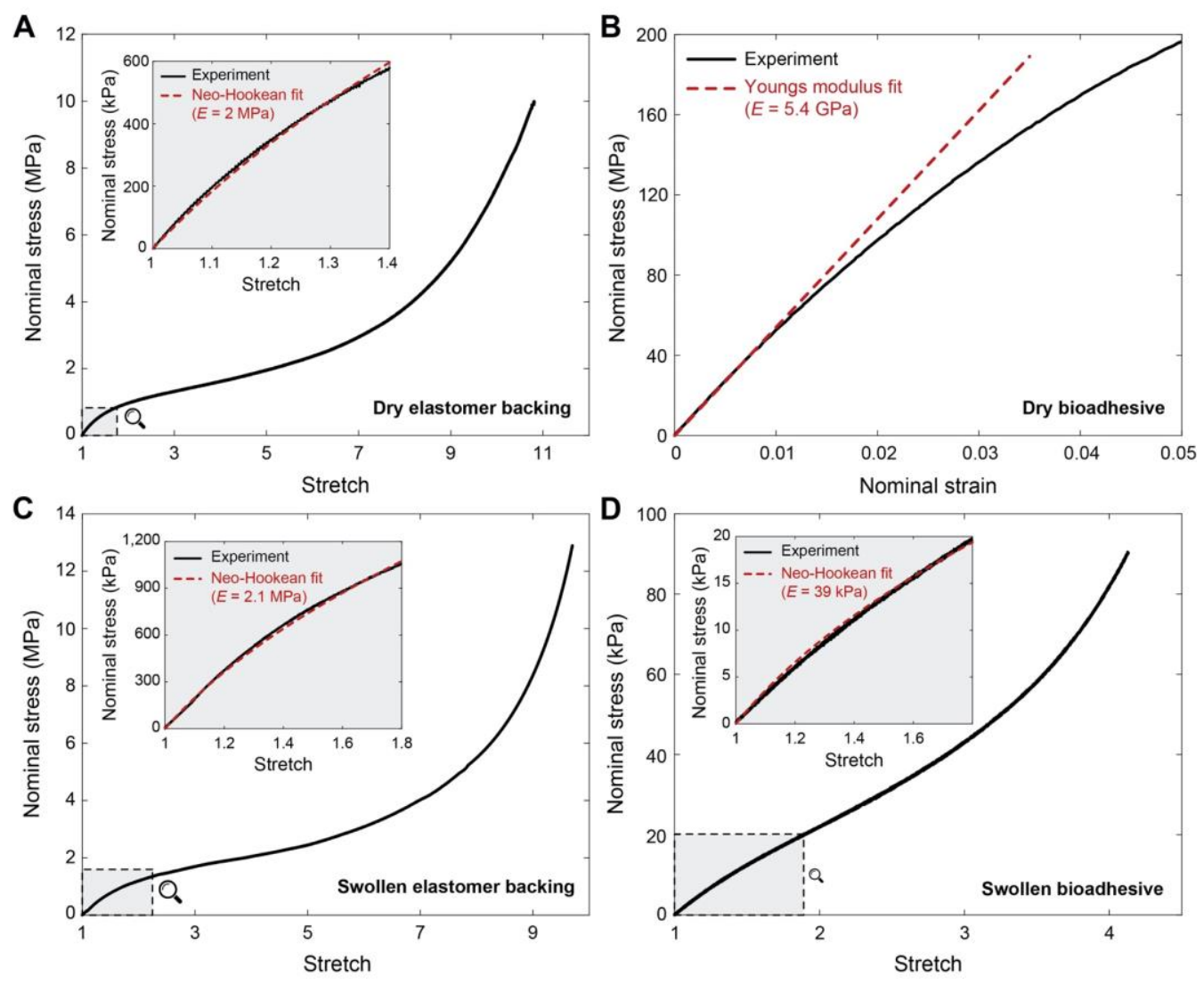

Fig. S2. Mechanical properties of elastomer backing and bioadhesive. (A and B) Nominal stress vs. stretch curves for dry elastomer backing (A) and bioadhesive (B). (C and D) Nominal stress vs. stretch curves for swollen elastomer backing (C) and bioadhesive (D). 


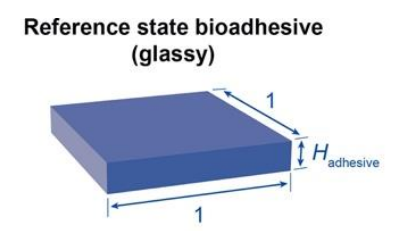

As-prepared bioadhesive (rubbery)

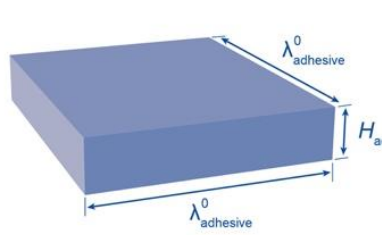

Pre-stretched assembly
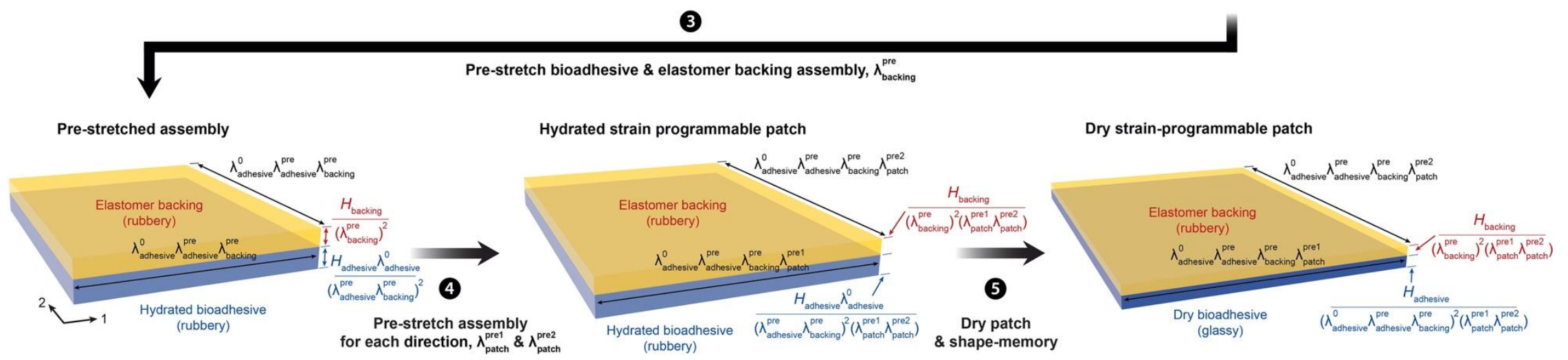

Pre-stretched bioadhesive with elastomer backing resin

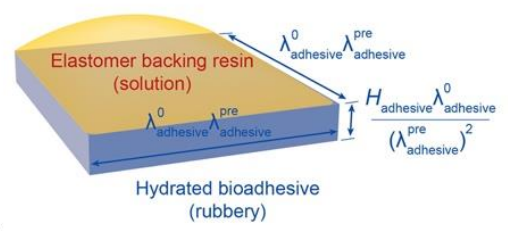

3

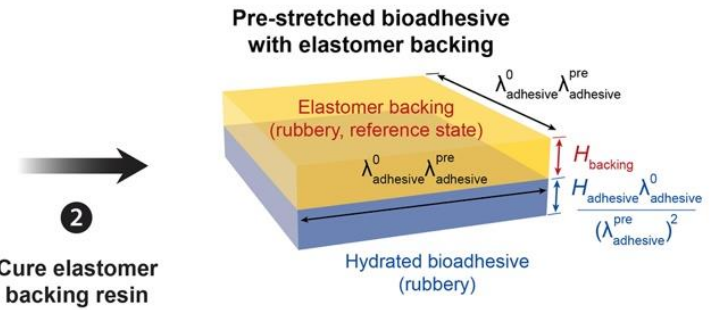

backing resin

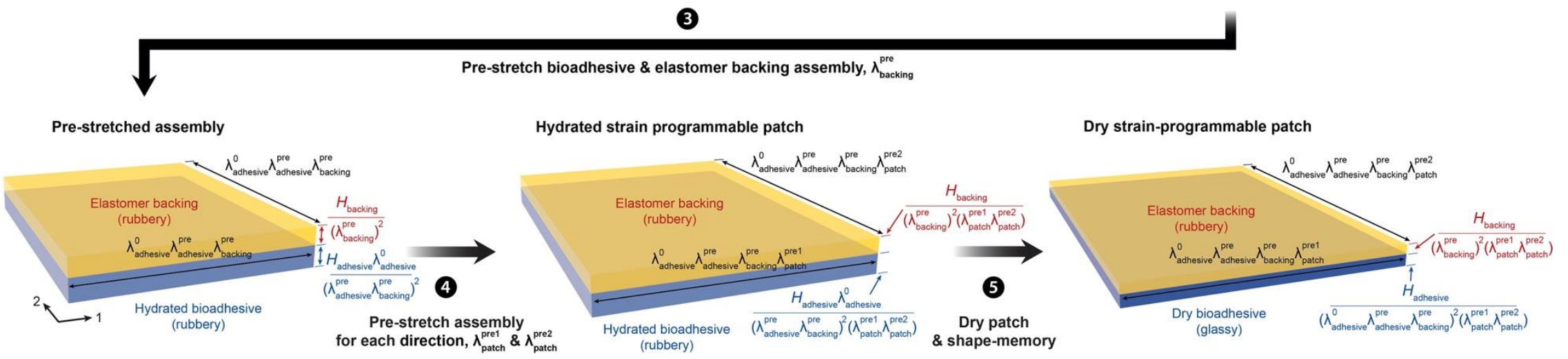

Fig. S3. Fabrication of the strain-programmable patch. (1) Spin-coat elastomer backing resin on an as-prepared bioadhesive and pre-stretch the as-prepared bioadhesive by ratio of $\lambda_{\text {adhes }}^{\text {pre }}$ (2) Cure elastomer backing resin while keeping the bioadhesive hydrated. (3) Pre-stretch the bioadhesive and elastomer backing assembly by ratio of $\lambda_{\text {backing }}^{\text {pre }}$ (4) Pre-stretch the bioadhesive patch along two inplane directions by ratios of $\lambda_{\text {patch }}^{\text {pre1 }}$ and $\lambda_{\text {patch }}^{\text {pre2 }}$, respectively. (5) Dry the bioadhesive for shape-memory to finalize fabrication of the strain-programmed patch. 

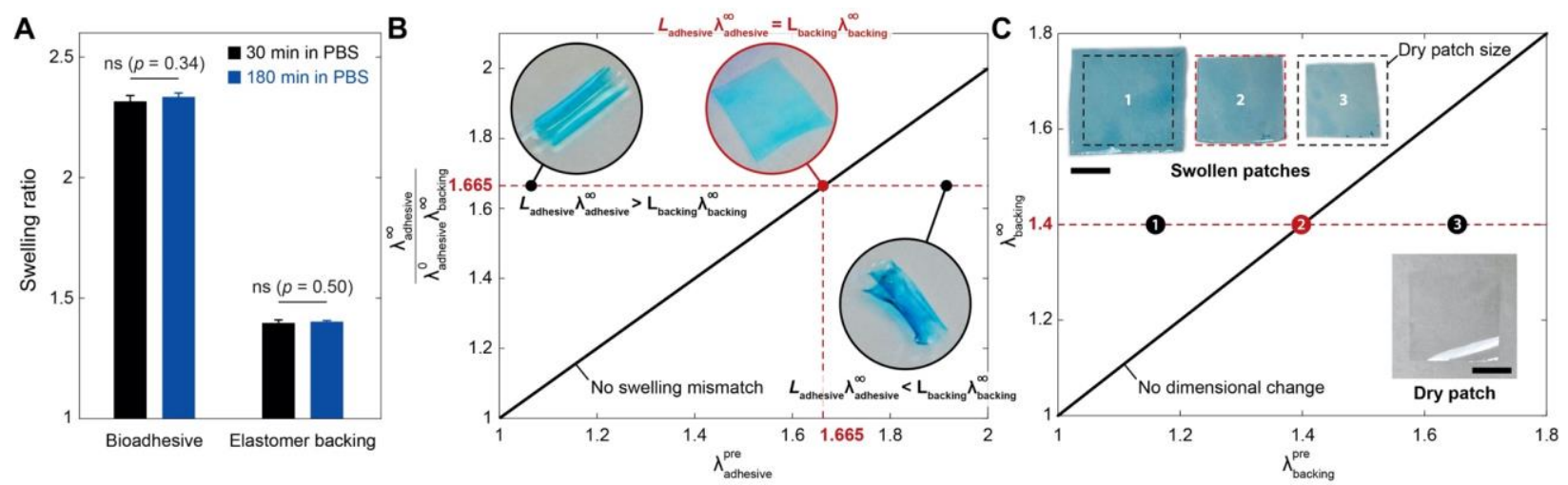

Fig. S4. Swelling of strain-programmable patch. (A) Swelling ratios of elastomer backing and bioadhesive in wet physiological environment. (B) Swelling mismatch canceling between elastomer backing and bioadhesive in the strain-programmable patch. (C) Swelling canceling of the strain-programmable patch. Values in A represent the means \pm SD $(n=4) . P$ values are determined by a Student's $t$ test; ns, not significant. Scale bars, $10 \mathrm{~mm}(\mathrm{C})$. 

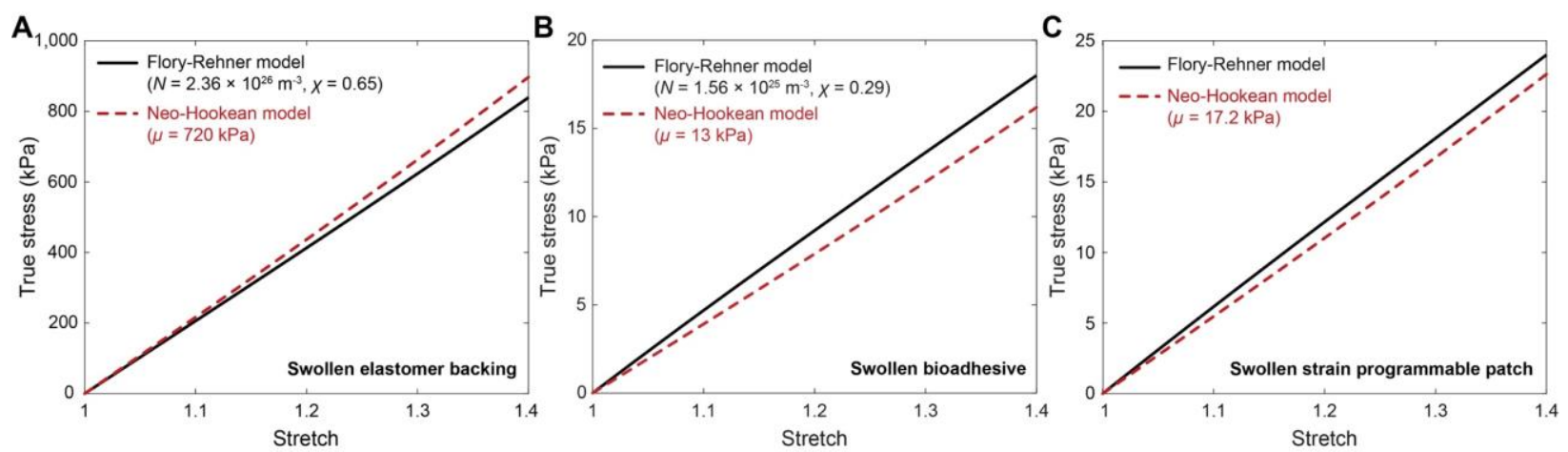

Fig. S5. Comparison between Flory-Rehner and neo-Hookean models. (A to C) True stress vs. stretch obtained based on Flory-Rehner and neo-Hookean models for swollen elastomer backing (A), bioadhesive (B), and strain-programmable patch (C). 
A

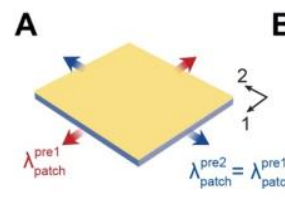

Isotropically strain programmed patch

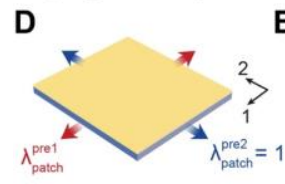

Anisotropically strain programmed patch

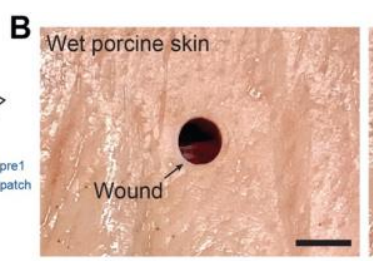

E

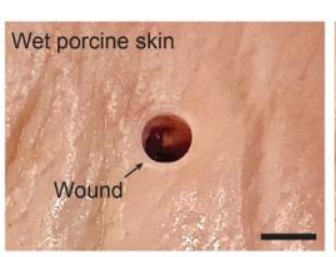

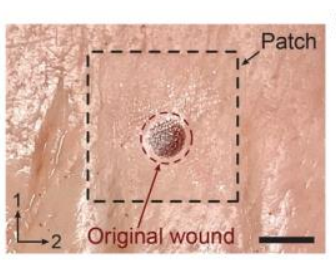

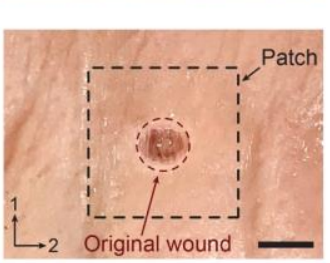

C Wet diabetic mouse skin

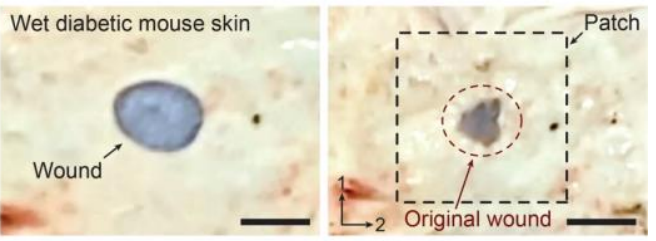

$\mathbf{F}$

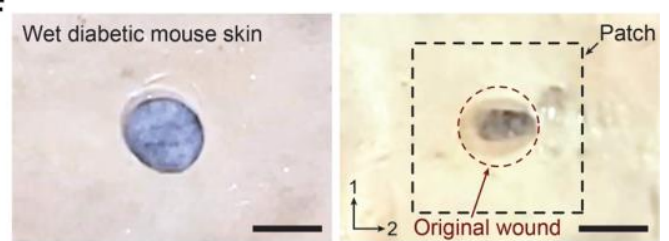

Fig. S6. Isotropic and anisotropically strain-programming of the patch. (A) Schematic illustration for the isotropically strain-programmed patch. (B and C) Isotropically strainprogrammed patches on a porcine skin (B) and a diabetic mouse skin (C) with circular wound. (D) Schematic illustration for the anisotropically strain-programmed patch. (E and $\mathbf{F})$ Anisotropically strain-programmed patches on a porcine skin $(\mathrm{E})$ and a diabetic mouse skin $(\mathrm{F})$ with circular wound. Scale bars, $10 \mathrm{~mm}$ (B and $\mathrm{E})$; and $5 \mathrm{~mm}(\mathrm{C}$ and $\mathrm{F})$. 

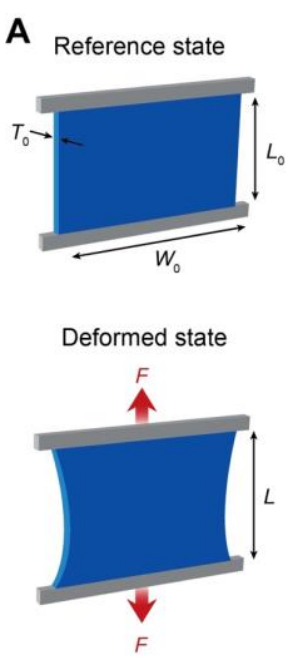

Unnotched sample
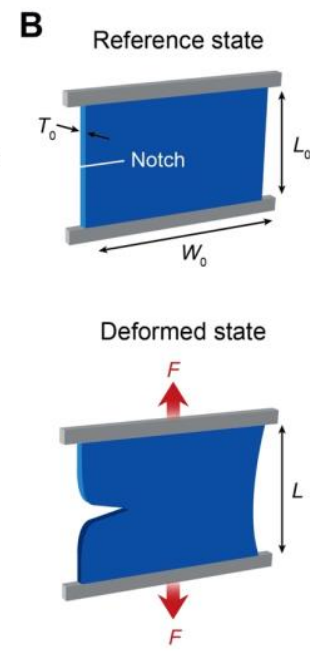

Notched sample
C

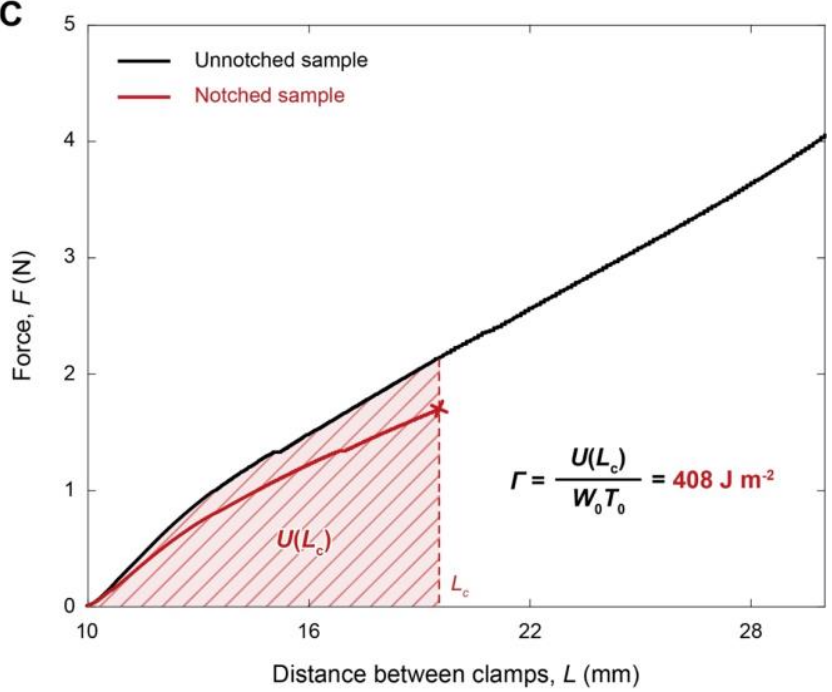

Fig. S7. Fracture toughness of strain-programmable patch. (A and B) Schematic illustrations of pure-shear test for an unnotched sample (A) and a notched sample (B). (C) Force vs. distance between clamps for the unnotched and notched swollen strain-programmable patch for fracture toughness measurement. $L_{\mathrm{c}}$ indicates the critical distance between the clamps at which the notch turns into a running crack. The measured fracture toughness of the strain-programmable patch is $408 \mathrm{~J} \mathrm{~m}^{-2}$. 

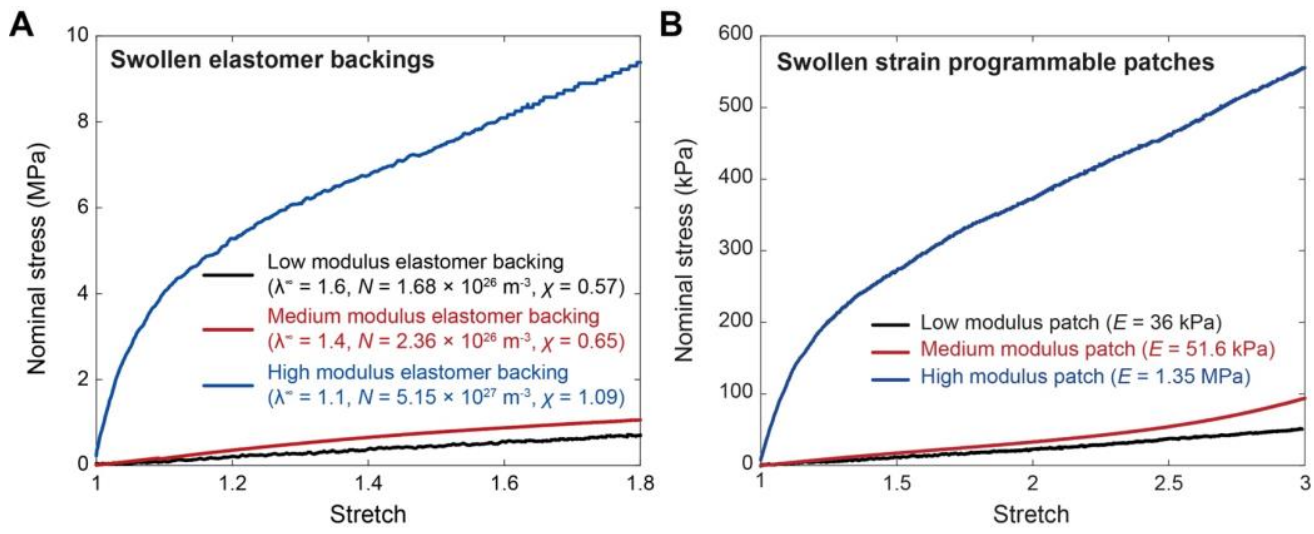

Fig. S8. Various elastomer backings for the strain-programmable patch. (A) Nominal stress vs. stretch curves for various elastomer backing materials. (B) Nominal stress vs. stretch curves for the strain-programmable patch with various elastomer backing materials. 
bioRxiv preprint doi: https://doi.org/10.1101/2021.06.07.447423; this version posted June 7, 2021. The copyright holder for this preprint (which was not certified by peer review) is the author/funder, who has granted bioRxiv a license to display the preprint in perpetuity. It is made available under aCC-BY-NC-ND 4.0 International license.

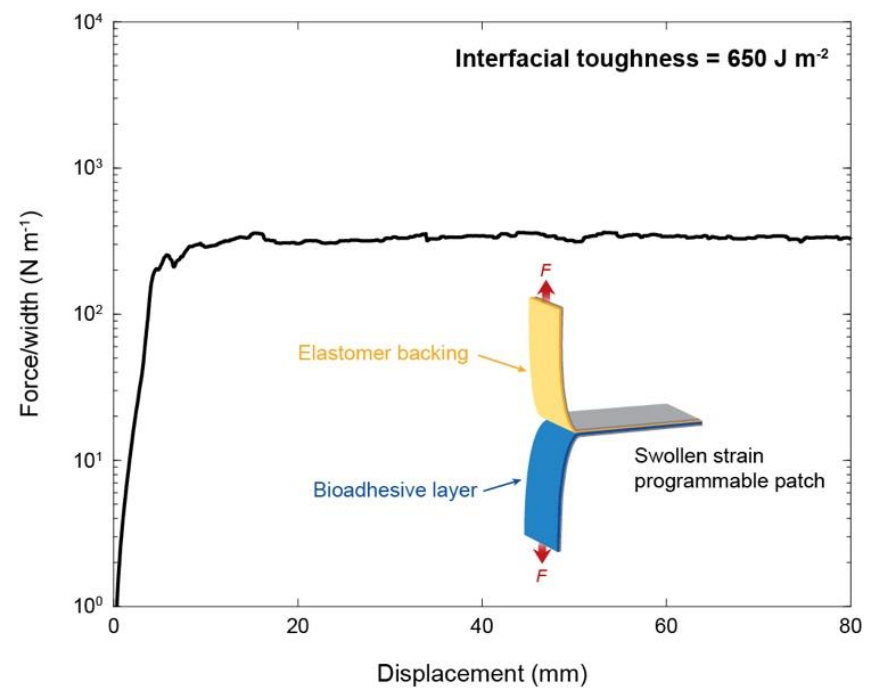

Fig. S9. Interfacial toughness between elastomer backing and bioadhesive in the strainprogrammable patch. The measured interfacial toughness between swollen elastomer backing and bioadhesive is $650 \mathrm{~J} \mathrm{~m}^{-2}$. 


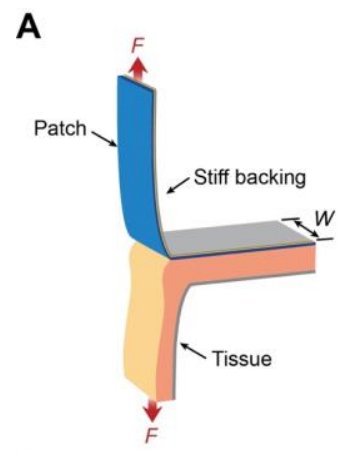

C
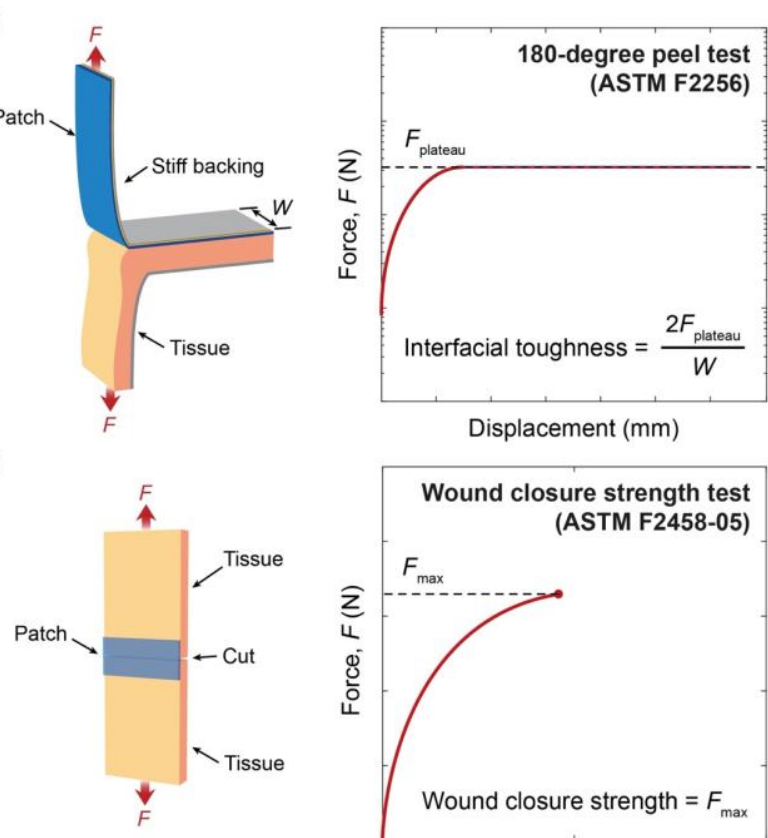

B

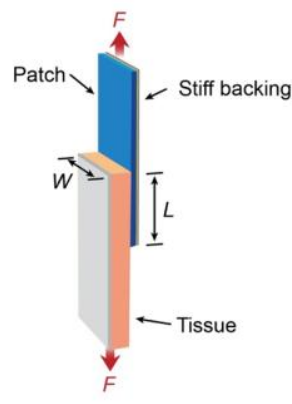

D

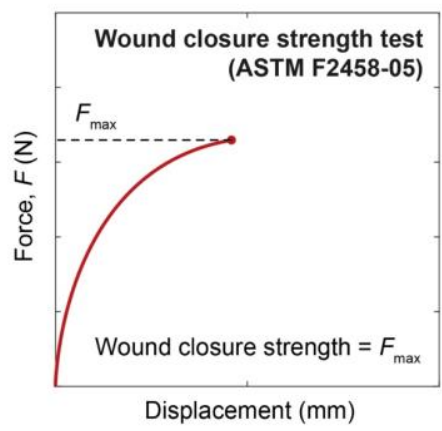

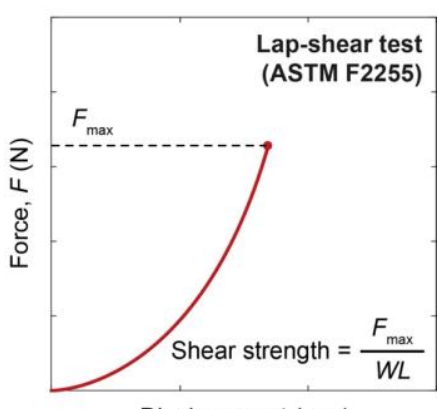

Displacement (mm)

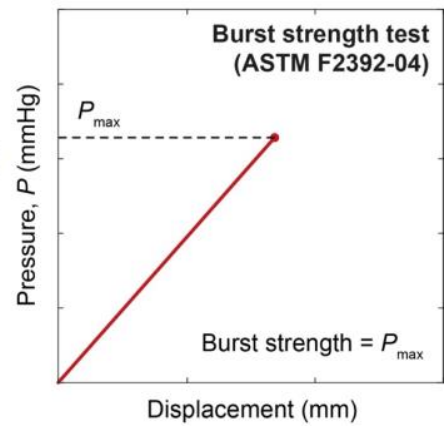

Fig. S10. Mechanical testing setups for evaluation of adhesion performance. (A) Testing setup for interfacial toughness measurements based on the standard 180-degree peel test (ASTM F2256). (B) Testing setup for shear strength measurements based on the standard lap-shear test (ASTM F2255). (C) Testing setup for wound closure strength measurements based on the standard tensile test (ASTM F2458-05). (D) Testing setup for burst strength measurements based on the standard burst pressure test (ASTM F2392-04). 
bioRxiv preprint doi: https://doi.org/10.1101/2021.06.07.447423; this version posted June 7, 2021. The copyright holder for this preprint (which was not certified by peer review) is the author/funder, who has granted bioRxiv a license to display the preprint in perpetuity. It is made available under aCC-BY-NC-ND 4.0 International license.
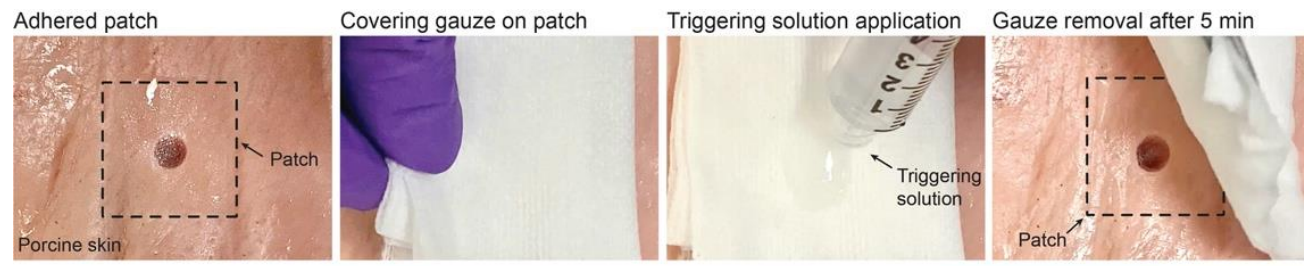

Patch removal from skin

Triggerable on-demand detachment of strain-programmable patch

Fig. S11. Triggerable on-demand detachment of the strain-programmable patch. 
A Reference state

Skin

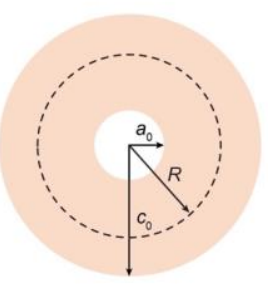

B

Patch
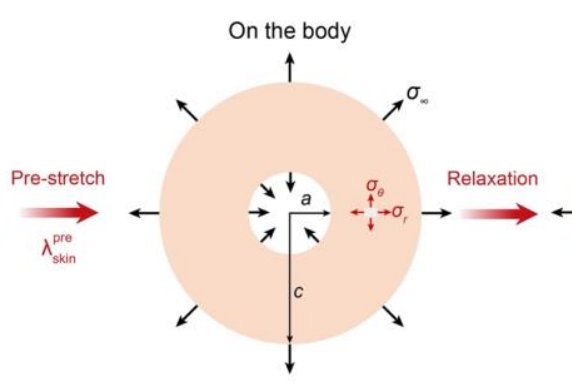

Pre-stretch

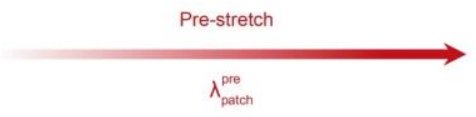

After wounding

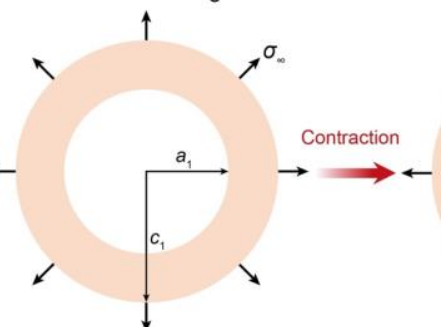

Contraction

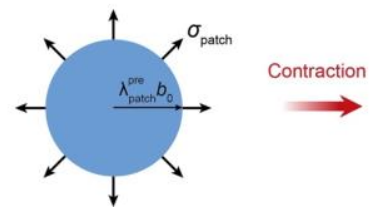

Patch application

$\uparrow$
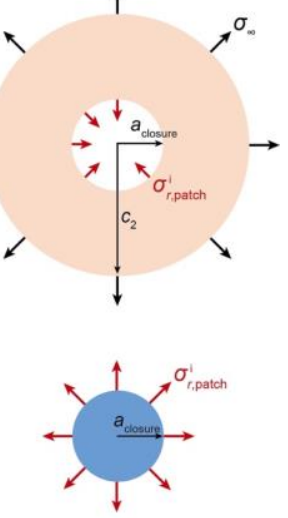

Fig. S12. Schematic configuration for the analytical modeling. ( $A$ and $B$ ) Axisymmetric configuration of the wounded skin (A) and the strain-programmable patch (B). 


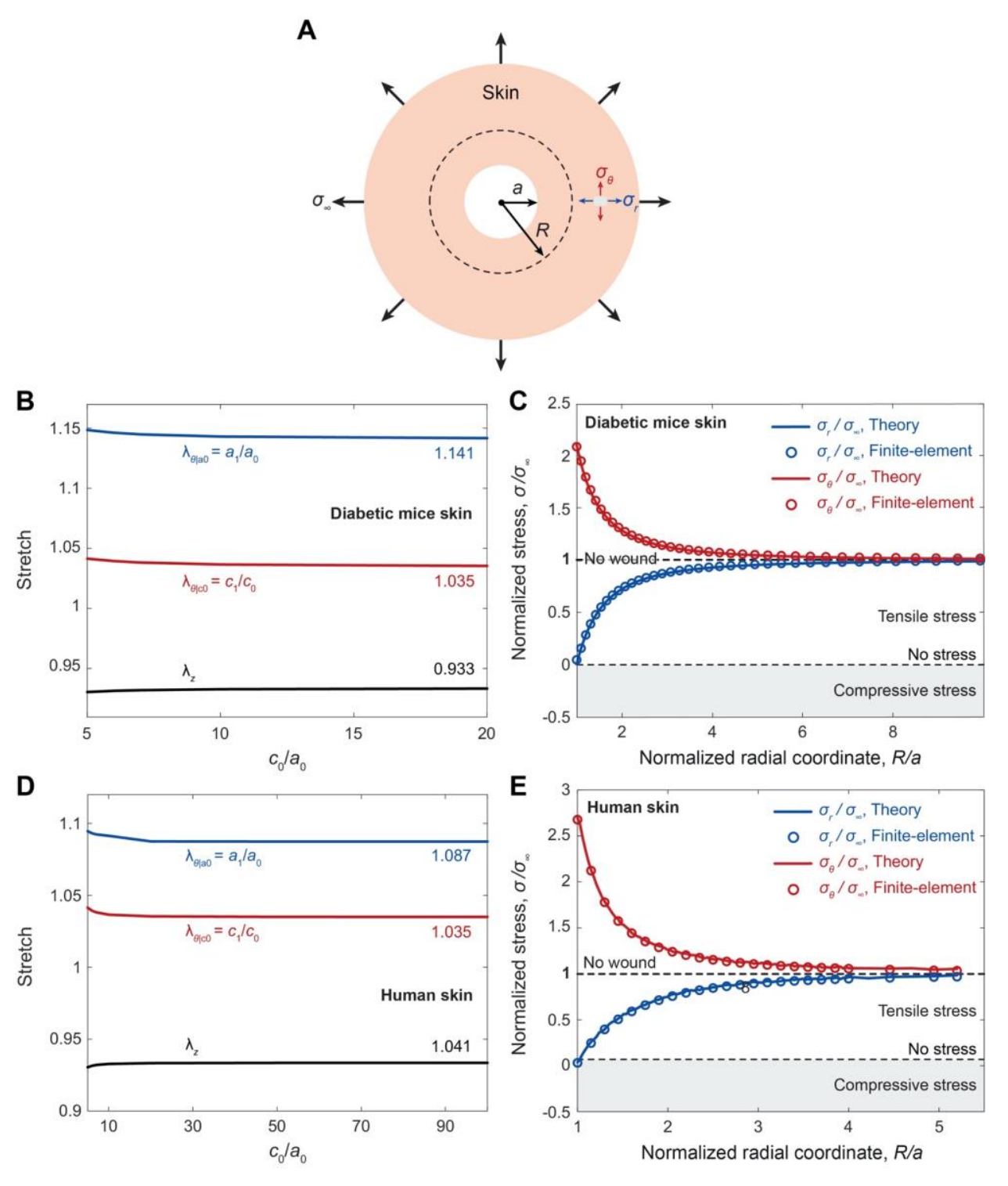

Fig. S13. Comparison of analytical and finite-element analyses. (A) Schematic illustration for axisymmetric configuration for analytical and finite-element analyses. (B) Analytically solved stretches of the wounded diabetic mouse skin as a function of $c_{0} / a_{0}$. (C) Stress distribution within the wounded diabetic mouse skin calculated from the analytical solutions (solid lines) and the corresponding finite-element results (circles). (D) Analytically solved stretches of the wounded human skin as a function of $c_{0} / a_{0}$. (E) Stress distribution within the wounded human skin calculated from the analytical solutions (solid lines) and the corresponding finite-element results (circles). 
bioRxiv preprint doi: https://doi.org/10.1101/2021.06.07.447423; this version posted June 7, 2021. The copyright holder for this preprint (which was not certified by peer review) is the author/funder, who has granted bioRxiv a license to display the preprint in perpetuity. It is made available under aCC-BY-NC-ND 4.0 International license.

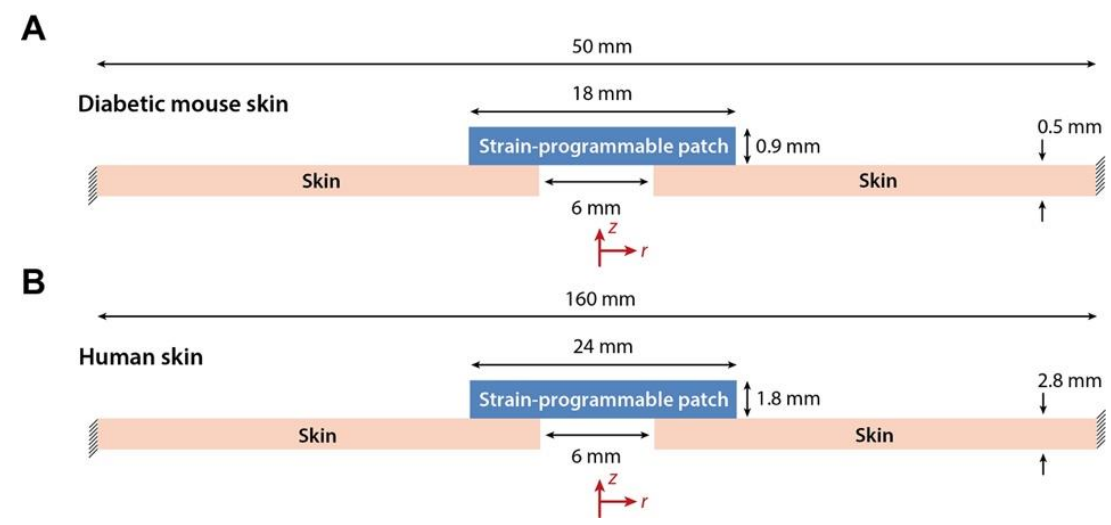

Fig. S14. Finite-element modeling for wound closure by the strain-programmable patch. (A and B) Finite-element setups for diabetic mouse skin (A) and human skin (B) with wound and the strain-programmed patch. 

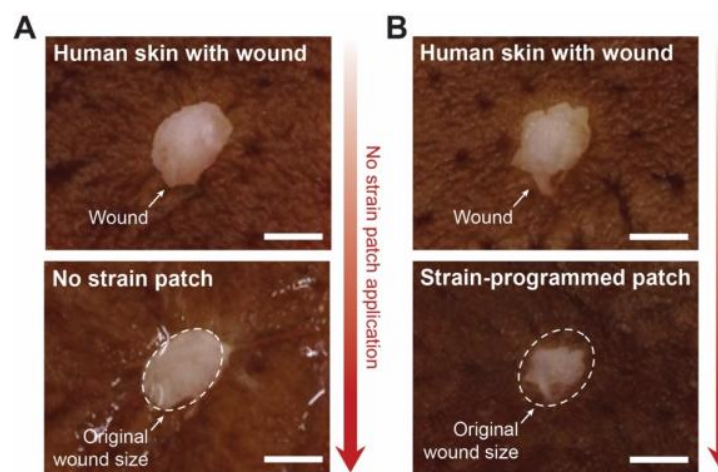

Strain-programmed patch
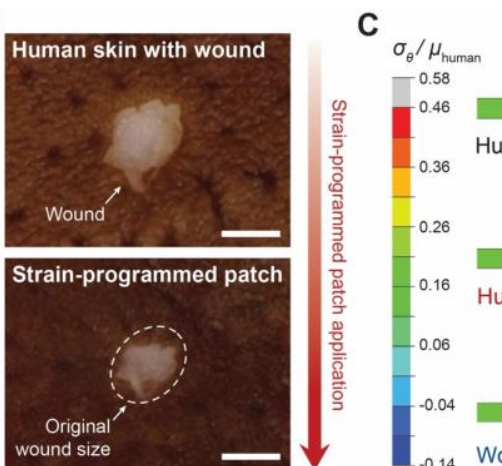

Human skin with pre-strain

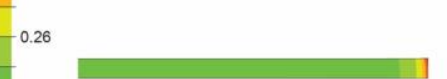

0.16 Human skin with wound

$0.06 \quad$ Strain-programmable patch, $\lambda_{\text {perem }}^{\text {pre }}=1.3$ Strain-programmable patch, $\lambda_{\text {patch }}^{\text {pro }}=1.3$ 14 Wound contraciton by the strain-programmable patch
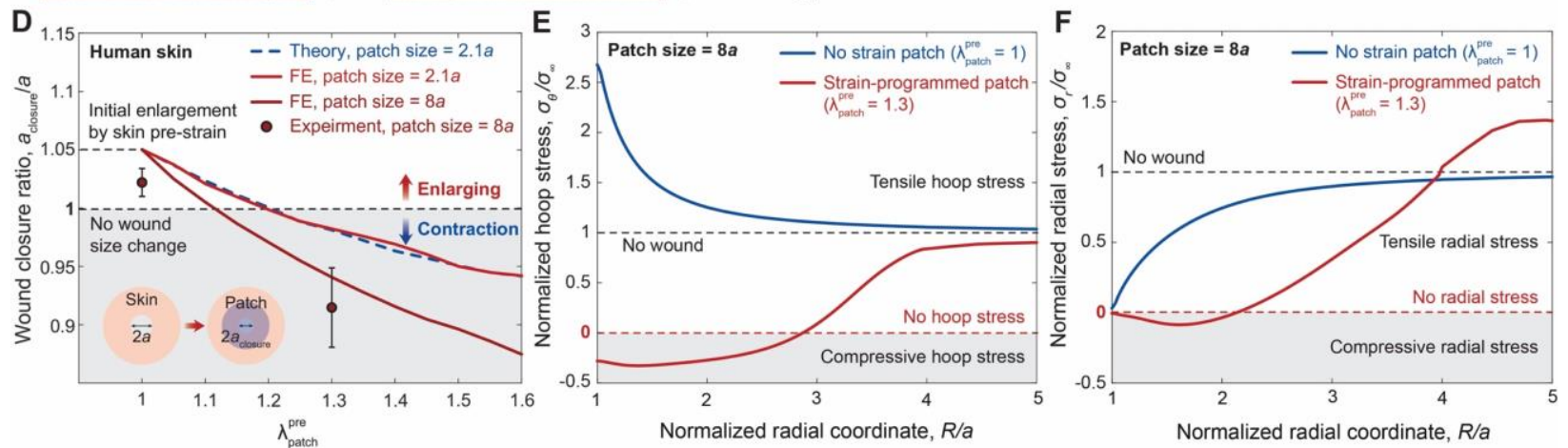

Fig. S15. Programmable closure and stress remodeling of ex vivo human skin wounds. (A and B) Images of a no strain patch with $\lambda_{\text {patch }}^{\text {pre }}=1$ (A) and a strain-programmed patch with $\lambda_{\text {patch }}^{\text {pre }}=1.3$ (B) on ex vivo human skin with a circular wound. (C) Representative finite-element results of the human skin with a strain-programmable patch with $\lambda_{\text {patch }}^{\text {pre }}=1.3$ and patch diameter 4 times of the wound diameter. The shear modulus of the human skin is denoted as $\mu_{\text {human, the hoop }}$ stress in the human skin as $\sigma_{\theta}$, and the residual stress in the intact human skin as $\sigma_{\infty}$. (D) Finiteelement and experimental results for the wound closure ratio as a function of $\lambda_{\text {patch }}^{\text {pre }}$. FE, finiteelement. ( $\mathbf{E}$ and $\mathbf{F}$ ) Finite-element results for the hoop $\sigma_{\theta}(\mathrm{E})$ and the radial $\sigma_{\mathrm{r}}(\mathrm{F})$ stresses around the wound with the strain-programmed patches with varying $\lambda_{\text {patch }}^{\text {pre }}$ Values in $\mathrm{D}$ represent the means $\pm \mathrm{SD}(n=3)$. Scale bars, $5 \mathrm{~mm}(\mathrm{~A}$ and $\mathrm{B})$. 

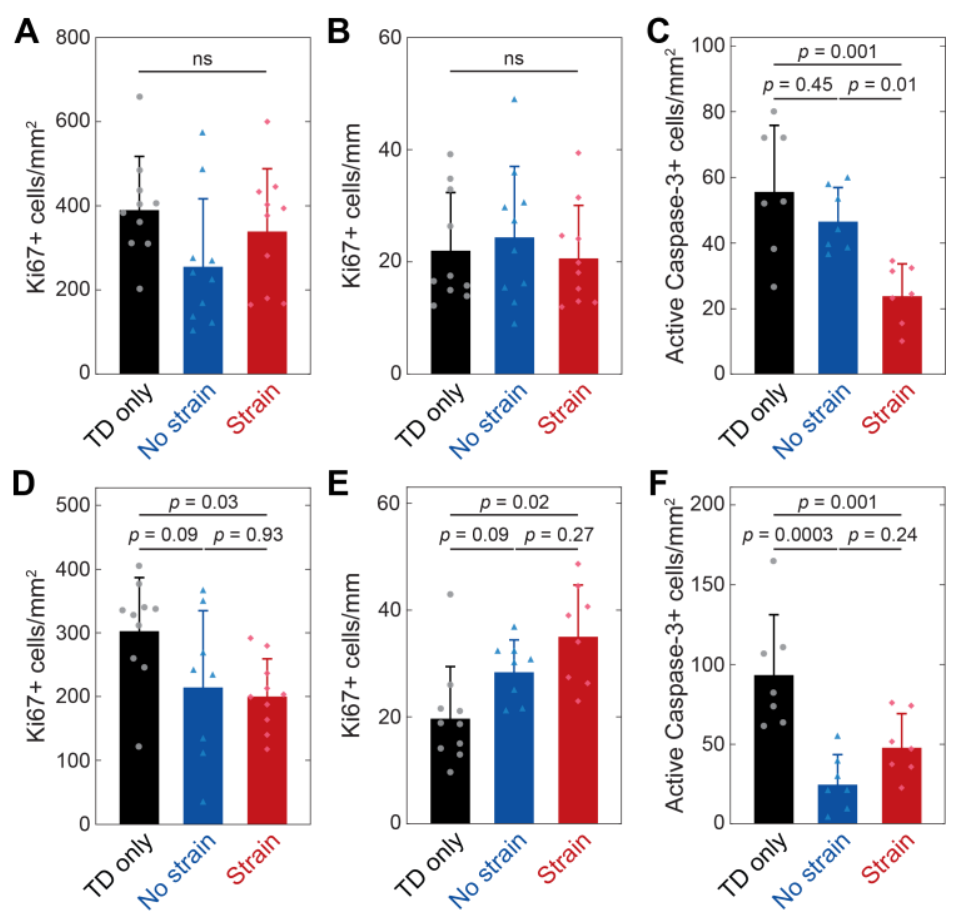

Fig. S16. Wound cells proliferation and apoptosis. (A and B) Quantification of proliferation marker Ki67+ cells in the dermis (A) and the epidermis (B) of day 5 (D5) wounds. (C) Quantification of apoptosis marker active Caspase-3+ cells in the dermis of D5 wounds. (D and E) Quantification of proliferation marker Ki67+ cells in the dermis (D) and the epidermis (E) of day 10 (D10) wounds. (F) Quantification of apoptosis marker active Caspase-3+ cells in the dermis of D10 wounds. Values represent the means $\pm \mathrm{SD}(n=7-10) . P$ values were derived from one way ANOVA with Tukey's post-hoc tests. ns, not significant. 

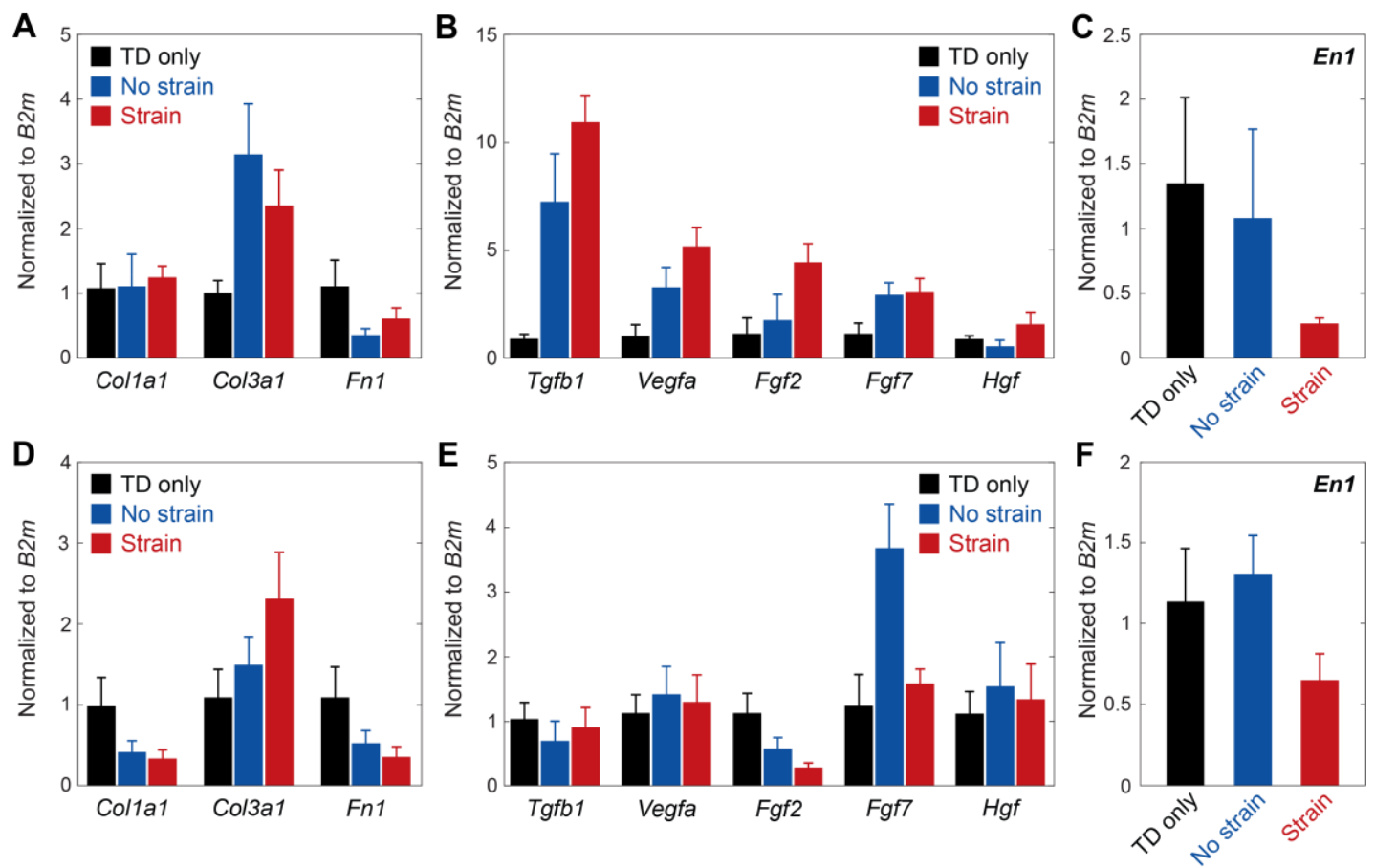

Fig. S17. RT-qPCR gene expression analysis. (A and B) RT-qPCR analysis on day 5 (D5) wounds for ECM-related (A) and growth factor (B) genes. (C) Engrailed-1 (Enl) wound expression on D5. (D and E) RT-qPCR analysis on day 10 (D10) wounds for ECM-related (D) and growth factor (E) genes. (F) Engrailed-1 (Enl) wound expression on D10. Values represent the means $\pm \operatorname{SEM}(n=4-6)$. 
A

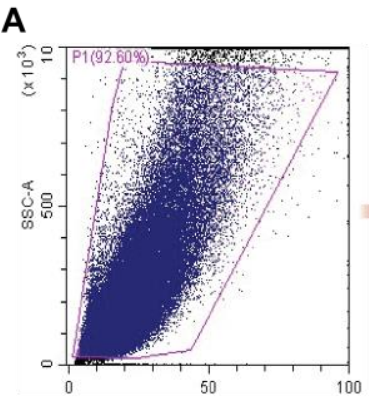

B

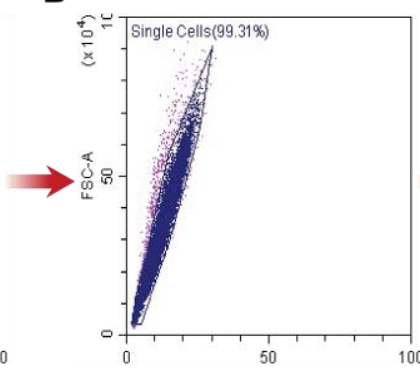

C

F $\begin{gathered}\text { Macrophages } \\ \mathrm{CD} 45+\mathrm{CD} 11 \mathrm{~b}+\mathrm{CD} 64+\mathrm{F} 4 / 80+ \\ \text { Sample } 12: \mathrm{CD64+}\end{gathered}$

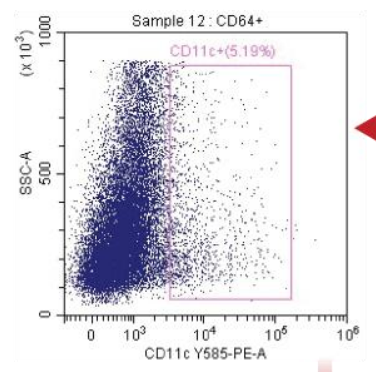

Monocytes-derived DCs H CD45+ MHCllhi CD11b+ CD11C+CD64+
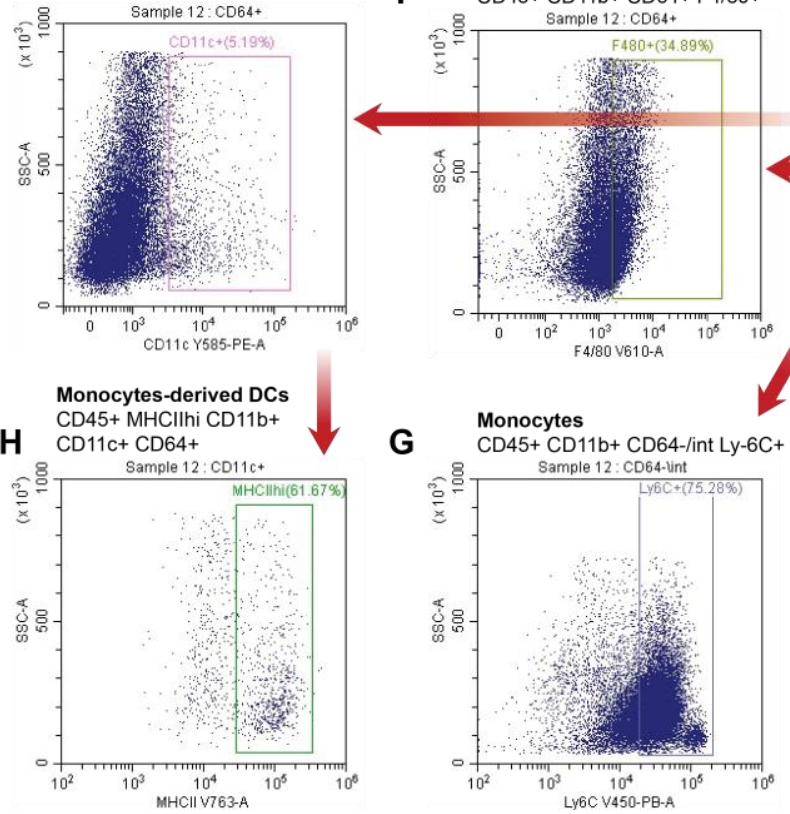

G

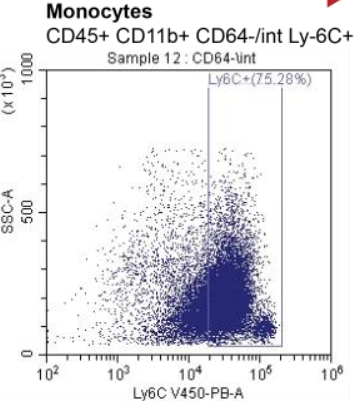

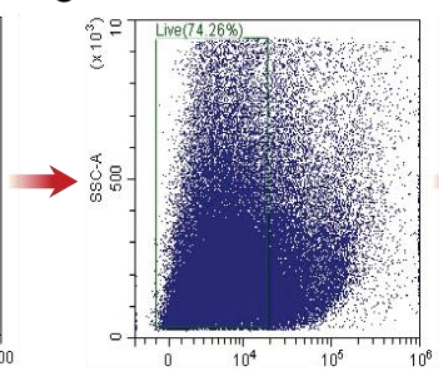

D Immune cells

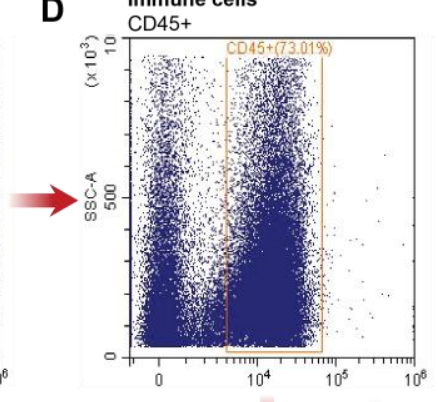$$
\text { . }
$$
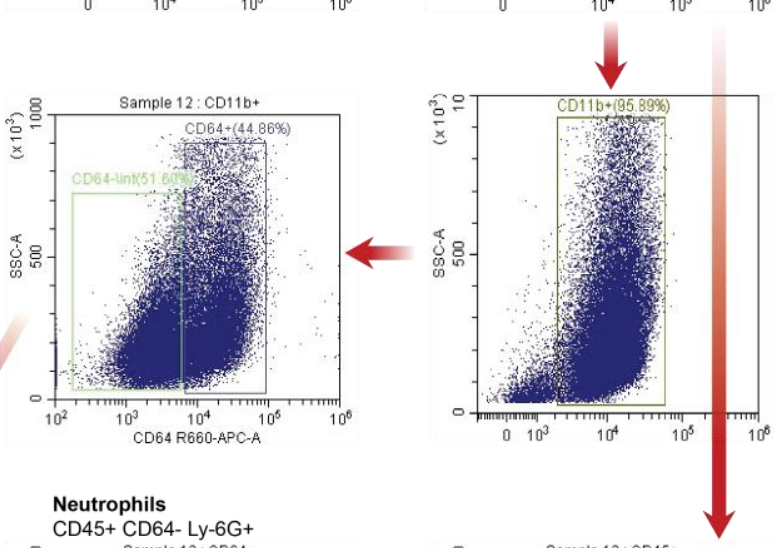

E

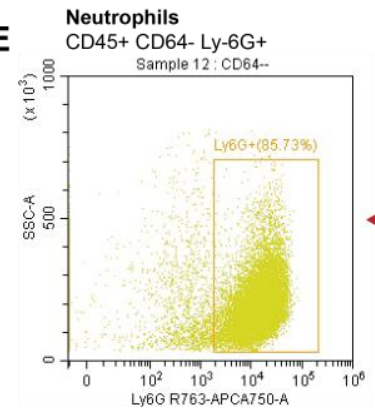

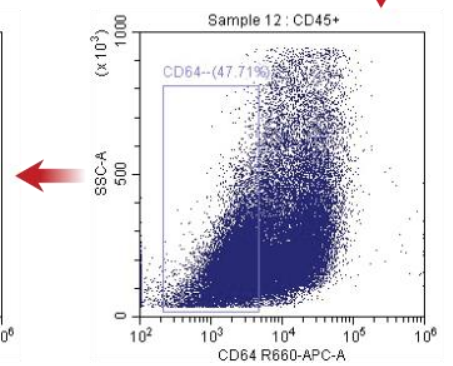

Fig. S18. Gating strategy for the flow cytometry analysis of dissociated wound tissues. (A-H) Forward and side scatter density plot (A) was used for debris exclusion followed by forward scatter area vs forward scatter height density plot (B) for doublet exclusion. Live (C) immune cells (D) were then characterized as neutrophils $(\mathrm{E})$, macrophages $(\mathrm{F})$, monocytes $(\mathrm{G})$, and monocyte derived dendritic cells $(\mathrm{H})$ with appropriate cell surface antibody staining and sequential gating. 
A T-cells

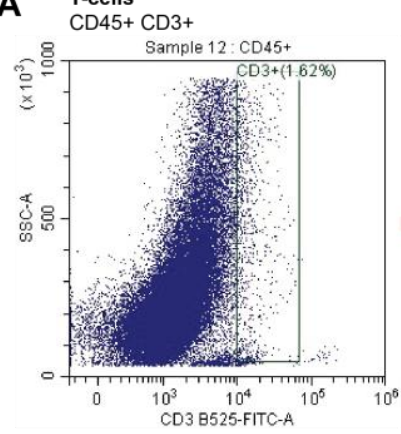
C Macrophage polarization

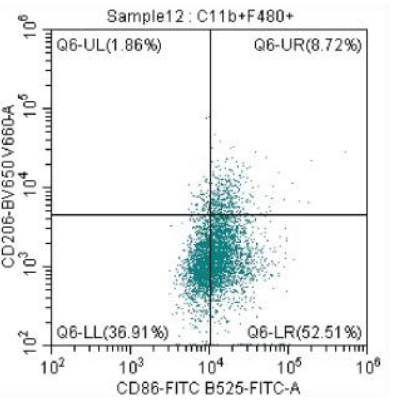

B T-cells subsets $\mathrm{CD} 4$ \& CD8

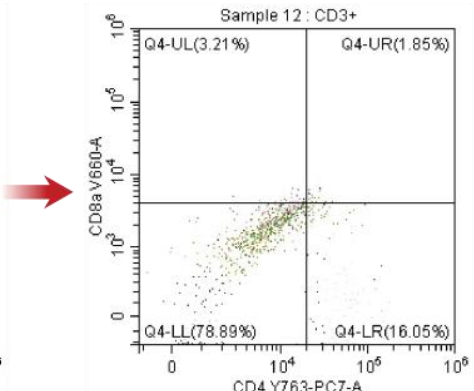

D Macrophage polarization CD163 \& CD80

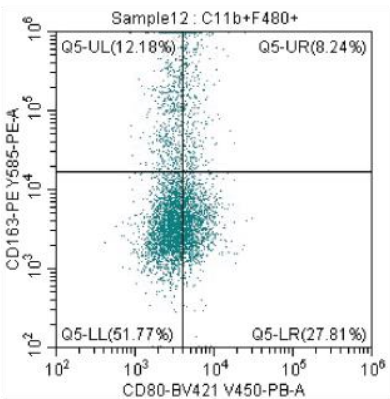

E Macrophage polarization CD301b \& CD80

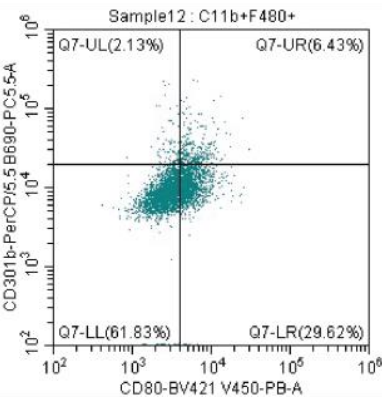

Fig. S19. Additional gating strategy for T-cell subsets and polarized macrophages. (A-E) Single, live, immune cells were characterized as T-cells (A) and further gated according to CD4 and CD8 expression (B). Density plot graphs were also used to distinguish macrophage (single, live, CD45+CD11b+CD64+F4/80+ cells') polarized states (C-E). 
A

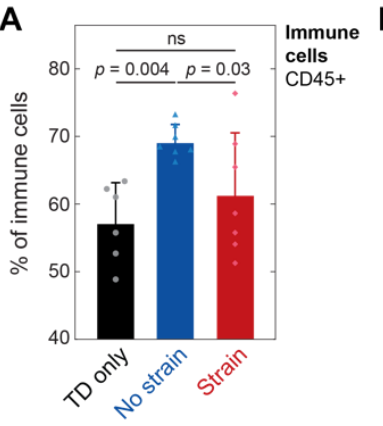

E
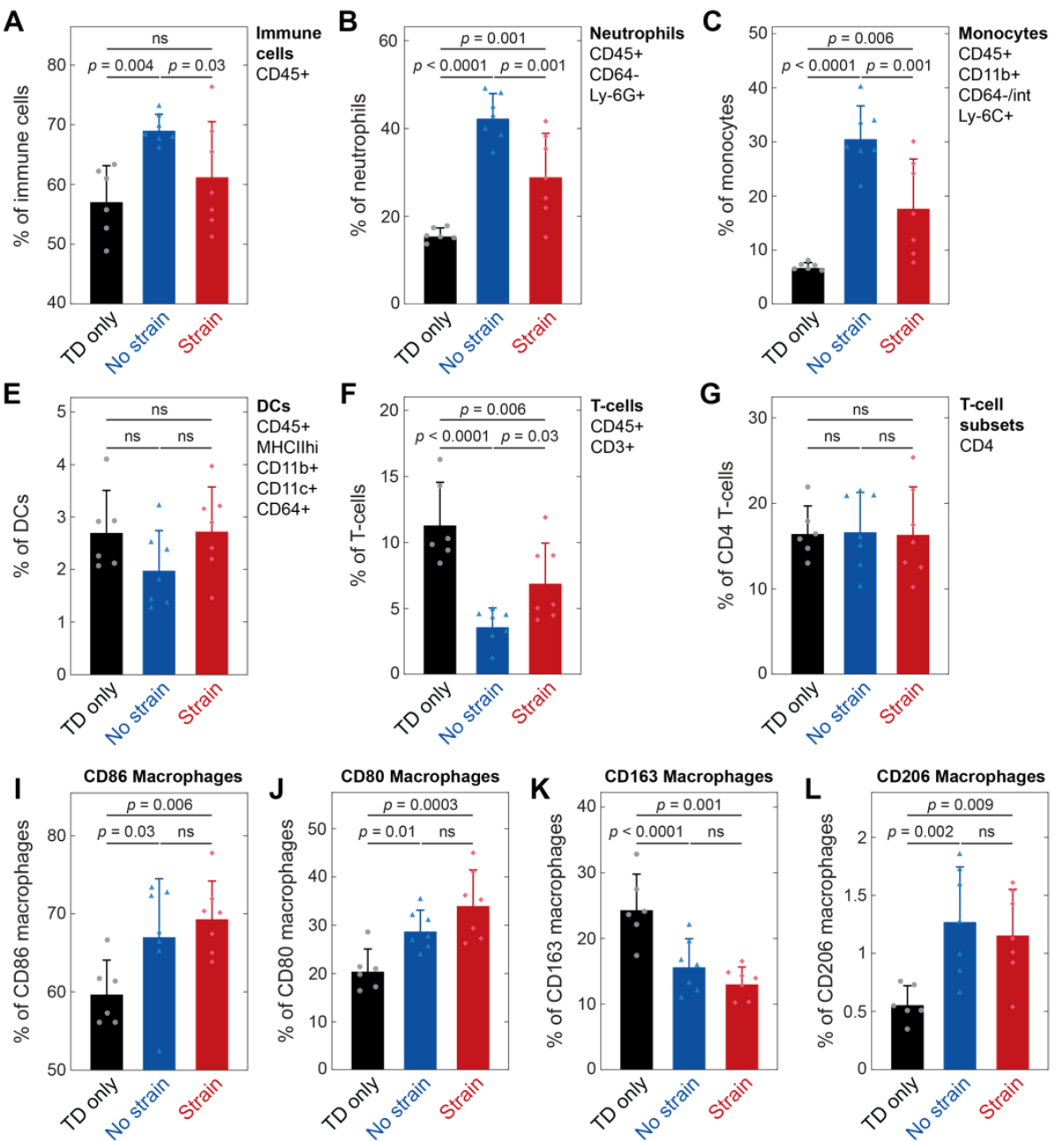

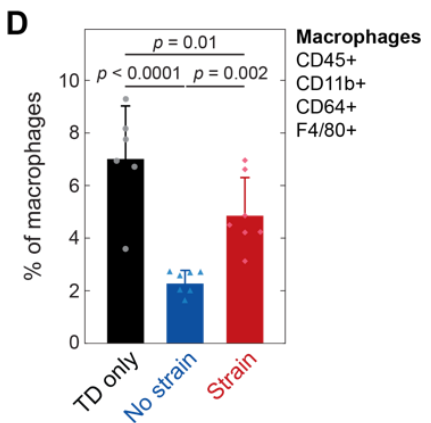

H
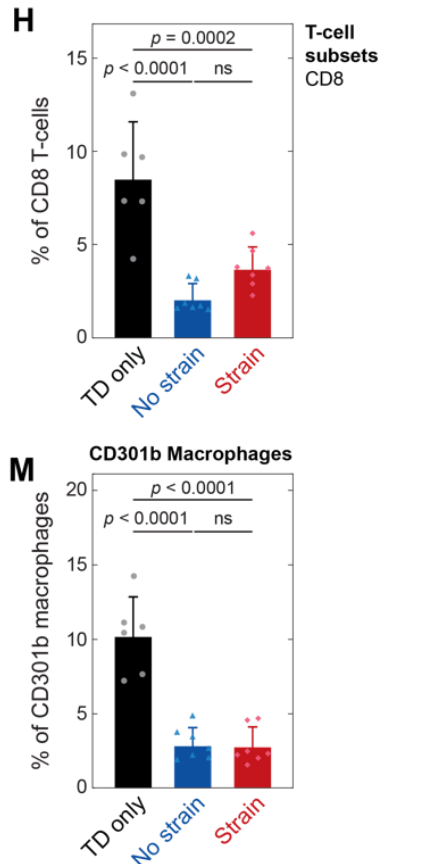

Fig. S20. Flow cytometry quantification of major immune cell populations and macrophage polarized states in D5 wounds. (A-M) Single cell suspensions were generated from excised wound and peri-wound tissues and stained for the indicated cell surface proteins. Percentage of immune cells (A), neutrophils (B), monocytes (C), macrophages (D), dendritic cells (DCs) (E), and T-cells $(\mathrm{F})$ and $\mathrm{T}$-cell subsets $(\mathrm{G}$ and $\mathrm{H})$. Percentage of macrophages expressing markers CD86 (I), CD80 (J), CD163 (K), CD206 (L), and CD301b (M). Each data point represents pooled cells from two mice (four wounds). Values represent the means $\pm \mathrm{SD}(n=6-7) . P$ values were derived from one way ANOVA with Fisher's LSD post-hoc tests. ns, not significant. 
A

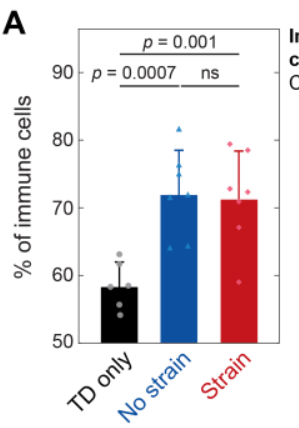

E

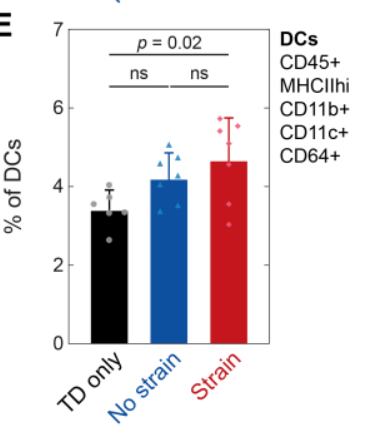

B

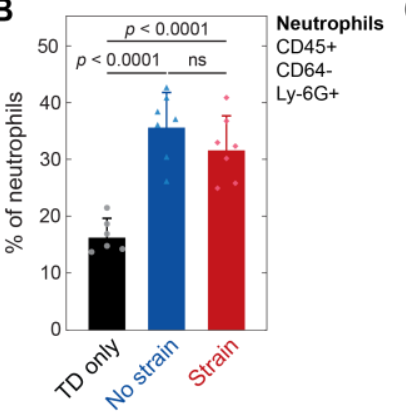

F
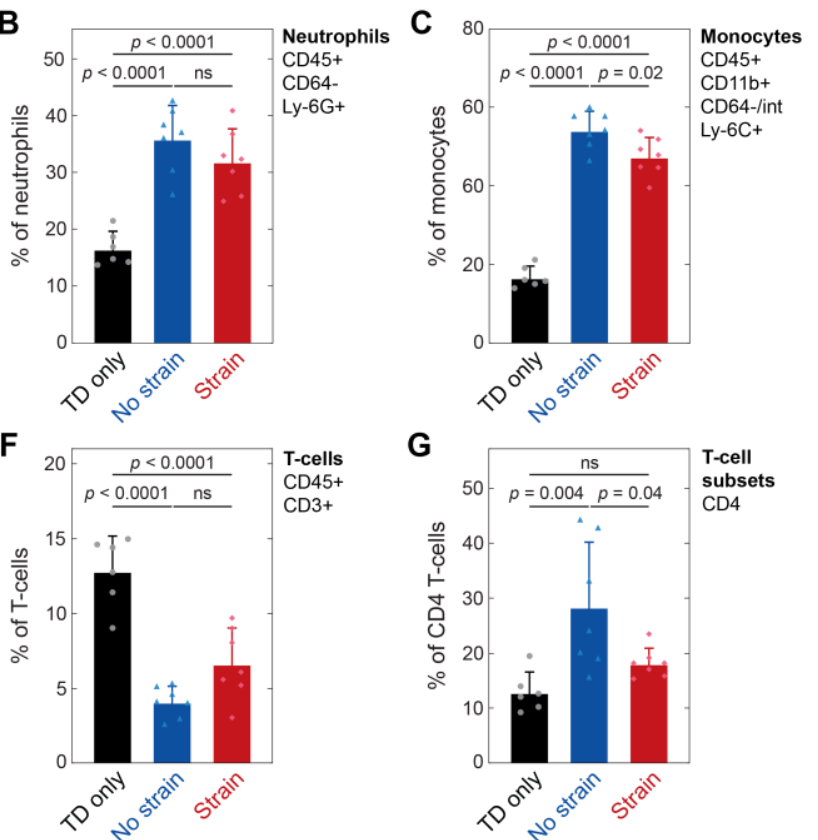
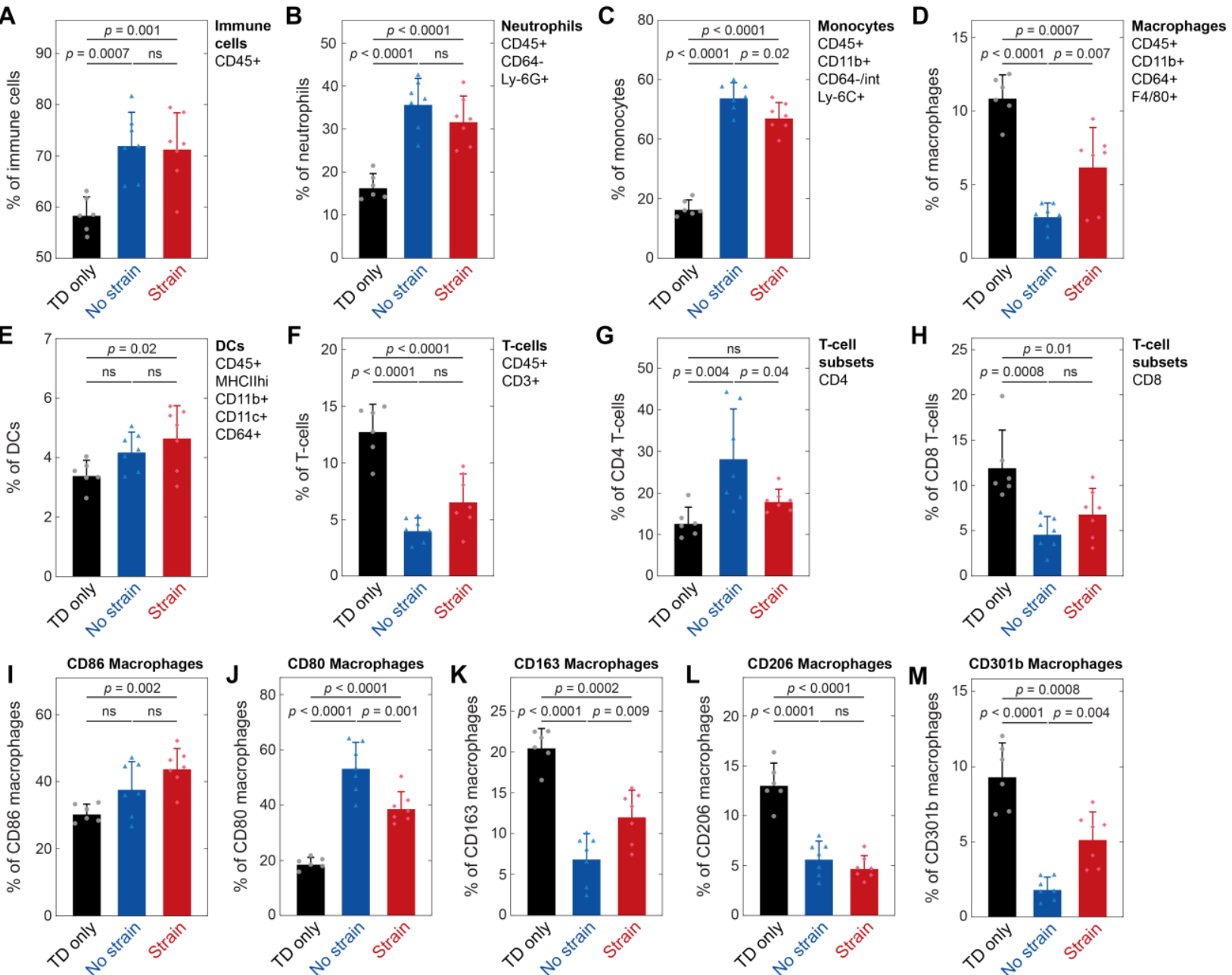

Fig. S21. Flow cytometry quantification of major immune cell populations and macrophage polarized states in D10 wounds. (A-M) Single cell suspensions were generated from excised wound and peri-wound tissues and stained for the indicated cell surface proteins. Percentage of immune cells (A), neutrophils (B), monocytes (C), macrophages (D), dendritic cells (DCs) (E), and T-cells $(\mathrm{F})$ and $\mathrm{T}$-cell subsets $(\mathrm{G}$ and $\mathrm{H})$. Percentage of macrophages expressing markers CD86 (I), CD80 (J), CD163 (K), CD206 (L), and CD301b (M). Each data point represents pooled cells from two mice (four wounds). Values represent the means \pm SD $(n=6-7) . P$ values were derived from one way ANOVA with Fisher's LSD post-hoc tests. ns, not significant. 
A

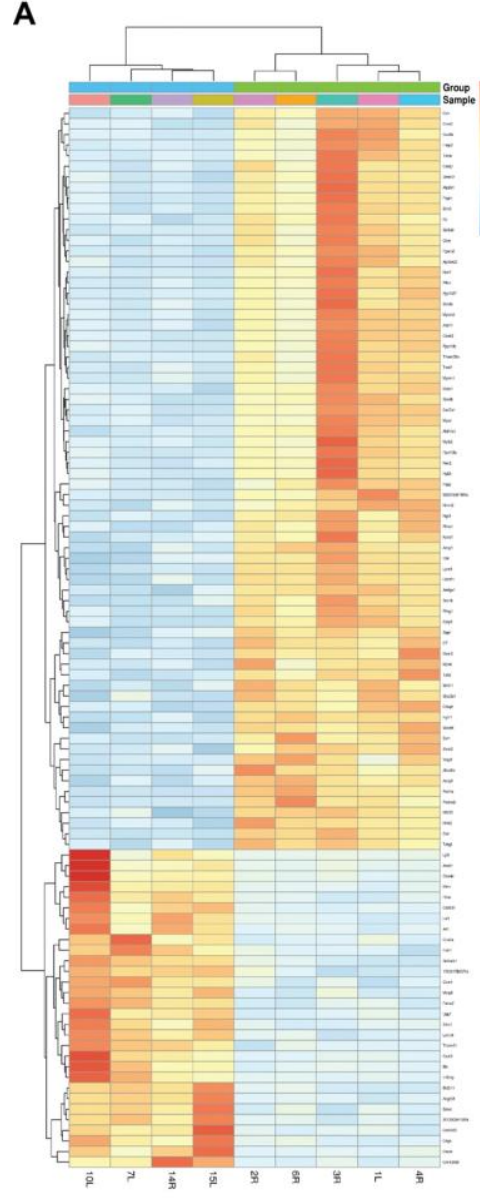

B

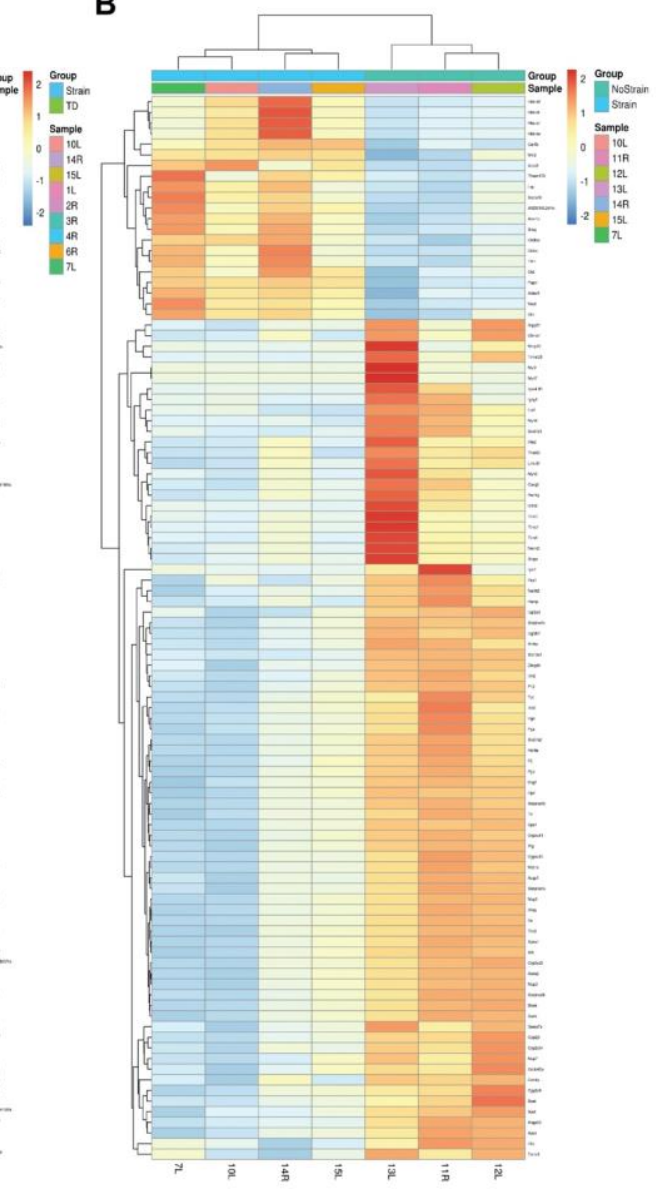

C

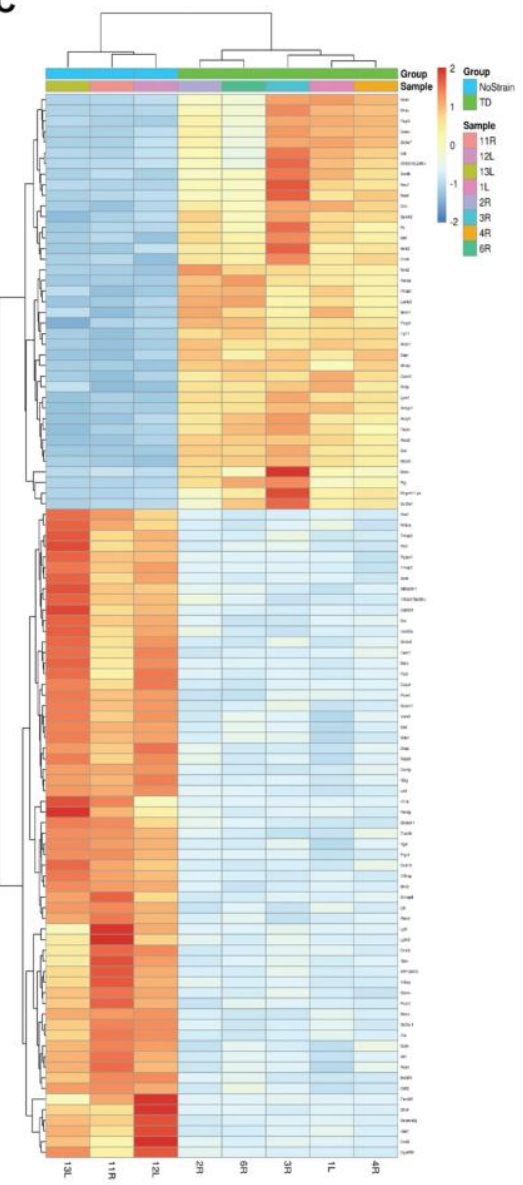

Fig. S22. Visualization of RNA sequencing results. (A-C) Heatmaps generated from the top 100 differentially expressed features of Strain vs TD (A), No strain vs Strain (B), and No strain vs TD (C) comparisons. Dendrograms were drawn from Ward hierarchical clustering. Higher expression levels correspond to warmer colors. 


\section{A}

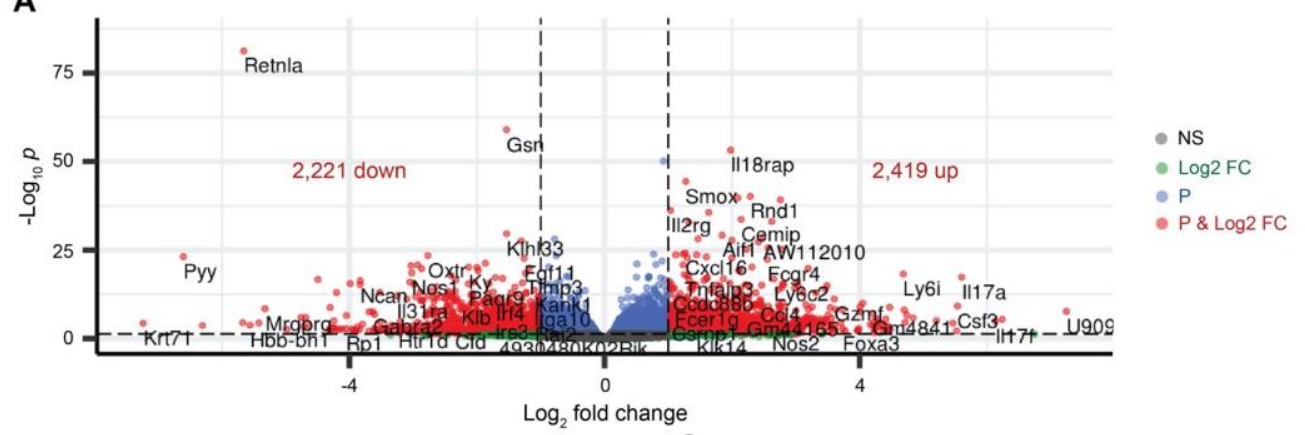

B
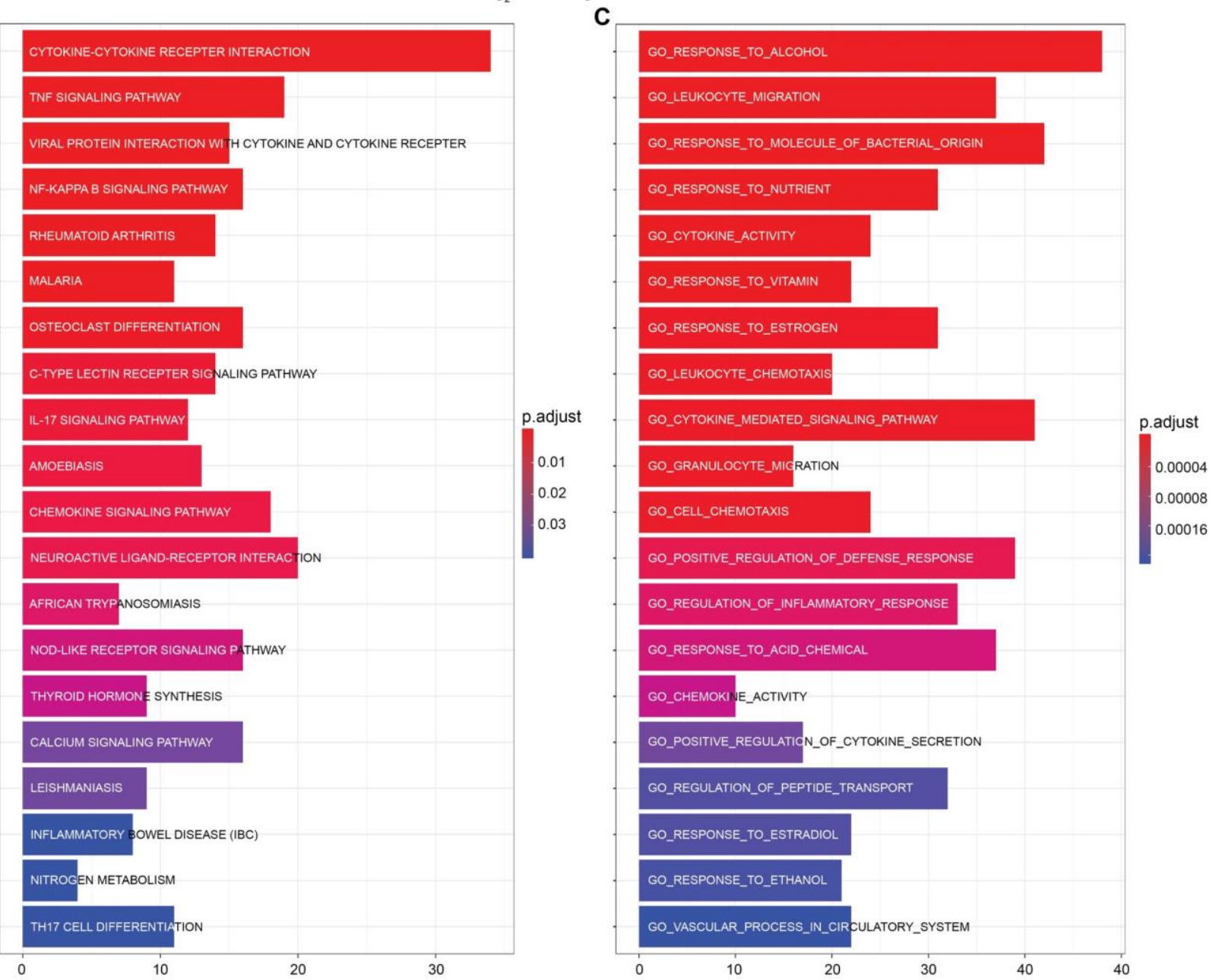

Fig. S23. No Strain vs TD differential expression and functional analyses. (A) Volcano plot displaying gene expression profile. Red colored data points represent genes that meet the thresholds of fold change (FC) above 1 or under -1 , False Discovery Rate (FDR) $<0.05$. (B and C) Functional over-representation analysis utilizing the top 500 differentially expressed genes results in gene ontology (GO) (B) and Reactome (C) databases. The $\mathrm{x}$-axis corresponds to the number of genes implicated in each pathway and the color of the bars correlates with the adjusted $p$-values as shown in the legends. 


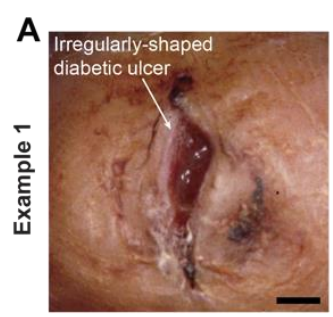

Diabetic foot ulcer

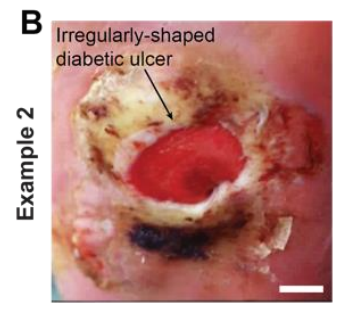

Diabetic foot ulcer

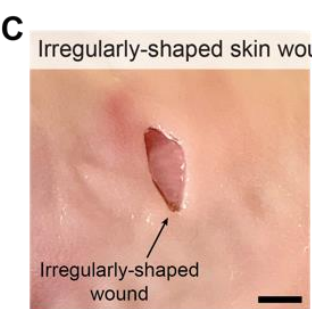

Ex vivo porcine skin

D

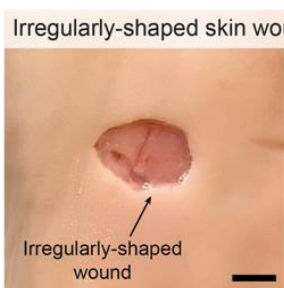

Ex vivo porcine skin

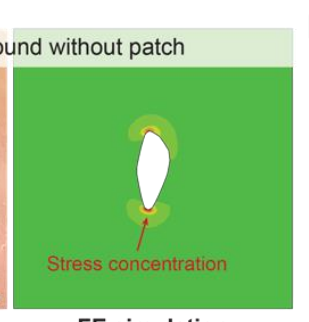

FE simulation

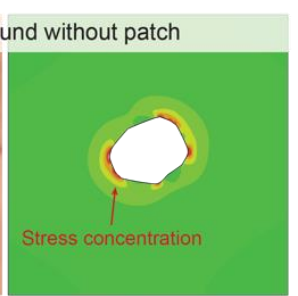

FE simulation

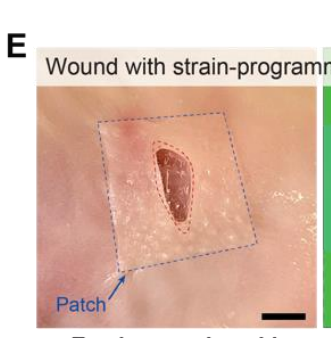

Ex vivo porcine skin

$\mathbf{F}$

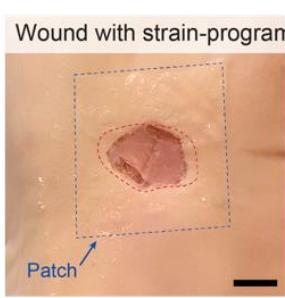

Ex vivo porcine skin

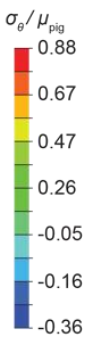

FE simulation

$$
\sigma_{\theta} / \mu_{\text {pig }}
$$

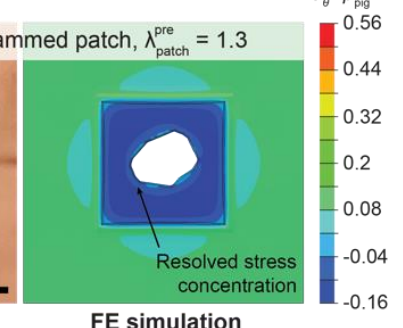

FE simulation

Fig. S24. Applicability of the strain-programmable patch to irregularly-shaped wounds. (A and B) Examples of irregularly-shaped diabetic foot ulcer (DFU) in patients. (C and D) Ex vivo porcine skin wounds and finite-element (FE) model results based on the example DFU. (E and F) Mechanical modulation of ex vivo porcine skin wounds and the corresponding FE model results by the strain-programmed patch $\left(\lambda_{\text {patch }}^{\text {pre }}=1.3\right)$. Wound contraction and removal of wound-edge stress concentration are indicated in the experimental images and FE results. The shear modulus of the porcine skin is denoted as $\mu_{\text {pig }}$ and the hoop stress in the porcine skin as $\sigma_{\theta}$. Scale bars, 5 $\mathrm{mm}(\mathrm{A}-\mathrm{F})$. 
bioRxiv preprint doi: https://doi.org/10.1101/2021.06.07.447423; this version posted June 7, 2021. The copyright holder for this preprint (which was not certified by peer review) is the author/funder, who has granted bioRxiv a license to display the preprint in perpetuity. It is made available under aCC-BY-NC-ND 4.0 International license.

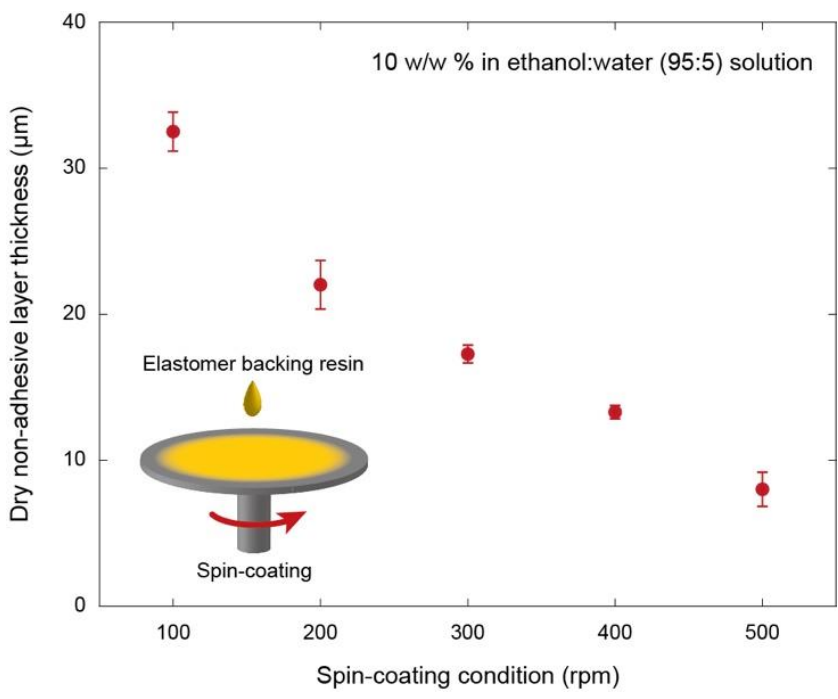

Fig. S25. Spin-coating of backing layer. Values represent the means $\pm \operatorname{SD}(n=4)$. 
bioRxiv preprint doi: https://doi.org/10.1101/2021.06.07.447423; this version posted June 7, 2021. The copyright holder for this preprint (which was not certified by peer review) is the author/funder, who has granted bioRxiv a license to display the preprint in perpetuity. It is made available under aCC-BY-NC-ND 4.0 International license.

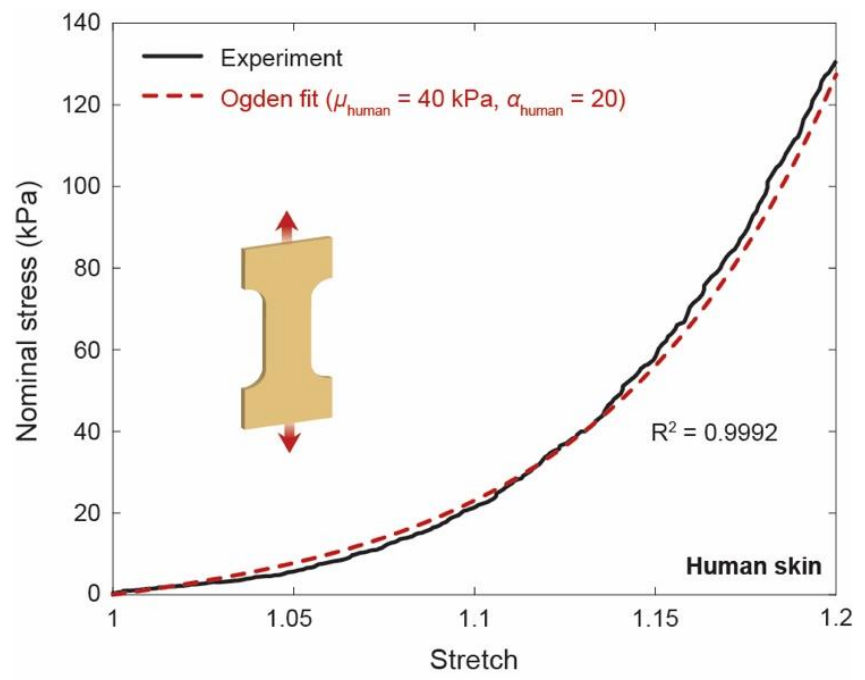

Fig. S26. Tensile properties of ex vivo human skin. Nominal stress vs. stretch curve for an ex vivo human skin fitted with the incompressible Ogden hyperelastic model. 


\section{Captions for other supplementary materials}

Data file S1. Complete lists of RNA-seq data.

Data file S2. Complete list of the antibody cocktail for flow cytometry.

Movie S1. Rapid adhesion and closure of an ex vivo porcine skin wound by an isotropically strainprogrammed patch.

Movie S2. Rapid adhesion and closure of an ex vivo porcine skin wound by an anisotropically strain-programmed patch.

Movie S3. On-demand removal of an adhered patch on an ex vivo porcine skin wound by applying a triggering solution.

Movie S4. Rapid adhesion and closure of wound in an ex vivo diabetic mouse skin by a strainprogrammable patch. 